\title{
LLNL-TR-630332
}

XML Network Description Language (XNDL) for ns-3

\section{ns-3 XSD v0.9}

L. E. Banks, P.D. Barnes, Jr., D.R.

Jefferson, S. Nikolaev

February 2013

Technical Report

submitted to the

United States Army Research Lab (ARL) 


\section{Disclaimer}

This document was prepared as an account of work sponsored by an agency of the United States government. Neither the United States government nor Lawrence Livermore National Security, LLC, nor any of their employees makes any warranty, expressed or implied, or assumes any legal liability or responsibility for the accuracy, completeness, or usefulness of any information, apparatus, product, or process disclosed, or represents that its use would not infringe privately owned rights.

Reference herein to any specific commercial product, process, or service by trade name, trademark, manufacturer, or otherwise does not necessarily constitute or imply its endorsement, recommendation, or favoring by the United States government or Lawrence Livermore National Security, LLC. The views and opinions of authors expressed herein do not necessarily state or reflect those of the United States government or Lawrence Livermore National Security, LLC, and shall not be used for advertising or product endorsement purposes.

This work performed under the auspices of the U.S. Department of Energy by Lawrence Livermore National Laboratory under Contract DE-AC52-07NA27344. 


\section{Table of Contents}

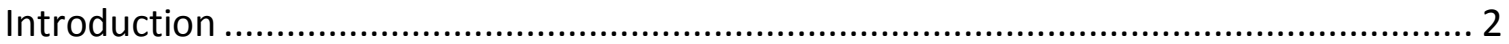

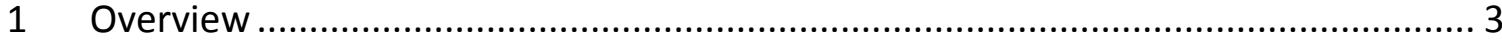

2 Document Conventions and History ................................................................... 3

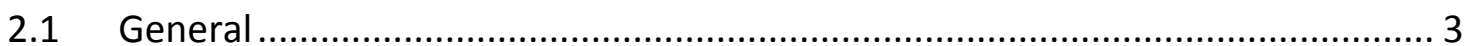

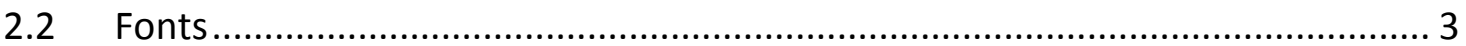

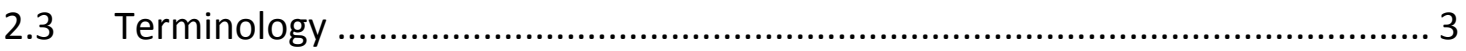

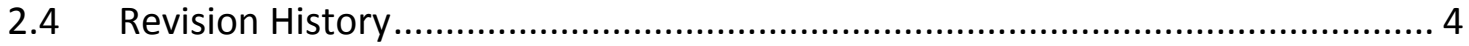

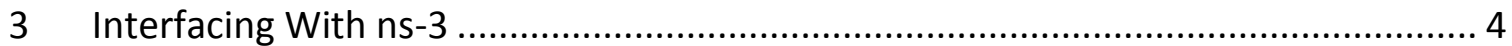

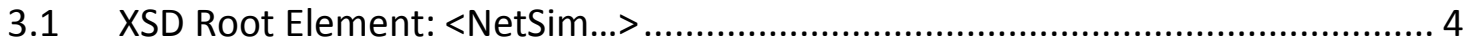

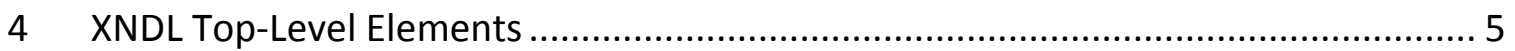

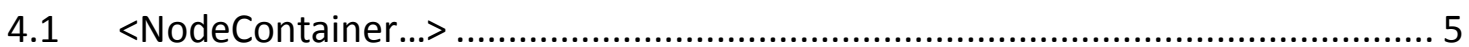

$4.2<$ Subnet...>

$4.3<$ Application...> and <ApplicationSet...> .................................................... 6

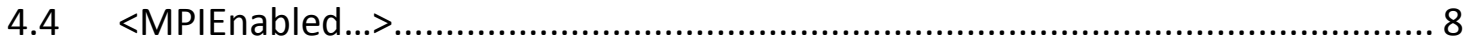

5 Support for High Performance Computing and MPI ............................................... 8

6 Ongoing and Future XNDL Development.......................................................... 10

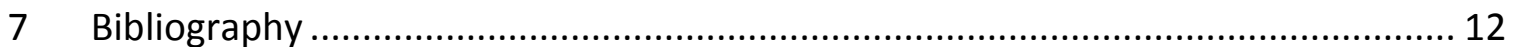

Appendix A. XNDL for ns-3 v0.9 - XSD Reference ........................................... A-1 


\section{Introduction}

This document is the "XML Network Description Language (XNDL) for ns-3 ns-3 XSD v0.9" addressing the Statement of Work for the Proposed Research in Network Simulation under contract L145271. This work performed by Lawrence Livermore National Laboratory (LLNL) is specifically for the Army Research Laboratory.

There exists a dichotomy between a desire to create generic network models that are framework-agnostic and models that are tailored to specific simulation implementations. Both methods have advantages and disadvantages. A generic approach would yield models that can be used and shared among many implementations and thus potentially gain support of a larger user community. However, there would remain a gap between concepts in a generic model and the corresponding representation needed for specific implementations. An approach that targets a specific implementation allows models to take full advantage of tighter integration and have a more explicit representation of simulation concepts and relationships. But locking into a specific implementation removes the portability aspect of the model. See Barnes, et al, for a more complete discussion [1].

XNDL is a XML-based architecture being developed at LLNL for the purpose modeling networks at a high level of abstraction while using a hierarchical structure of targeted grammars to interface with a broader base of network simulation frameworks and their associated tools (see Figure 1).

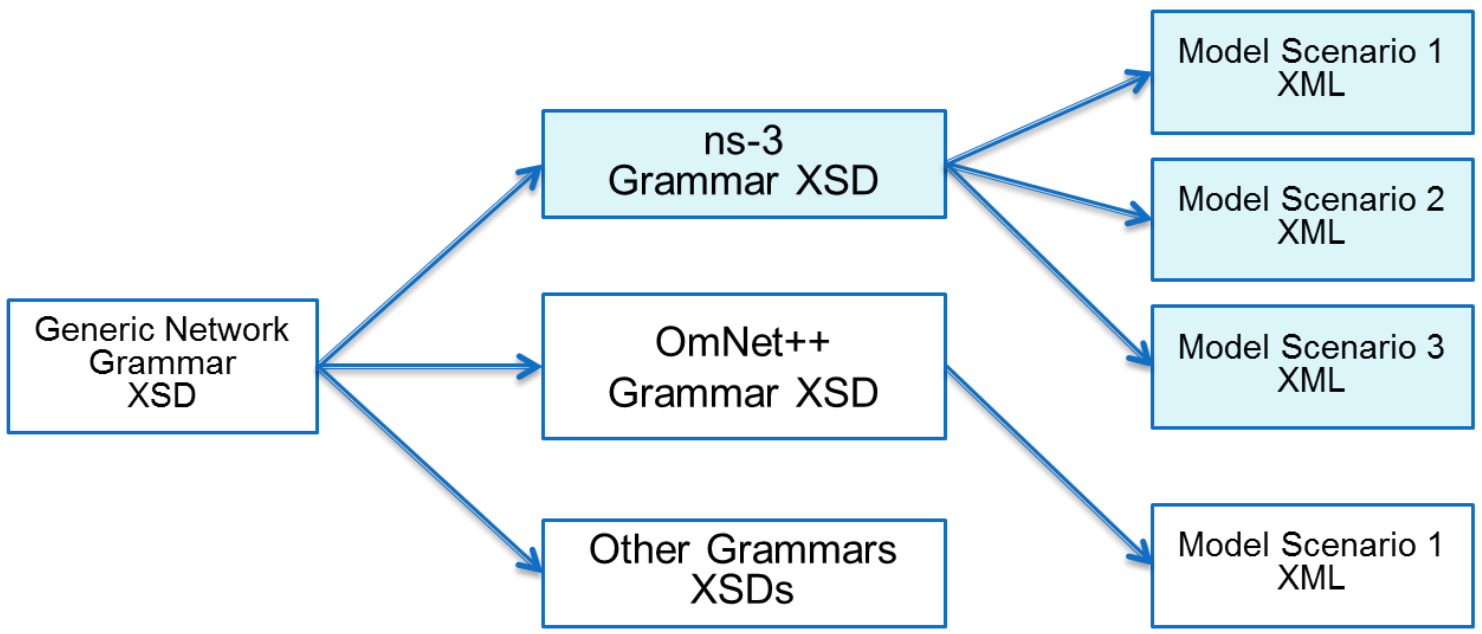

Figure 1: XNDL hierarchical architecture. XNDL for ns-3 is highlighted.

This document describes $X N D L$ for $n s-3$ including the grammar defined with a XML Schema Definition, or XSD, and the coupling between generated model scenarios and the ns-3 simulator. Supporting simulations on high performance computing clusters using MPI is also discussed. The full XSD for the ns-3 grammar is included in Appendix A. 


\section{Overview}

When working with a simulation tool there is often a desire to abstract the model from the simulation. This abstraction enables a modeler to focus on the objects, behaviors, and relationships being modeled, independently of the underlying implementation. A model definition can be retained and versioned, enabling repeatable simulations and tightly controlled studies by varying specific parameters in the model. If differing implementations of the simulation exist, an abstracted model can be more easily ported or mapped from one simulation framework to another.

The ns-3 Network Simulator lends itself nicely to this idea of creating a network model that is only loosely coupled to the actual implementation. Out of the box, ns-3 incorporates user defined scripts, which are actually $\mathrm{C}++$ or Python programs, to instantiate the objects with the desired parameterization. It is straightforward to have a parameterized model that can be parsed and interpreted to instantiate the corresponding objects in the simulation, by using the same mechanism as the ns-3 scripts.

XML has been chosen as the language to represent XNDL. XML is a popular format for expressing models with well-defined entities and relationships in an intuitive manner. An object in the real world can be represented by an XML element with a given set of attributes to capture the desired aspects of the object being modeled. Complexities of objects can be decomposed and represented by parent-child relationships in XML or composition of multiple XML elements. An XML Schema Definition, or XSD, specifies the grammar to which an XML representation must adhere.

\section{Document Conventions and History}

\subsection{General}

Notes are indicated by the clipboard icon to the left of text. These are used to make comments regarding planned future modifications and extensions.

\subsection{Fonts}

\begin{tabular}{||l|l||}
\hline \hline ITALICS & Used to emphasize a new concept, term, or definition \\
\hline BOLD & Used to emphasize a specific section of an XML code examples and snippets \\
\hline FIXED WIDTH & Used for all XML code examples and tags. \\
\hline \hline
\end{tabular}

\subsection{Terminology}

An XML tag will refer to either an opening tag for an element: <element>, or a closing tag: </element>. An XML element will refer to the opening and closing tags and all information contained within. 


\subsection{Revision History}

The current version of XNDL for ns-3 is 0.9. The following table will track the major modifications of this document by date in relation to the progressing versions of XNDL for ns-3.

Document Revisions

\begin{tabular}{||c|c|l|l||}
\hline \hline XNDL Version & Date & Contact & \multicolumn{1}{c||}{ Remarks } \\
\hline 0.9 & $2 / 22 / 2013$ & Eddy Banks & Initial version. \\
\hline & & & \\
\hline & & & \\
\hline & & & \\
\hline
\end{tabular}

\section{Interfacing With ns-3}

The current XSD allows specification of connected P2P and CSMA network topologies, and support for the configuration and distribution of several ns-3 applications. It also supports the configuration of packet capture (pcap), and trace file generation. The ns-3 simulator implementation has been augmented with a front-end module called: XMLSimulationReader that parses an input XML model and instantiates the ns-3 simulation objects using ns-3 helper classes in the same way as a traditional usercreated script.

\subsection{XSD Root Element: <NetSim...>}

The root element of the XSD model is $<$ NetSim $>$. The NetSim element contains attributes needed to initialize the simulation as well as containing the current schema version. The attributes are as follows:

- $\quad$ Name (required)

$\circ$ User-defined string name for this simulation model.

- P2pEnablePcapAll

O Boolean flag to turn on pcap format tracing for point-to-point devices.

- P2pEnableAsciitraceAll

O Boolean flag to turn on ascii tracing for point-to-point devices.

- CsmaEnablepcapall

- Boolean flag to turn on pcap format tracing for CSMA devices.

- CsmaEnableAsciitraceAll

- Boolean flag to turn on ascii tracing for CSMA devices.

- SchemaVersion (required)

- Current version of XNDL ns-3 model. 
Retaining the schema version is important in order to record what version of the model was used with a particular study. It is also needed as a connection point for the various tools in the XNDL framework. For example, various transformation tools, and the simulation implementation itself, may only be applicable to a specific version of the model and may not be applicable for older or newer versions.

The ns-3 network related elements are children of the $<$ NetSim $>$ root. These top-level elements are described next.

\section{XNDL Top-Level Elements}

\section{$4.1<$ NodeContainer...>}

The $<$ NodeContainer $>$ element in XNDL for ns-3 has a Name attribute and an optional Size attribute. If $\mathrm{Size}$ is given, then Size number of ns-3 nodes will be added to the model. The Name attribute is used to define a unique name allowing other elements to refer to a specific $<$ NodeContainer $>$. If the $<$ NodeContainer $>$ element does not specify the size attribute, then it is expected to contain <RefNode> and/or <ApplicationSet $>$ child elements. Each $<$ RefNode $>$ contains a name attribute referring to another $<$ NodeContainer $>$ containing the actual node, and an index attribute referring to the index of the node in the referenced $<$ NodeContainer $>$. For example, in Snippet 1 , the $<$ NodeContainer $>$ with Size attribute has Name="ALL_NODES". Other $<$ NodeContainer $>$ elements contain $<$ RefNode $>$ children that refer to the nodes that were created in the "ALL_NODES" $<$ NodeContainer $>$.

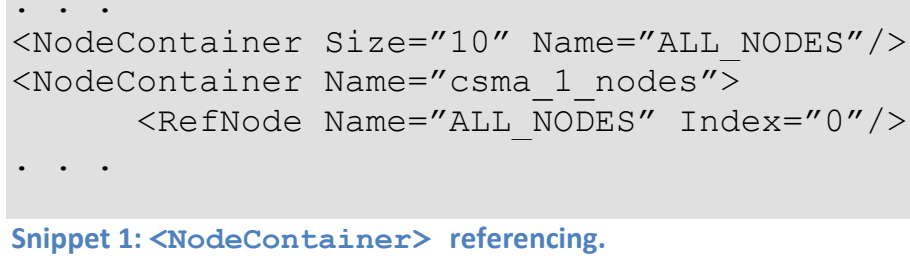

\section{2 <Subnet...>}

The $<$ Subnet $>$ elements contain attributes needed to parameterize the network linkage between nodes. As seen in Snippet 2, the $<$ NodeContainer $>$ is referenced by Name and again the actual nodes are linked via the Index attribute on a $<$ RefNode $>$ child element. 


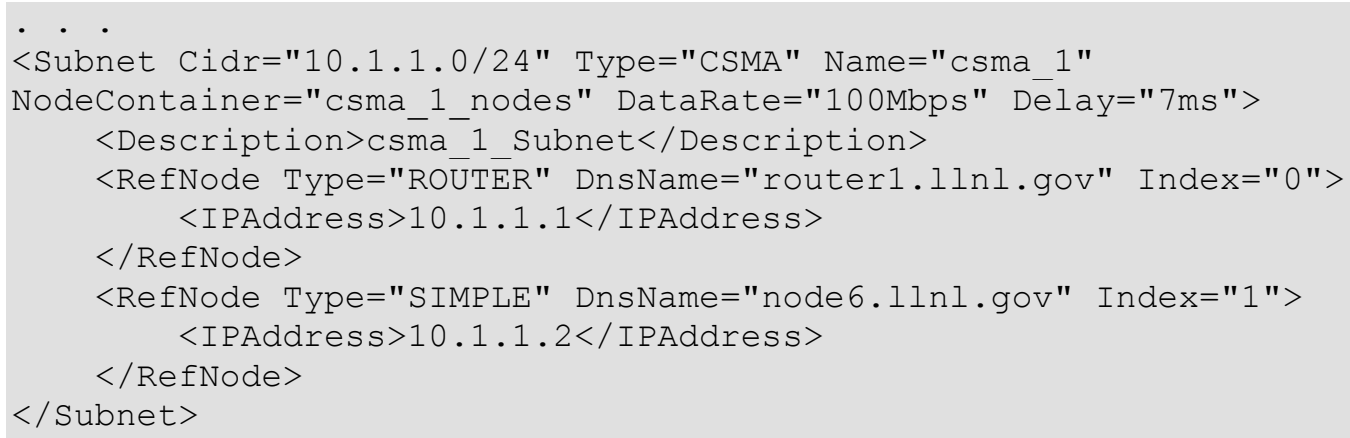

Snippet 2: <Subnet> element

In this case, the Index attribute of the $<$ RefNode $>$ element refers implicitly to the $<$ NodeContainer $>$ attribute of the containing < Subnet $>$ element. As can be seen, the $<$ Subnet $>$ element contains enough information to invoke several ns-3 helper classes.

\section{3 <Application...> and <ApplicationSet...>}

XNDL for ns-3 contains models for several of the ns-3 example applications including:

- bulkSend / PacketSink

- csmaPing

- genericApp

- onOffApp

- udpClientServer

- udpEcho

The <Application> element contains a user-defined Name attribute as well as any attributes specific to the application. The $<$ Applicationset $>$ element contains $<$ Application $>$ s and other $<$ ApplicationSets $>$ incorporated by reference to their Name attributes. In turn, a $<$ NodeContainer $>$ can refer to $<$ Application $>$ s and $<$ ApplicationSets $>$ by reference to the same Name, which has the effect of instantiating the referenced applications on nodes in the $<$ NodeContainer $>$. As seen in Snippet 3, the $<$ NodeContainer $>$ installs two different $<$ ApplicationSets $>$ on specific nodes. 


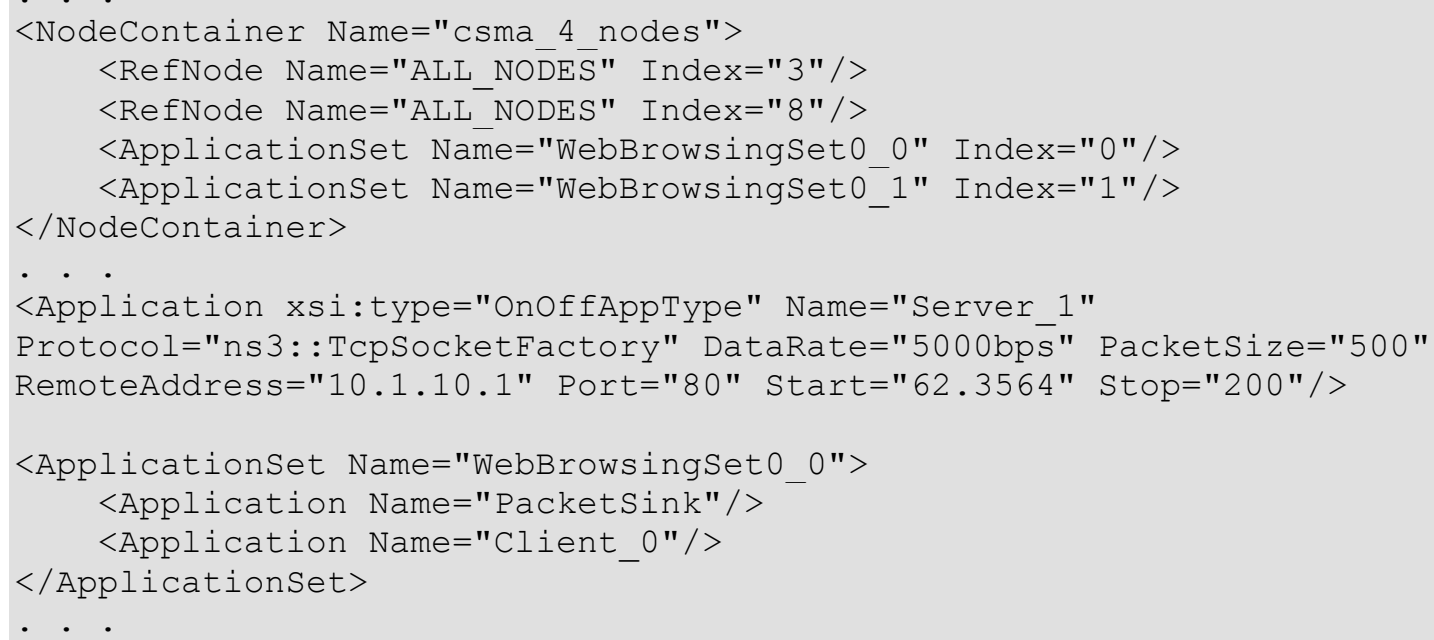

Snippet 3: <Application> and <ApplicationSet> elements.

\subsubsection{Generic Application Element}

XNDL provides support for applications which are not yet specified in the XSD. A generic application type, modeled with xsi: type of GenericAppType, has attributes for Name, Start, Stop and Type - but also contains <Attribute> child elements each of which contain Name and Value pair attributes to specify parameterization of the application. The XSD diagram for <GenericAppType> is shown in Figure 2.

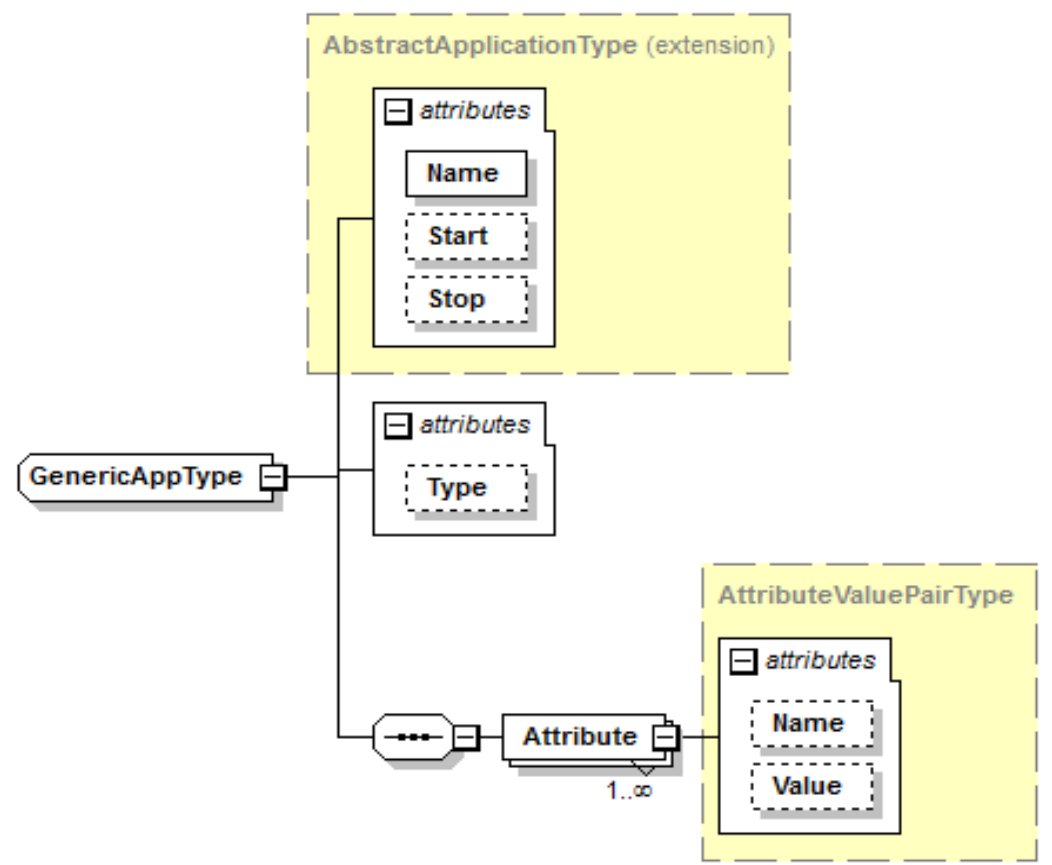

Generated by XMLSpy

www.altova.com

Figure 2: GenericAppType diagram. 
An example of defining a generic application with similar parameters as the OnOffAppType shown in Snippet 3 is shown in Snippet 4.

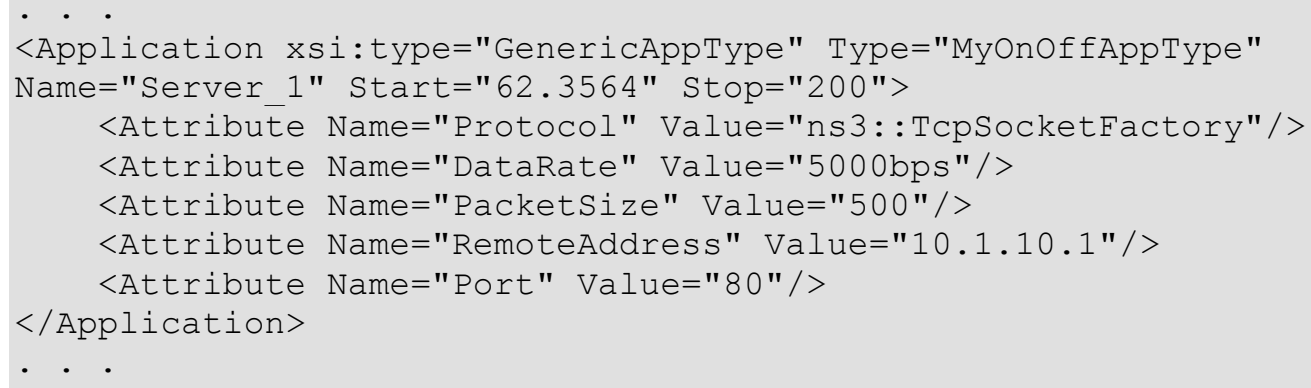

Snippet 4: Defining a generic application.

As with the other application types, the parent $<$ NodeContainer $>$ specifies the Nodes on which the application will run via $<$ RefNode $>$ elements .

\section{$4.4<$ MPIEnabled...>}

This is a simple element that currently serves as a flag to indicate if the simulation is to be a parallel implementation running on a high performance computing cluster using message passing interface (MPI). More detailed discussion follows in section 5: Support for High Performance Computing and MPI.

Since this element is specific to the model as a whole it will be moved to an attribute of the <NetSim $>$ root node in the next release of XNDL for ns-3.

\section{Support for High Performance Computing and MPI}

One of the goals at LLNL is to parallelize the network simulation using Message Passing Interface, MPI. To support this in the Model, the XSD includes an extra XML element: $<$ MPIEnabled $>1</$ MPIEnabled $>$ to indicate a MPI job. The parameter value of 0 indicates a non-MPI simulation; any non-zero value indicates a parallel network simulation. This element is a child of the $<$ Nets im $>$ element as shown in Snippet 5.

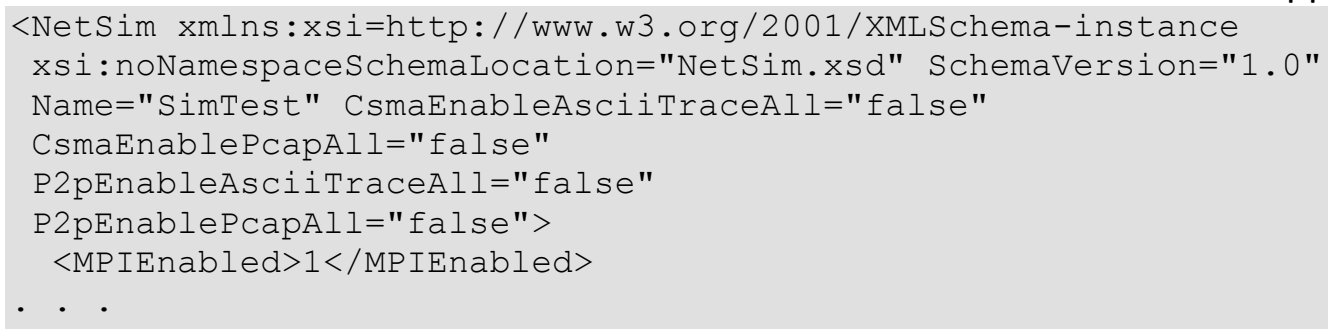

Snippet 5: Element to indicate MPI run. 
In preparing a simulation input for a parallel run, the network topology must be partitioned and assigned to ranks for processing on the computing cluster. The process flow is shown in Figure 3.

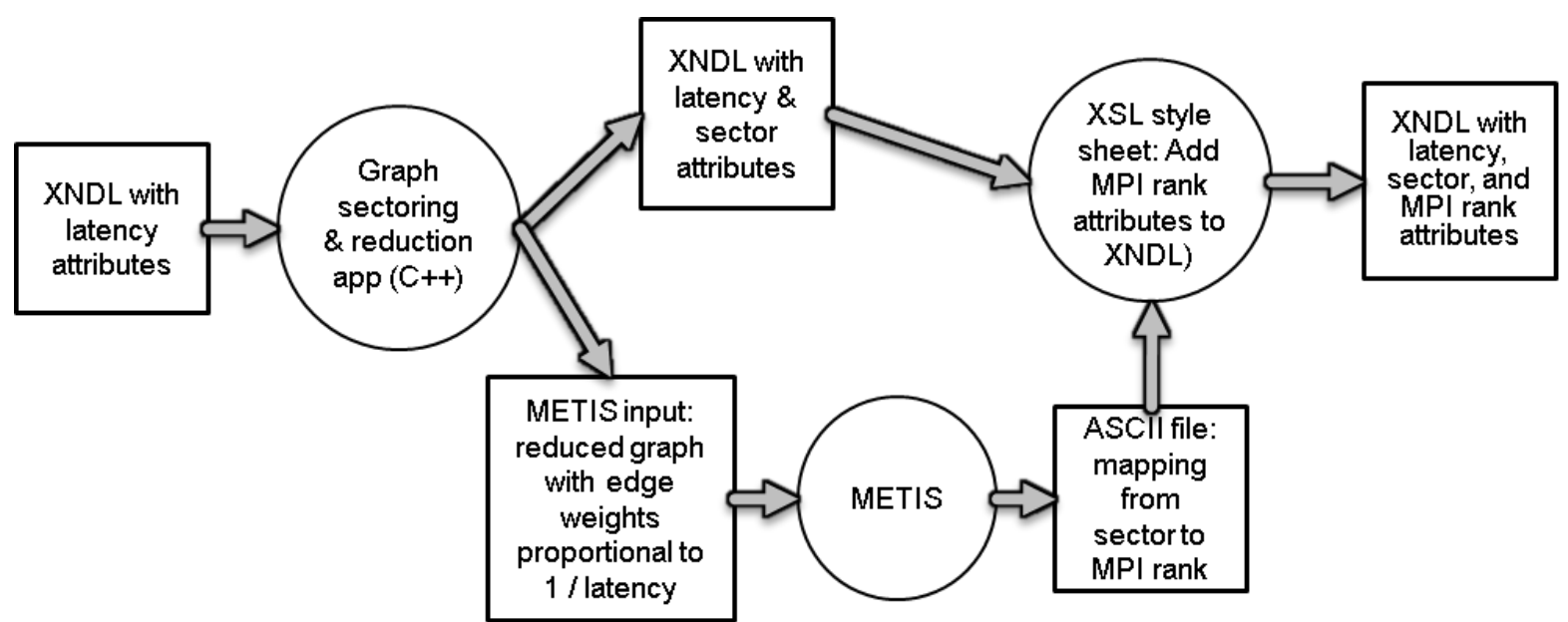

Figure 3: Automatic partitioning process flow.

The network model is first annotated for partitioning by the use of a Sector attribute on the $<$ NodeContainer $>$ and $<$ RefNode $>$ elements. All nodes with the same sector value must end up on the same MPI rank, because the underlying channel cannot be partitioned. For example, as shown in Snippet 6, a $<$ NodeContainer $>$ whose nodes are all on a single CSMA channel should remain in the same partition, thus the $<$ NodeContainer $>$ is assigned a Sector attribute for that CSMA channel. A $<$ NodeContainer $>$ referencing nodes on a point-to-point channel may be partitioned, hence the $<$ RefNode $>$ s can have different sector values.

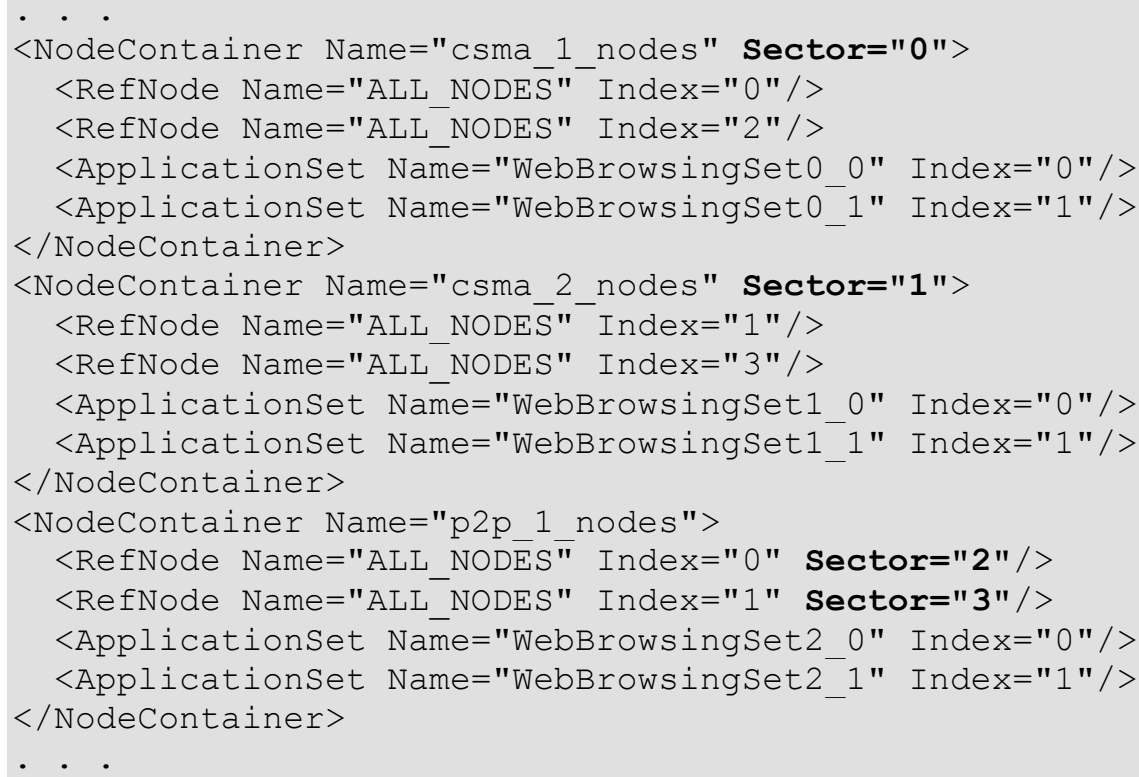

Snippet 6: Sector labeling based on latency attributes and Node types. 
A stand-alone sector labeling tool assigns sector labels where needed, and outputs the sector graph in METIS format. The METIS graph partitioning tool is used to assign sectors to MPI ranks based on its partitioning logic. An XSLT transform then applies the Sectorto-rank assignments reported by METIS to the original XML, by adding the systemId attribute to the $<$ NodeContainers $>$ and/or $<$ RefNode $>$ s, as shown in Snippet 7. The example in Snippet 6 and Snippet 7 illustrate the possibility of more than one sector being processed on the same rank.

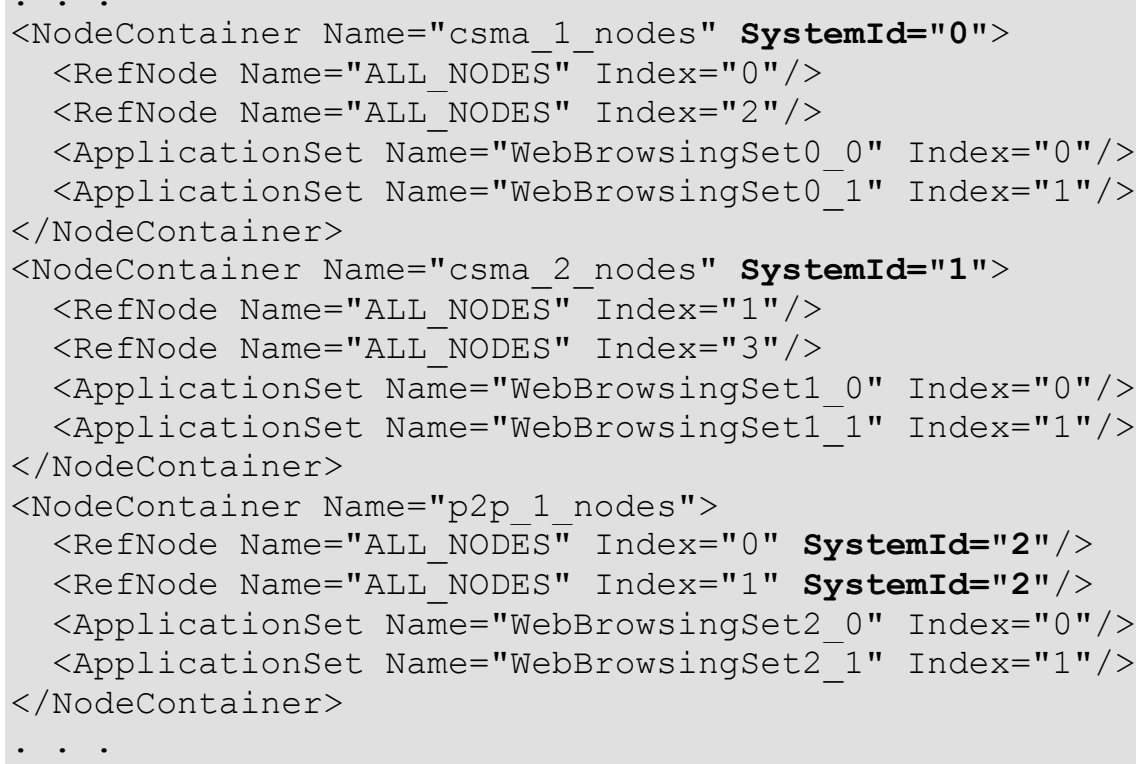

Snippet 7: Sys temId attribute for rank assignment based on METIS partitioning.

This three-step process, Sector labeling, partitioning, then application of SystemIds to Sectors, facilitates reuse of the Sector labeling, which need be performed only once for a given model. The Sector-labeled XML description can be reused for different partitioning strategies, for different numbers of MPI ranks, or different partitioning functions.

\section{Ongoing and Future XNDL Development}

This version of the XNDL for ns-3 model is the initial version currently being used for specifying ns-3 input specifications with XML. This model will be expanded to support the ongoing studies of large-scale network simulations.

Some of the next steps for the development of the XNDL framework include:

- Condensing parameterization on elements to a more general representation enabling reuse within an XML input specification while minimizing repetition. 
- Continue expanding the ns-3 specific grammar to incorporate more ns-3 model types, particularly wireless channels.

- Create the connecting tools in the XNDL framework to enable automation of simulation pre-processing - such as the METIS partitioning.

- Create a higher level network grammar to allow construction of generic models that can then be mapped to the XNDL ns-3 grammar or a different simulation implementation (refer back to Figure 1).

As XNDL evolves, so will this document to reflect the growing capabilities of the entire framework. A full reference of the XSD, including diagrams and source, is contained in Appendix A. 


\section{Bibliography}

[1] P. D. Barnes, B. B. Abelev, L. E. Banks, J. M. Brase, D. R. Jefferson, S. Nikolaev, S. G. Smith and R. A. Soltz, "Integrating ns-3 Model Construction, Description, Preprocessing, Execution, and Visualization," 2013.

[2] ns-3 Network Simulator Tutorial; Release ns-3.15, ns-3 Project, 2012.

[3] ns-3 Network Simulator Manual, Release-3.15, ns-3 Project, 2012.

[4] ns-3 Model Library; Release ns-3.15, ns-3 Project, 2012. 
LLNL-TR-630332

\title{
Appendix A. XNDL for ns-3 v0.9 - XSD Reference
}

\author{
Schema NetSim.xsd

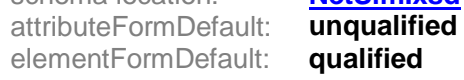

Complex types

BulkSendAppType

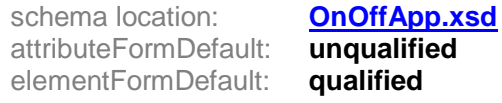


LLNL-TR-630332

$\begin{array}{ll}\text { attributeFormDefault: } & \text { unqualified } \\ \text { elementFormDefault: } & \text { qualified }\end{array}$

Complex types

UdpTraceClientAppType

schema location: $\quad$ V4PingApp.xsd

elementFormDefault: qualified

Complex types

V4PingType

$\begin{array}{ll}\text { schema location: } & \text { GenericApp.xsd } \\ \text { attributeFormDefault: } & \begin{array}{l}\text { unqualified } \\ \text { elementFormDefault: }\end{array} \\ \text { qualified }\end{array}$

Complex types

AttributeValuePairType

GenericAppType

$\begin{array}{ll}\text { schema location: } & \text { SimpleWebClientApp.xsd } \\ \text { attributeFormDefault: } & \text { unqualified }\end{array}$

elementFormDefault: qualified

Complex types

SimpleWebClientAppType

$\begin{array}{ll}\text { schema location: } & \text { SimpleWebServerApp.xsd } \\ \begin{array}{ll}\text { attributeFormDefault: } \\ \text { elementFormDefault: }\end{array} & \text { unqualified } \\ \text { qualified }\end{array}$

Complex types

SimpleWebServerAppType 
LLNL-TR-630332

element NetSim

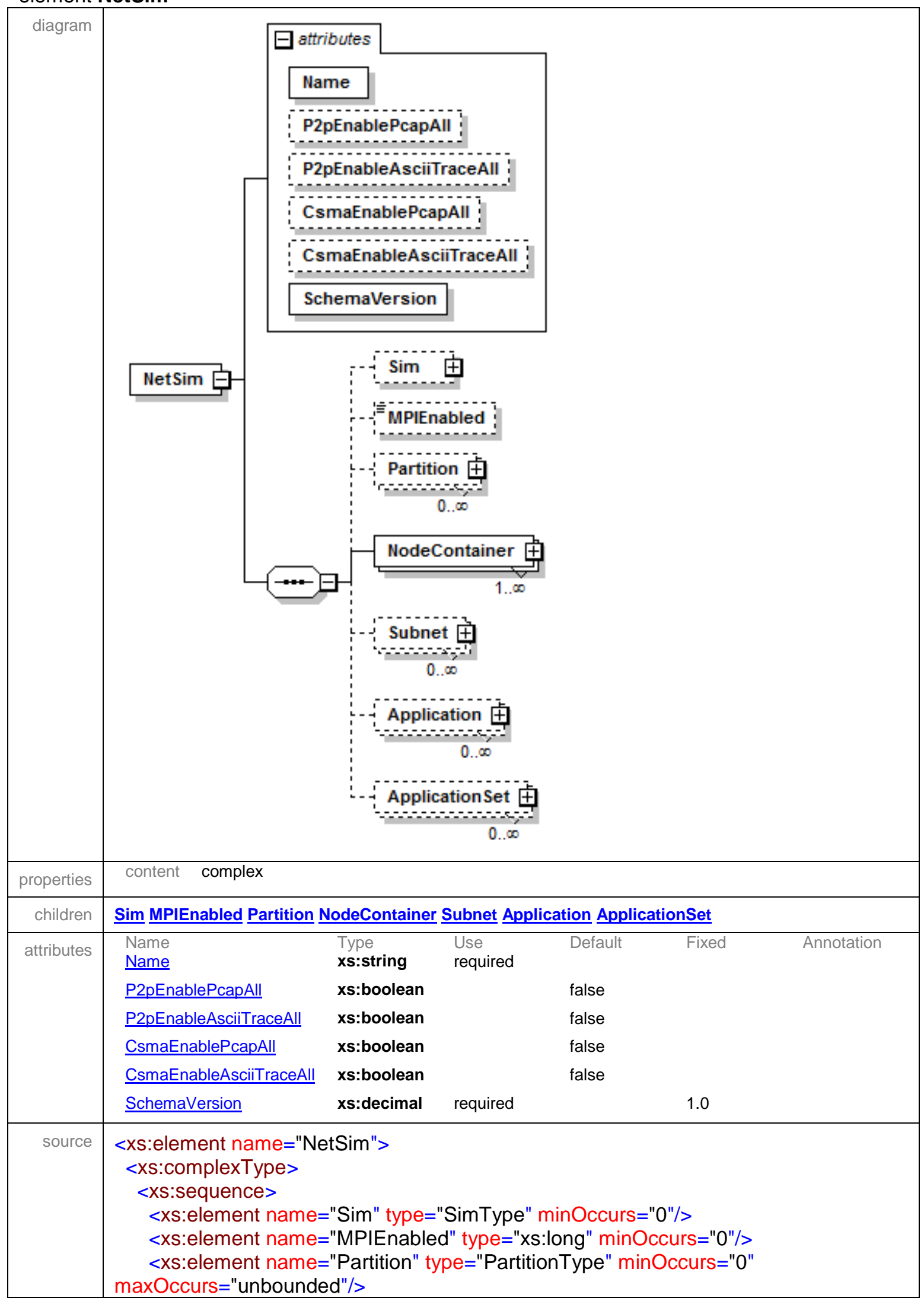




\begin{tabular}{|c|}
\hline $\begin{array}{l}\text { <!-- A NodeContainer element specifies a group nodes. --> } \\
\text { <xs:element name="NodeContainer" type="NodeContainerType" } \\
\text { maxOccurs="unbounded"/> } \\
\text { <!-- A Subnet element defines the Node/Interface pairings and throughput } \\
\text { characteristics for a subnet. --> } \\
\text { <!-- Each subnet is associated, via the NodeContainerName attribute, with a single } \\
\text { NodeContainer. --> } \\
\text { <!-- The set of all Subnet elements define the topology of the network. --> } \\
\text { <xs:element name="Subnet" type="SubnetType" minOccurs="0" } \\
\text { maxOccurs="unbounded"/> } \\
\text { <!-- The specification of an application --> } \\
\text { <xs:element name="Application" type="AbstractApplicationType" minOccurs="0" } \\
\text { maxOccurs="unbounded"/> } \\
\text { <!-- A set of applications that can be installed on one of more nodes --> } \\
\text { <xs:element name="ApplicationSet" type="ApplicationSetType" minOccurs="0" } \\
\text { maxOccurs="unbounded"/> } \\
\text { </xs:sequence> } \\
\text { <xs:attribute name="Name" type="xs:string" use="required"/> } \\
\text { <xs:attribute name="P2pEnablePcapAll" type="xs:boolean" default="false"/> } \\
\text { <xs:attribute name="P2pEnableAsciiTraceAll" type="xs:boolean" default="false"/> } \\
\text { <xs:attribute name="CsmaEnablePcapAll" type="xs:boolean" default="false"/> } \\
\text { <xs:attribute name="CsmaEnableAsciiTraceAll" type="xs:boolean" default="false"/> } \\
\text { <xs:attribute name="SchemaVersion" type="xs:decimal" use="required" } \\
\text { fixed="1.0"/> } \\
\text { </xs:complexType> } \\
\text { </xs:element> }\end{array}$ \\
\hline
\end{tabular}

attribute NetSim/@Name

\begin{tabular}{|r|l|}
\hline type & xs:string \\
\hline properties & use required \\
\hline source & $<x$ s:attribute name="Name" type="xs:string" use="required"/> \\
\hline
\end{tabular}

attribute NetSim/@P2pEnablePcapAll

\begin{tabular}{|r|l|}
\hline type & xs:boolean \\
\hline properties & default false \\
\hline source & <xs:attribute name="P2pEnablePcapAll" type="xs:boolean" default="false"/> \\
\hline
\end{tabular}

attribute NetSim/@P2pEnableAsciiTraceAll

\begin{tabular}{|r|l|}
\hline type & xs:boolean \\
\hline properties & default false \\
\hline source & $<x s: a t t r i b u t e ~ n a m e=" P 2 p E n a b l e A s c i i T r a c e A l l "$ type="xs:boolean" default="false"/> \\
\hline
\end{tabular}

attribute NetSim/@CsmaEnablePcapAll 
LLNL-TR-630332

\begin{tabular}{|c|c|}
\hline properties & default false \\
\hline source & <xs:attribute name="CsmaEnablePcapAll" type="xs:boolean" default="false"/> \\
\hline
\end{tabular}

attribute NetSim/@CsmaEnableAsciiTraceAll

\begin{tabular}{|c|c|}
\hline type & xs:boolean \\
\hline properties & default false \\
\hline source & <xs:attribute name="CsmaEnableAsciiTraceAll" type="xs:boolean" default="false"/> \\
\hline
\end{tabular}

attribute NetSim/@SchemaVersion

\begin{tabular}{|r|l|}
\hline type & xs:decimal \\
\hline properties & $\begin{array}{r}\text { use required } \\
\text { fixed } 1.0\end{array}$ \\
\hline source & $<x$ :attribute name="SchemaVersion" type="xs:decimal" use="required" fixed="1.0"/> \\
\hline
\end{tabular}

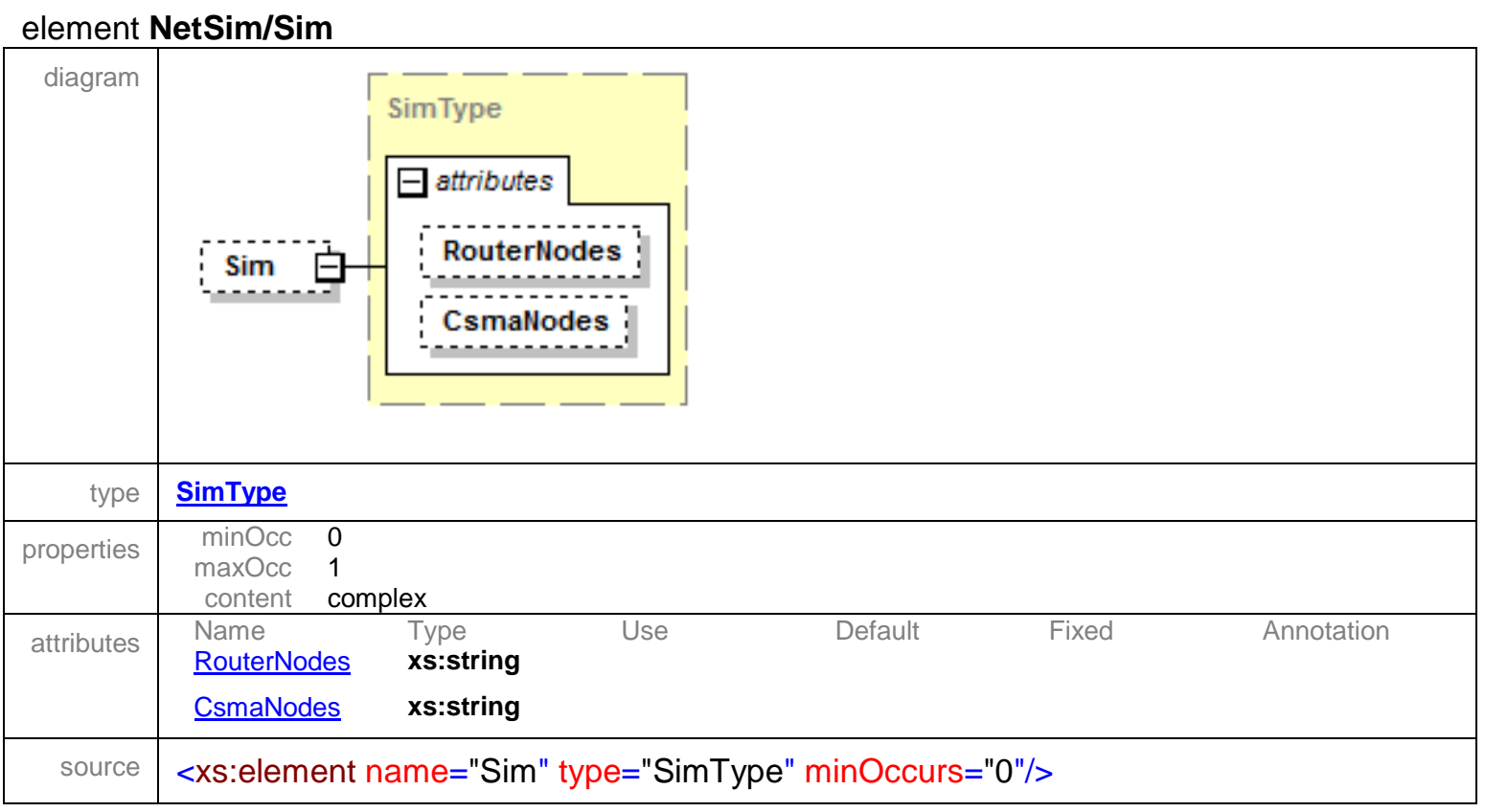

\section{element NetSim/MPIEnabled}

\begin{tabular}{|r|c|}
\hline diagram & \\
\hline type & xs:Iong \\
\hline properties & $\begin{array}{c}\text { minOcc } \\
\text { maxOcc } 1 \\
\text { content simple }\end{array}$ \\
\hline source & $<x$ :element name="MPIEnabled" type="xs:long" minOccurs="0"/> \\
\hline
\end{tabular}


element NetSim/Partition

\begin{tabular}{|r|l|l|}
\hline diagram & \\
&
\end{tabular} \mid

element NetSim/NodeContainer

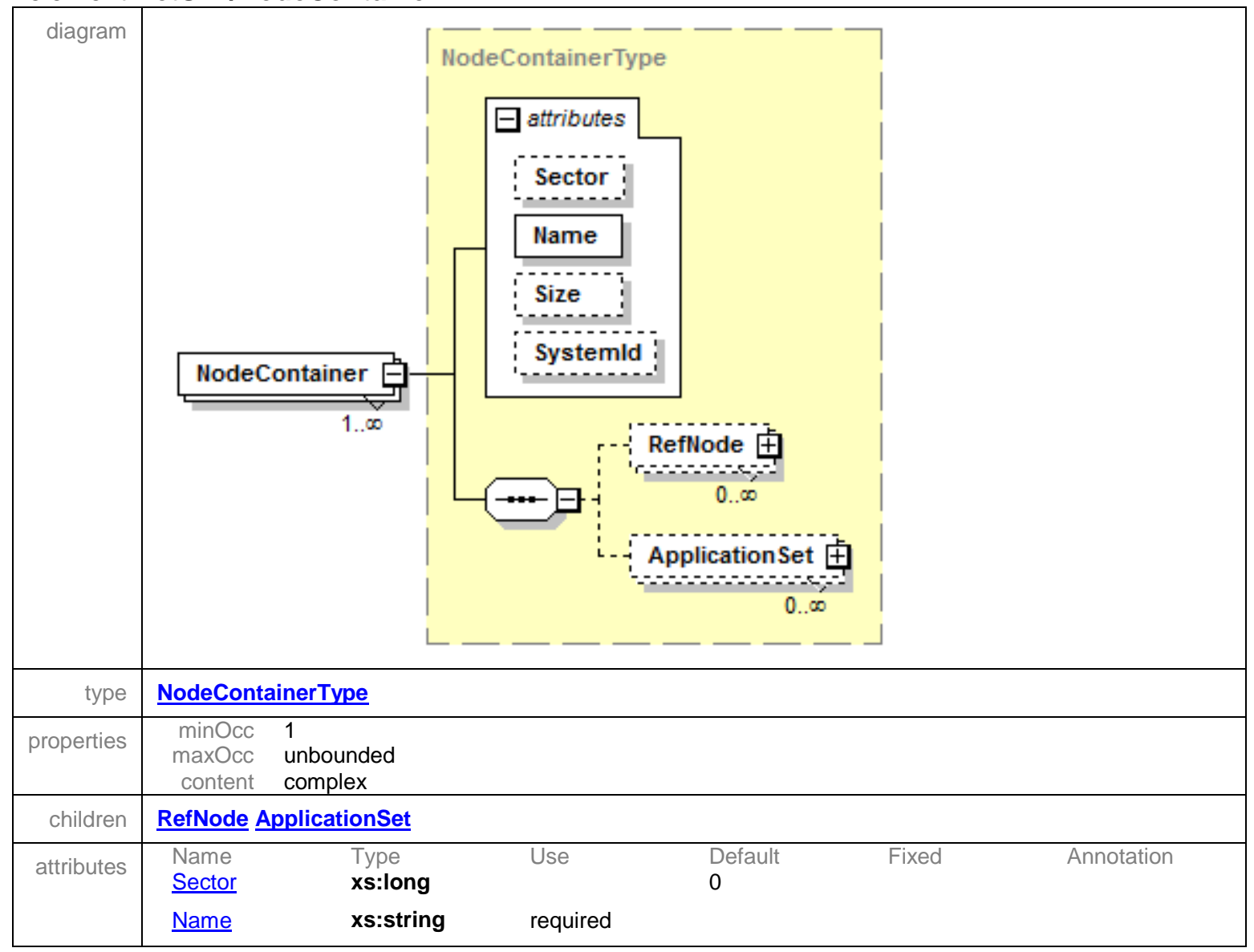


LLNL-TR-630332

\begin{tabular}{|c|c|c|c|}
\hline & $\underline{\text { Size }}$ & xs:Iong & 0 \\
\hline & Systemld & xs:Iong & 0 \\
\hline source & $\begin{array}{l}<x s: e l e m \\
\text { maxOccu }\end{array}$ & $\begin{array}{l}\text { ne="Nc } \\
\text { bounde }\end{array}$ & VodeContainerType" \\
\hline
\end{tabular}

element NetSim/Subnet

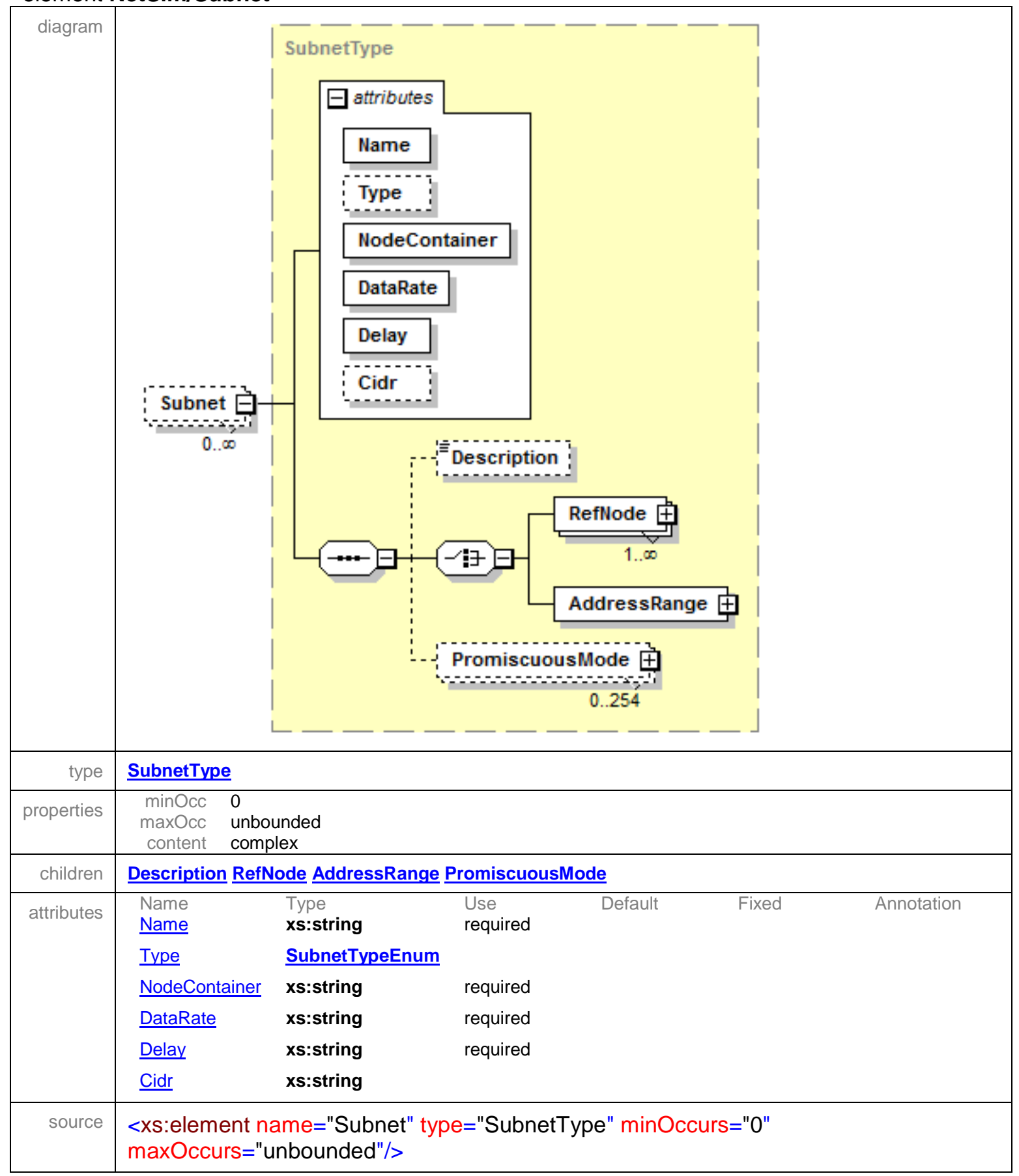


element NetSim/Application

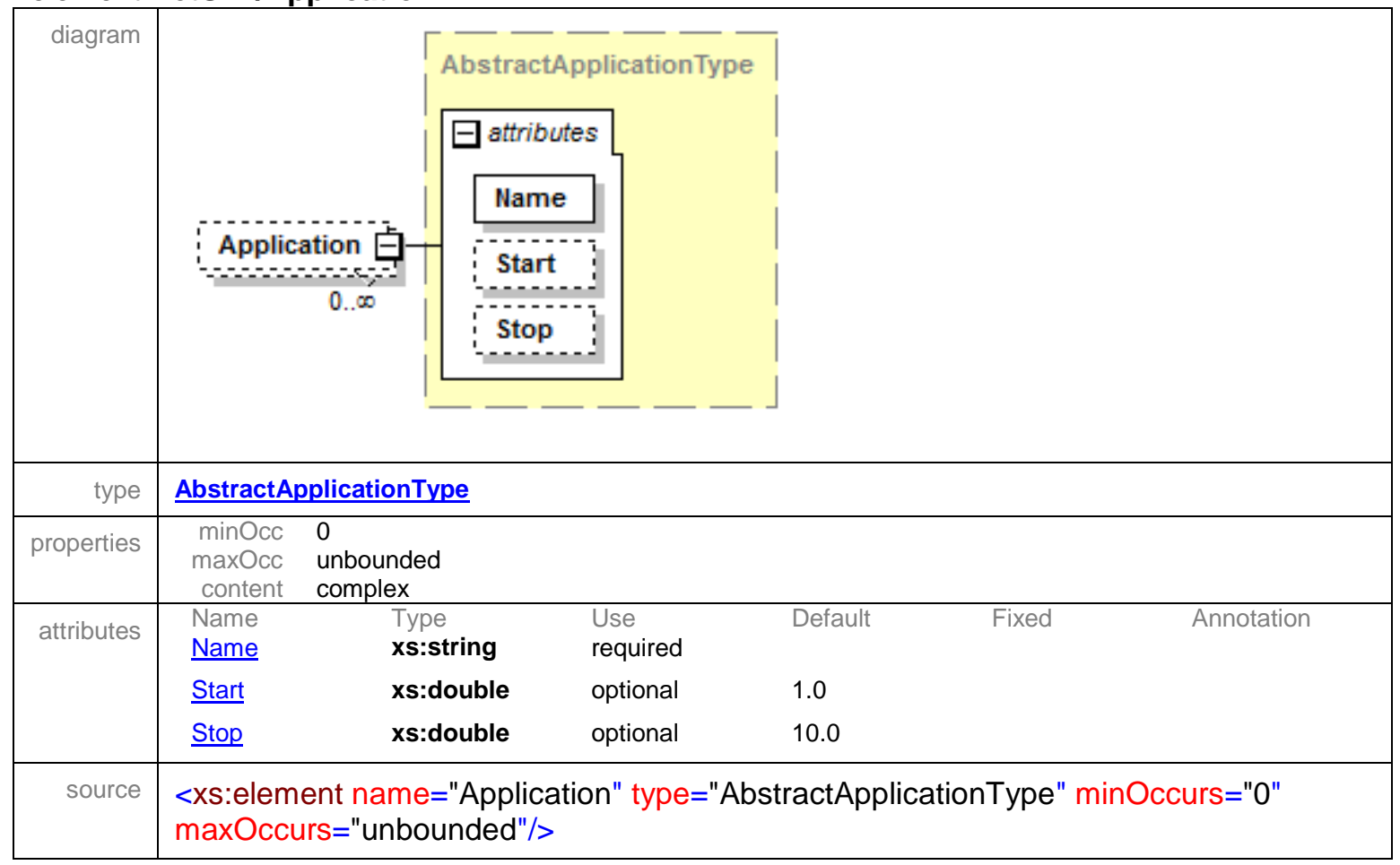

element NetSim/ApplicationSet

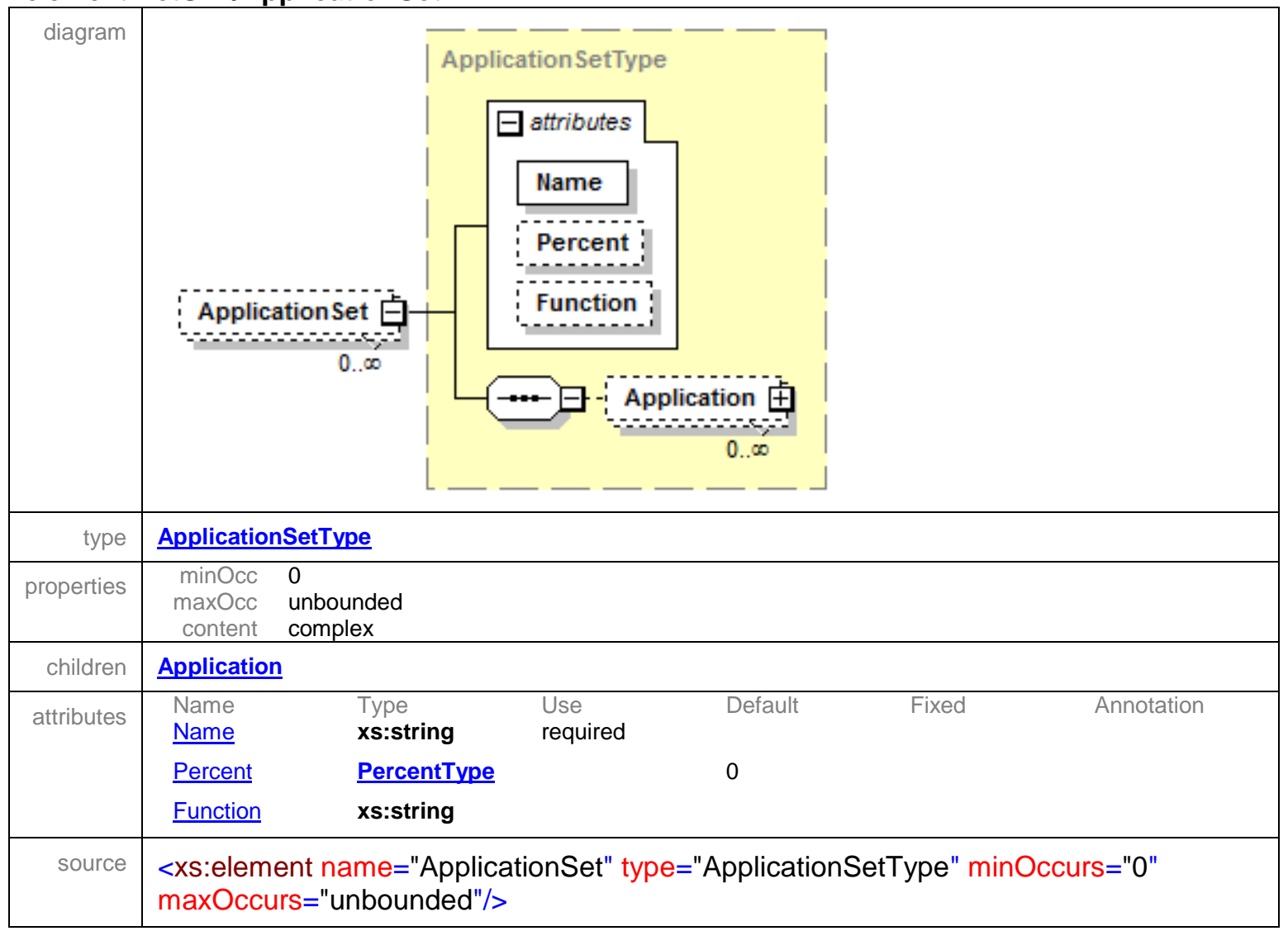


LLNL-TR-630332

complexType AddressRangeType

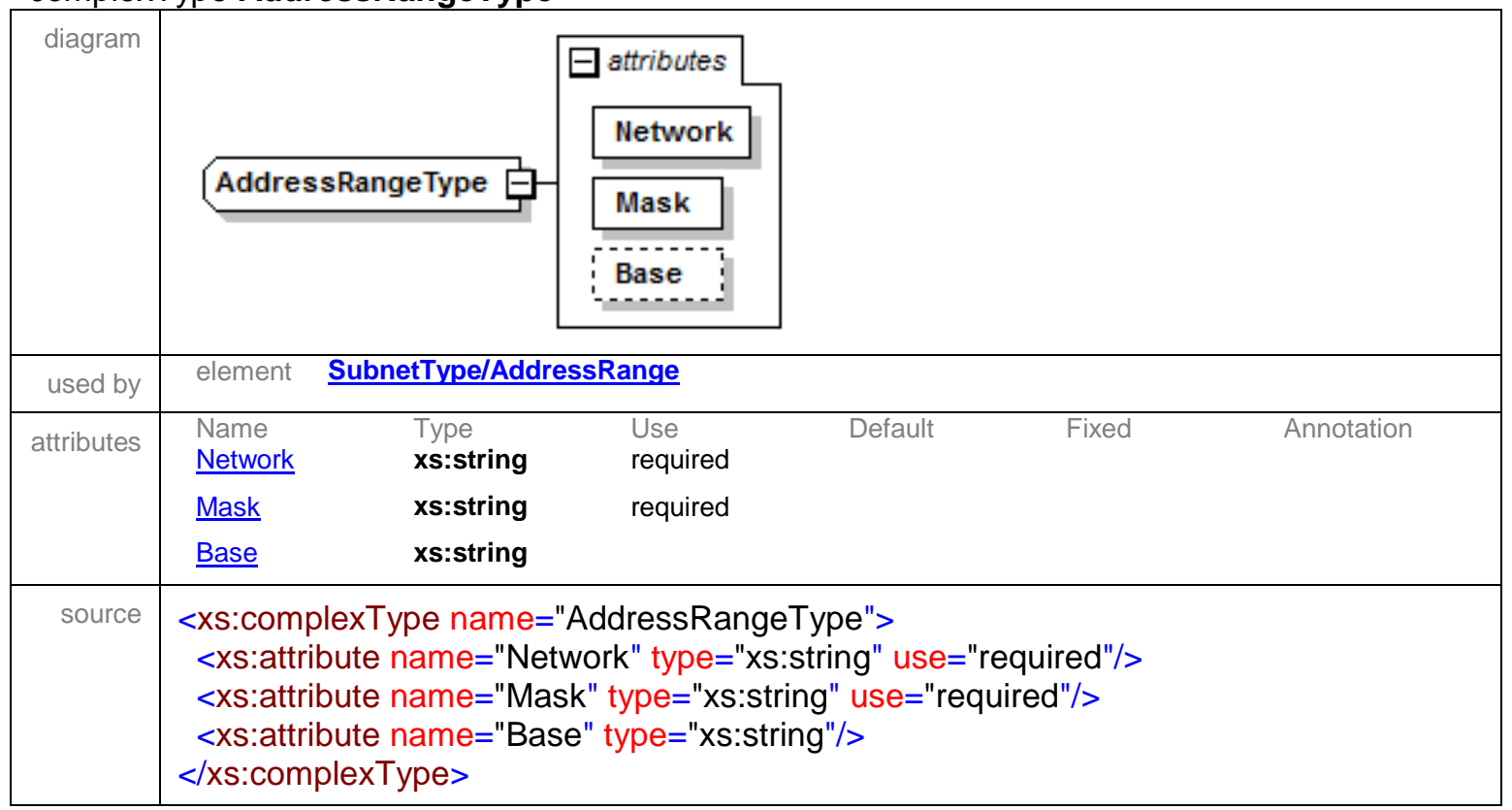

attribute AddressRangeType/@Network

\begin{tabular}{|r|l|}
\hline type & xs:string \\
\hline properties & use required \\
\hline source & $<x s:$ attribute name="Network" type="xs:string" use="required"/> \\
\hline
\end{tabular}

attribute AddressRangeType/@Mask

\begin{tabular}{|r|l|}
\hline type & xs:string \\
\hline properties & use required \\
\hline source & $<x$ s:attribute name="Mask" type="xs:string" use="required"/> \\
\hline
\end{tabular}

attribute AddressRangeType/@Base

\begin{tabular}{|r|l|}
\hline type & xs:string \\
\hline source & $<x s: a t t r i b u t e ~ n a m e=" B a s e " ~ t y p e=" x s:$ string"/> \\
\hline
\end{tabular}

complexType ApplicationRefType

\begin{tabular}{|l|l|l|l|}
\hline diagram & & \\
& ApplicationRefType & (attributes \\
\hline & & Name \\
\hline
\end{tabular}


LLNL-TR-630332

\begin{tabular}{|c|c|c|c|}
\hline used by & element $\quad$ ApplicationSetT & plication & \\
\hline attributes & $\begin{array}{l}\text { Type } \\
\text { xs:string }\end{array}$ & $\begin{array}{l}\text { Use } \\
\text { required }\end{array}$ & Annotation \\
\hline source & \multicolumn{3}{|c|}{$\begin{array}{l}\text { <xs:complexType name="ApplicationRefType"> } \\
\text { <xs:attribute name="Name" type="xs:string" use="required"/> } \\
\text { </xs:complexType> }\end{array}$} \\
\hline
\end{tabular}

attribute ApplicationRefType/@Name

\begin{tabular}{|c|c|}
\hline type & xs:string \\
\hline properties & use required \\
\hline source & <xs:attribute name="Name" type="xs:string" use="required"/> \\
\hline
\end{tabular}

complexType ApplicationSetType

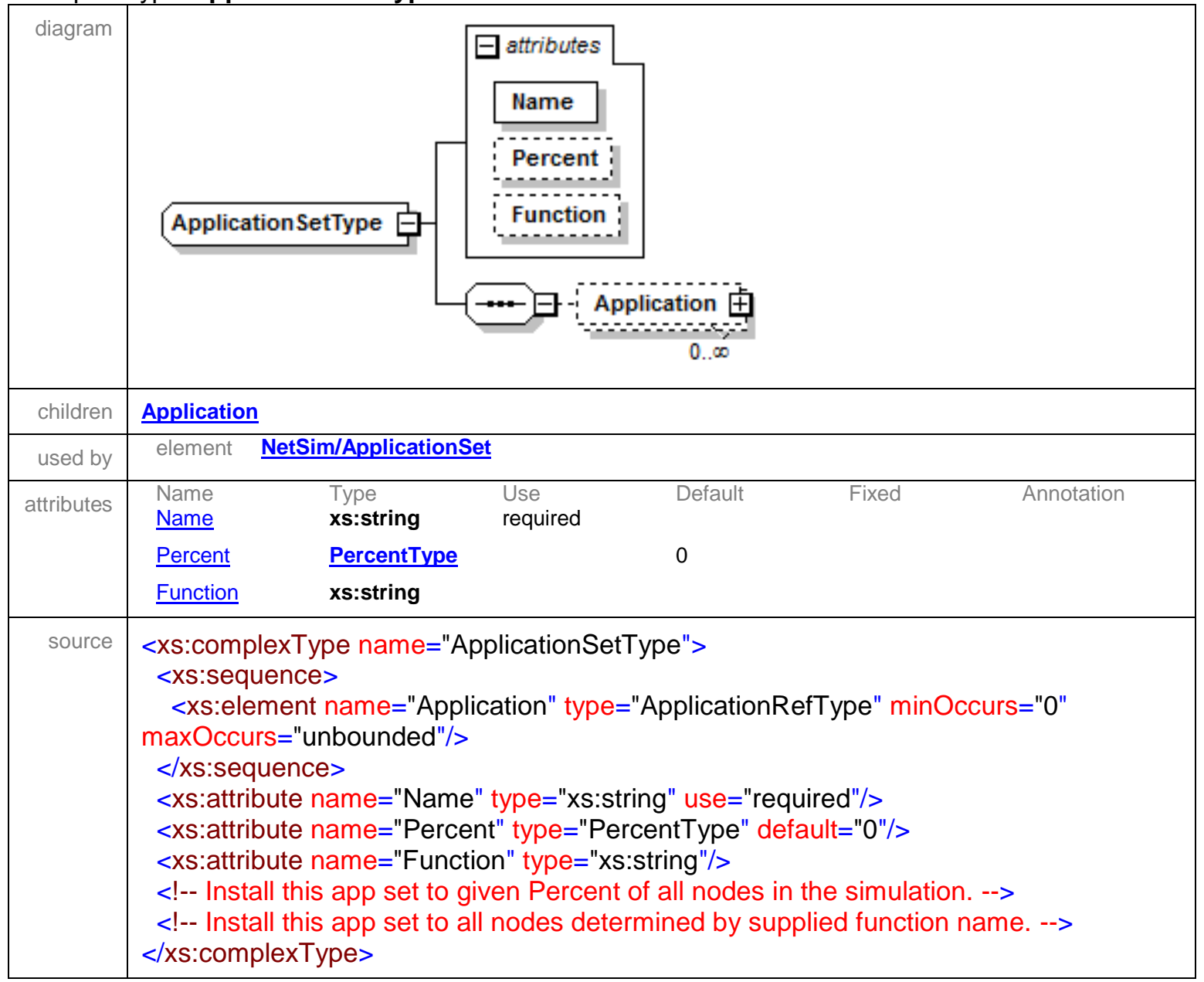

attribute ApplicationSetType/@Name

type $\mathbf{x s}$ :string 
LLNL-TR-630332

\begin{tabular}{|r|c|}
\hline properties & use required \\
\hline source & $<x$ s:attribute name="Name" type="xs:string" use="required"/> \\
\hline
\end{tabular}

attribute ApplicationSetType/@Percent

\begin{tabular}{|r|l|}
\hline type & PercentType \\
\hline properties & default 0 \\
\hline facets & $\begin{array}{l}\text { Kind } \\
\text { minInclusive } \\
\text { maxInclusive } \quad 100\end{array}$ \\
\hline source & $<x$ :attribute name="Percent" type="PercentType" default="0"/> \\
\hline
\end{tabular}

attribute ApplicationSetType/@Function

\begin{tabular}{|r|l|}
\hline type & xs:string \\
\hline source & $<x s: a t t r i b u t e ~ n a m e=" F u n c t i o n " ~ t y p e=" x s: s t r i n g " />$ \\
\hline
\end{tabular}

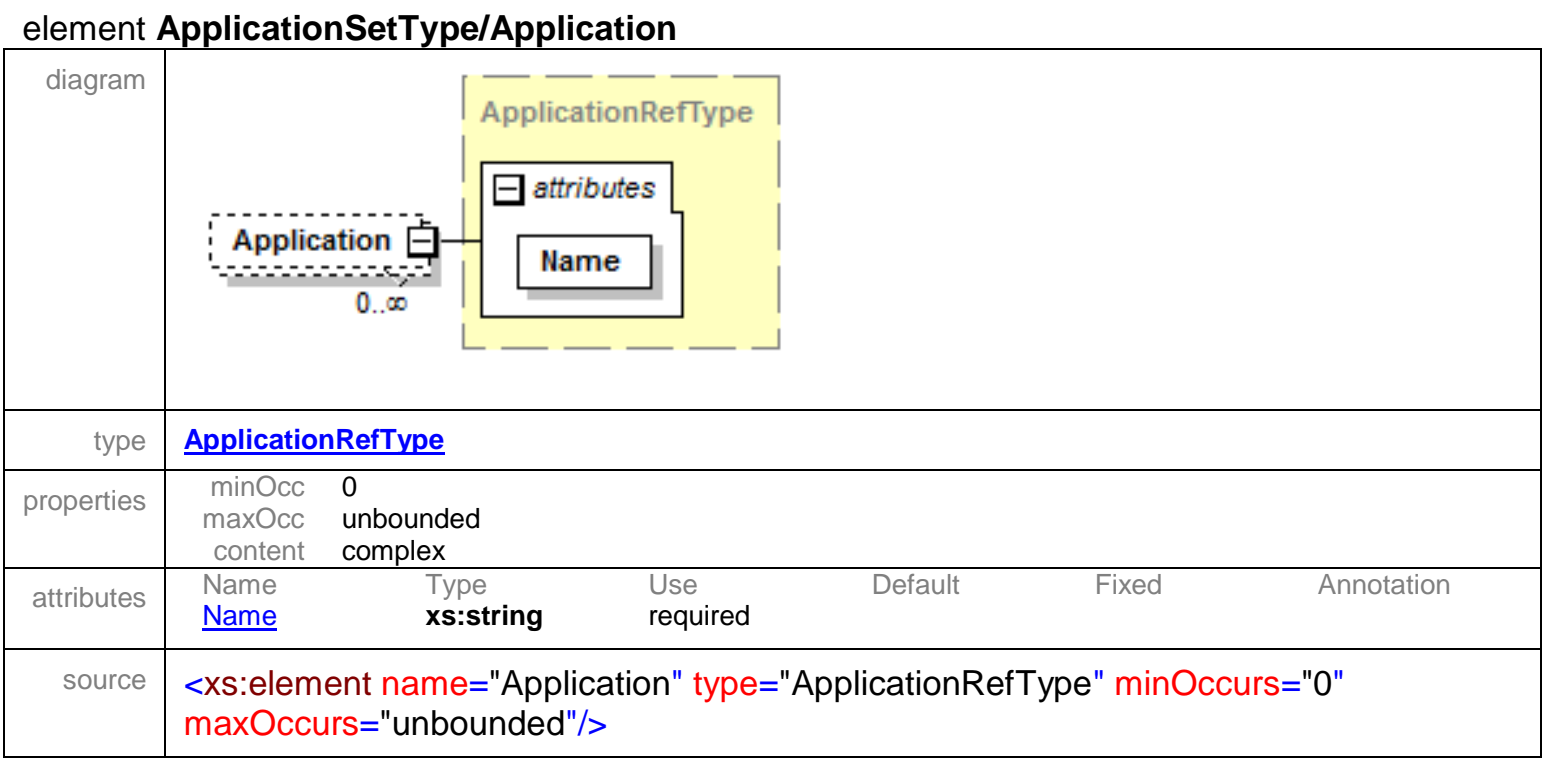

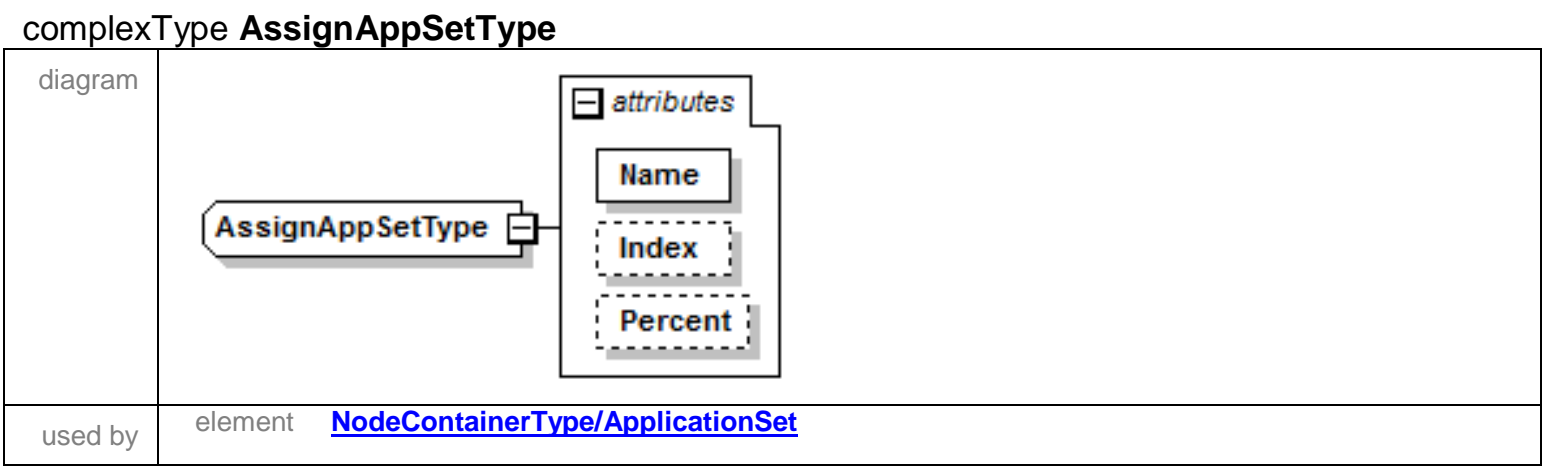


LLNL-TR-630332

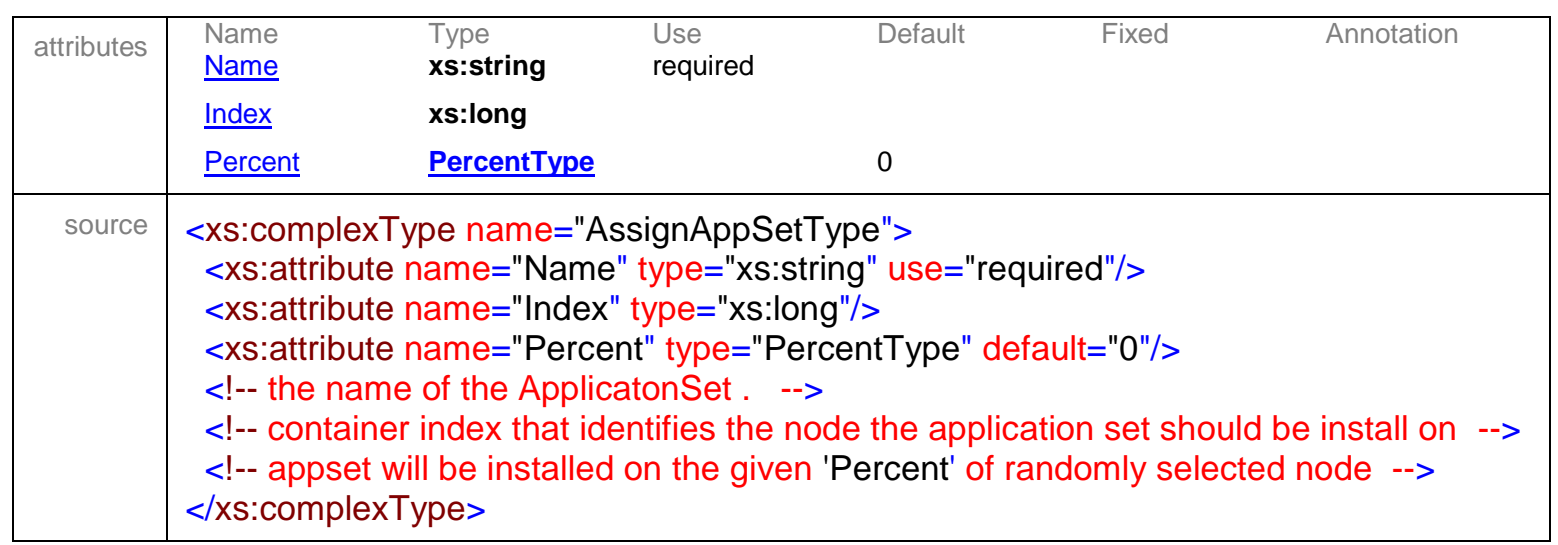

attribute AssignAppSetType/@Name

\begin{tabular}{|c|c|}
\hline type & xs:string \\
\hline properties & use required \\
\hline source & <xs:attribute name="Name" type="xs:string" use="required"/> \\
\hline
\end{tabular}

attribute AssignAppSetType/@Index

\begin{tabular}{|r|l|}
\hline type & xs:long \\
\hline source & $<x$ :attribute name="Index" type="xs:long"/> \\
\hline
\end{tabular}

attribute AssignAppSetType/@Percent

\begin{tabular}{|r|l|}
\hline type & PercentType \\
\hline properties & default 0 \\
\hline facets & $\begin{array}{l}\text { Kind } \\
\text { minlnclusive } 0 \\
\text { maxInclusive } 100\end{array}$ \\
\hline source & $<x$ :attribute name="Percent" type="PercentType" default="0"/> \\
\hline
\end{tabular}


LLNL-TR-630332

complexType NodeContainerType

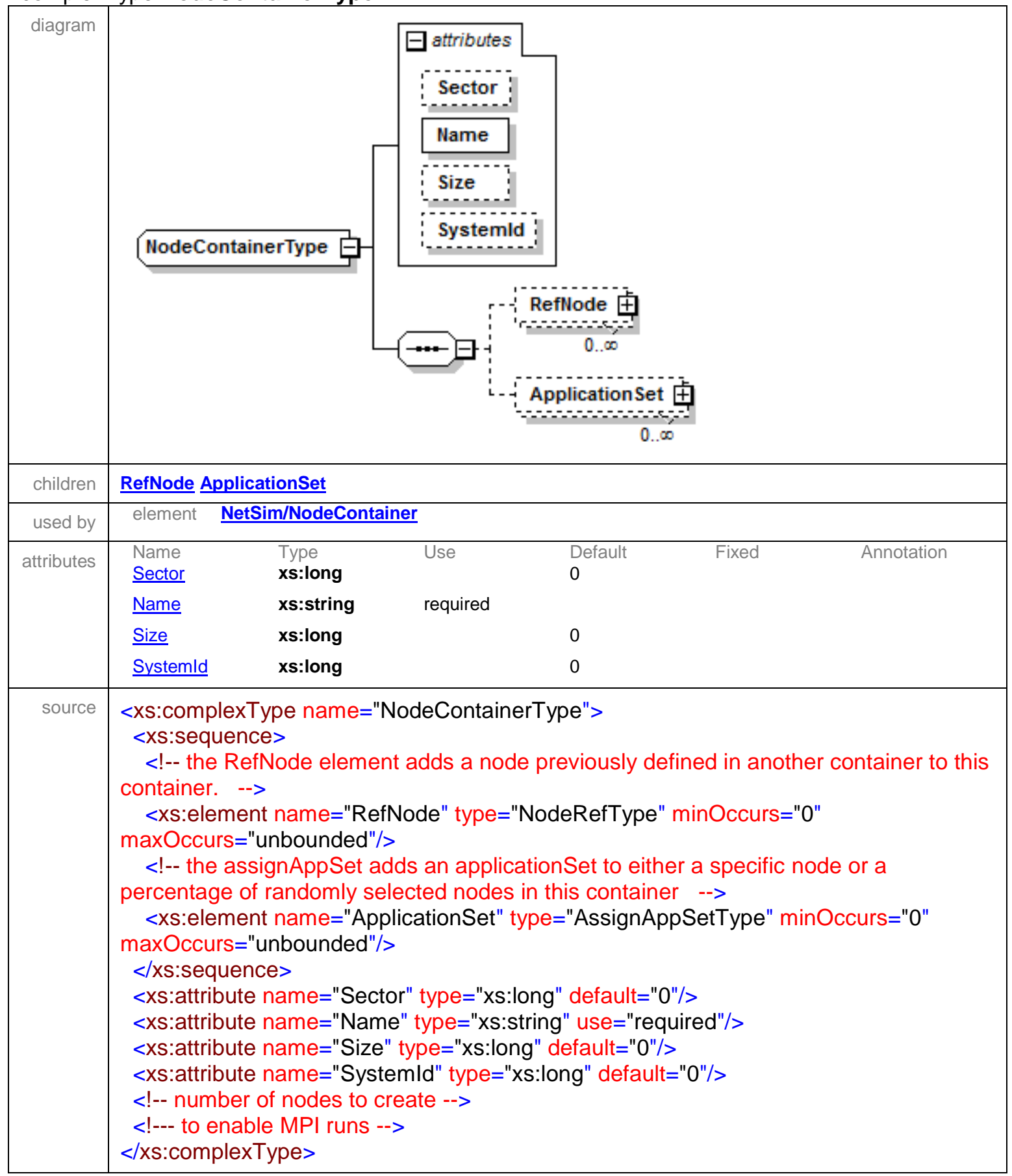

attribute NodeContainerType/@Sector

\begin{tabular}{|r|l|}
\hline type & xs:long \\
\hline properties & default 0 \\
\hline source & $<x$ :attribute name="Sector" type="xs:long" default="0"/> \\
\hline
\end{tabular}


LLNL-TR-630332

attribute NodeContainerType/@Name

\begin{tabular}{|r|l|}
\hline type & xs:string \\
\hline properties & use required \\
\hline source & $<x s:$ attribute name="Name" type="xs:string" use="required"/> \\
\hline
\end{tabular}

attribute NodeContainerType/@Size

\begin{tabular}{|r|l|}
\hline type & xs:long \\
\hline properties & default 0 \\
\hline source & $<x$ :attribute name="Size" type="xs:long" default="0"/> \\
\hline
\end{tabular}

attribute NodeContainerType/@Systemld

\begin{tabular}{|c|c|}
\hline type & xs:long \\
\hline properties & default 0 \\
\hline source & $<x$ :attribute name="Systemld" type="xs:long" default="0"/> \\
\hline
\end{tabular}

element NodeContainerType/RefNode

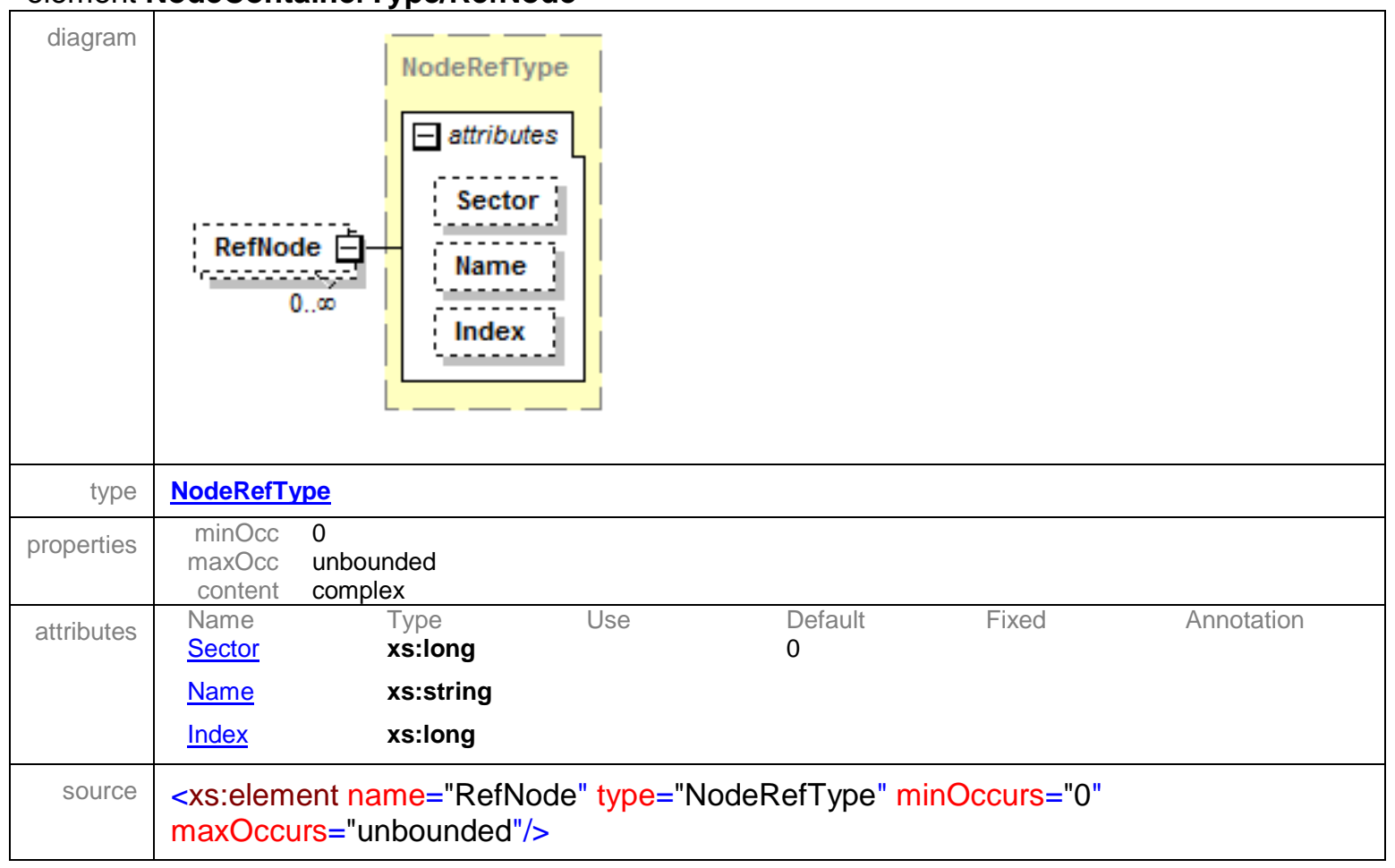


element NodeContainerType/ApplicationSet

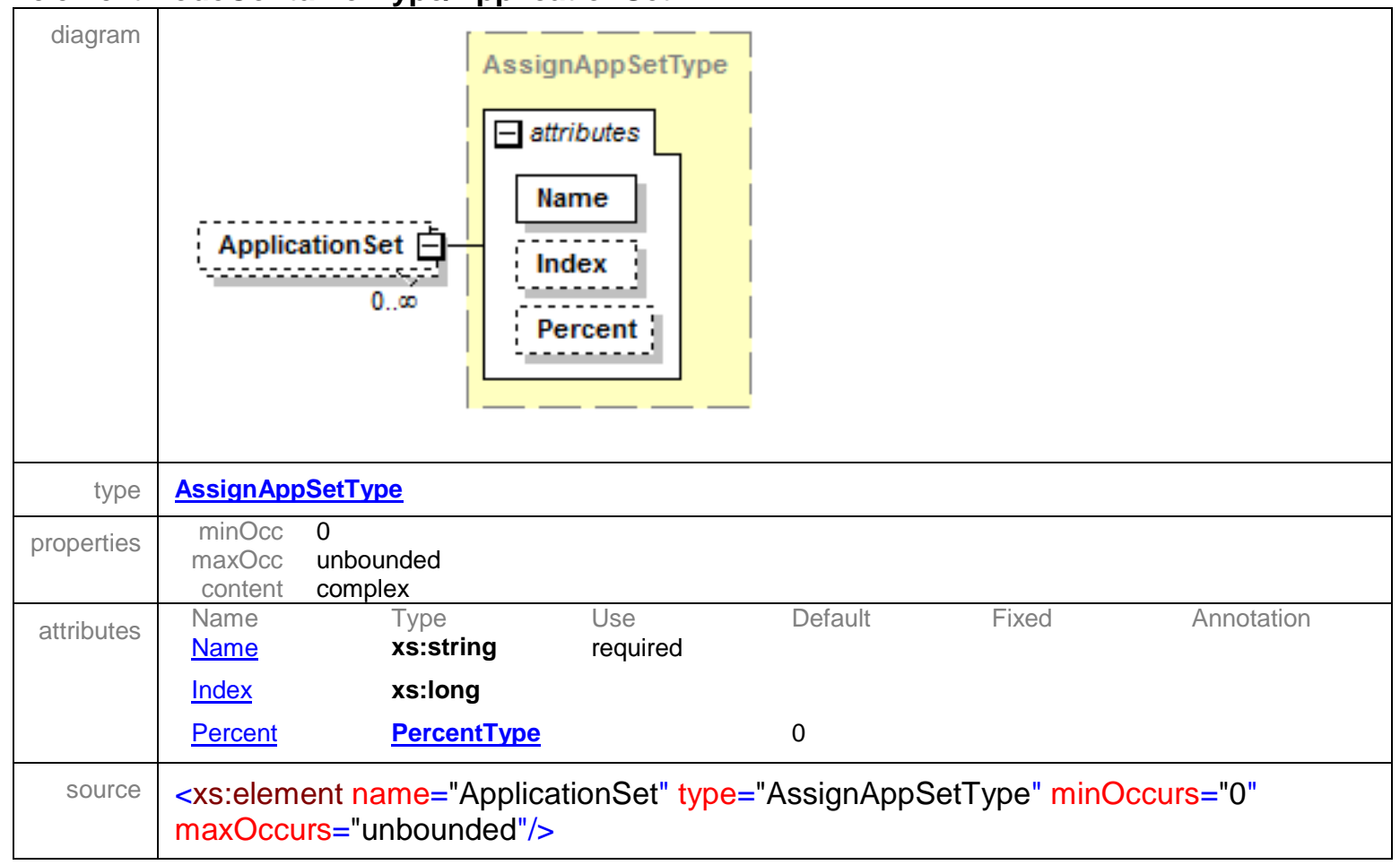

complexType NodeRefType

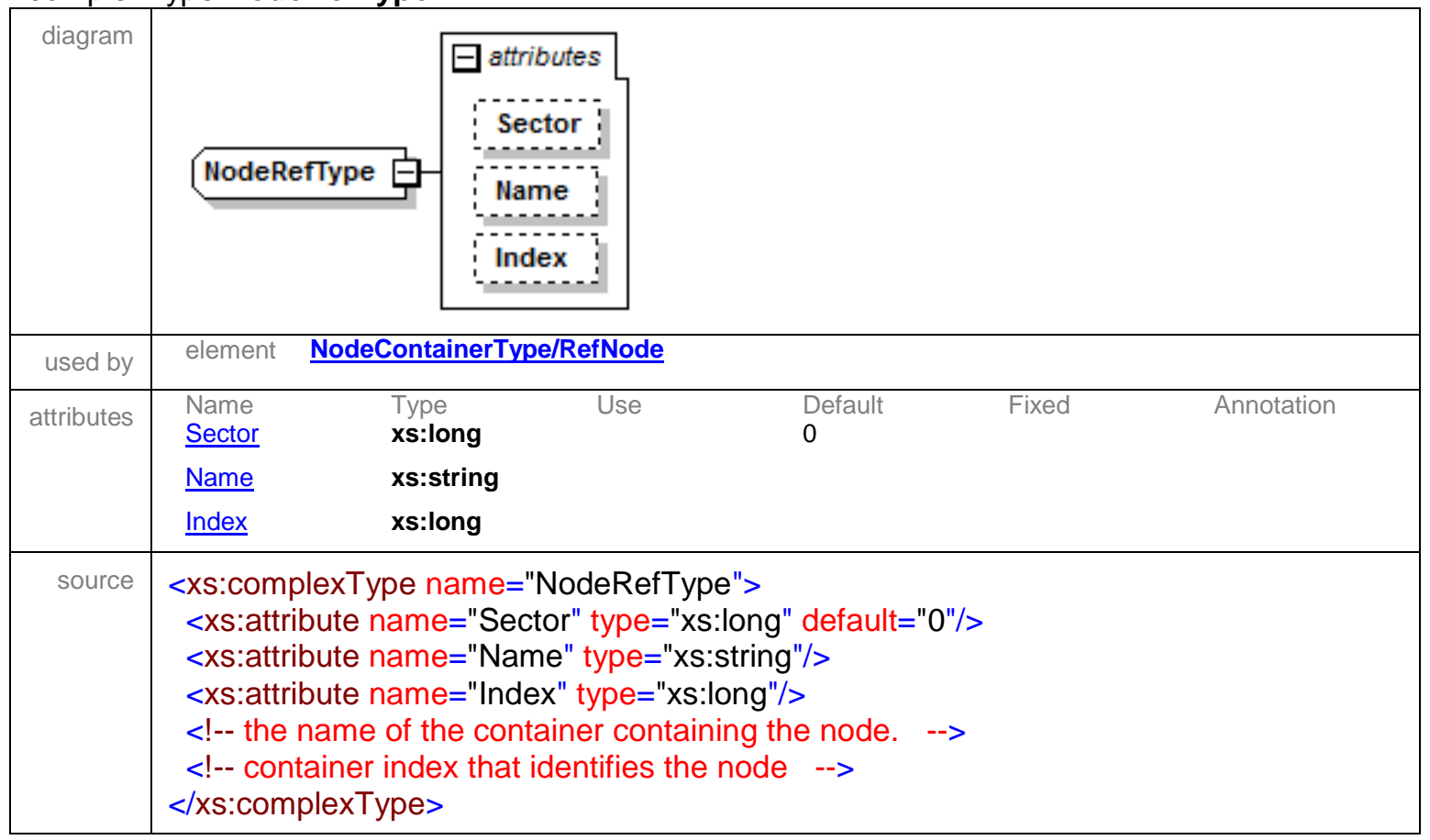

attribute NodeRefType/@Sector 
LLNL-TR-630332

\begin{tabular}{|r|c|}
\hline properties & default 0 \\
\hline source & $<x$ s:attribute name="Sector" type="xs:long" default="0"/> \\
\hline
\end{tabular}

attribute NodeRefType/@Name

\begin{tabular}{|r|l|}
\hline type & xs:string \\
\hline source & $<x s: a t t r i b u t e ~ n a m e=" N a m e "$ type="xs:string"/> \\
\hline
\end{tabular}

attribute NodeRefType/@Index

\begin{tabular}{|r|l|}
\hline type & xs:long \\
\hline source & $<x s: a t t r i b u t e ~ n a m e=" I n d e x "$ type="xs:long"/> \\
\hline
\end{tabular}

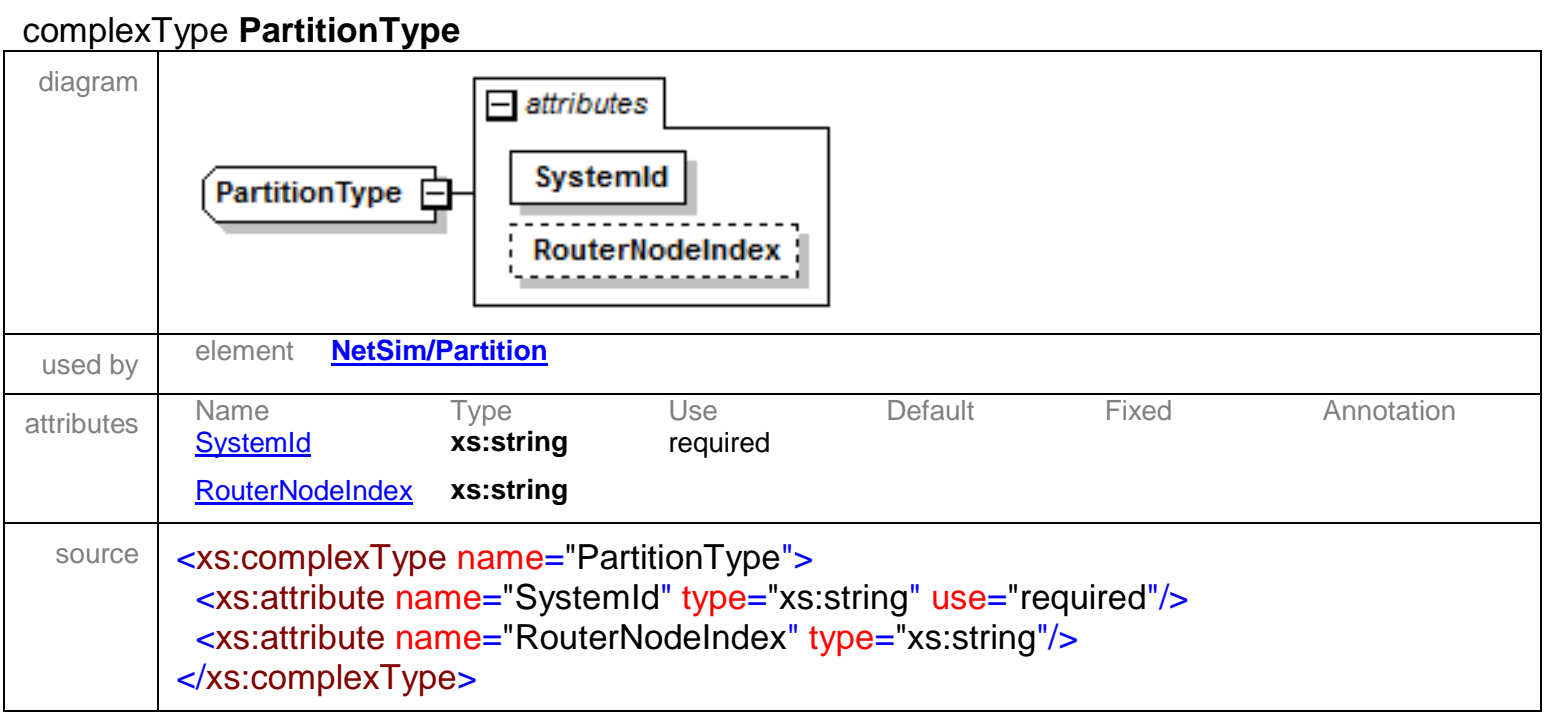

attribute PartitionType/@Systemld

\begin{tabular}{|r|l|}
\hline type & xs:string \\
\hline properties & use required \\
\hline source & $<x s:$ attribute name="Systemld" type="xs:string" use="required"/> \\
\hline
\end{tabular}

attribute PartitionType/@RouterNodelndex

\begin{tabular}{|r|l|}
\hline type & xs:string \\
\hline source & $<x$ :attribute name="RouterNodelndex" type="xs:string"/> \\
\hline
\end{tabular}


LLNL-TR-630332

complexType RefNodelnterfaceDefinitionType

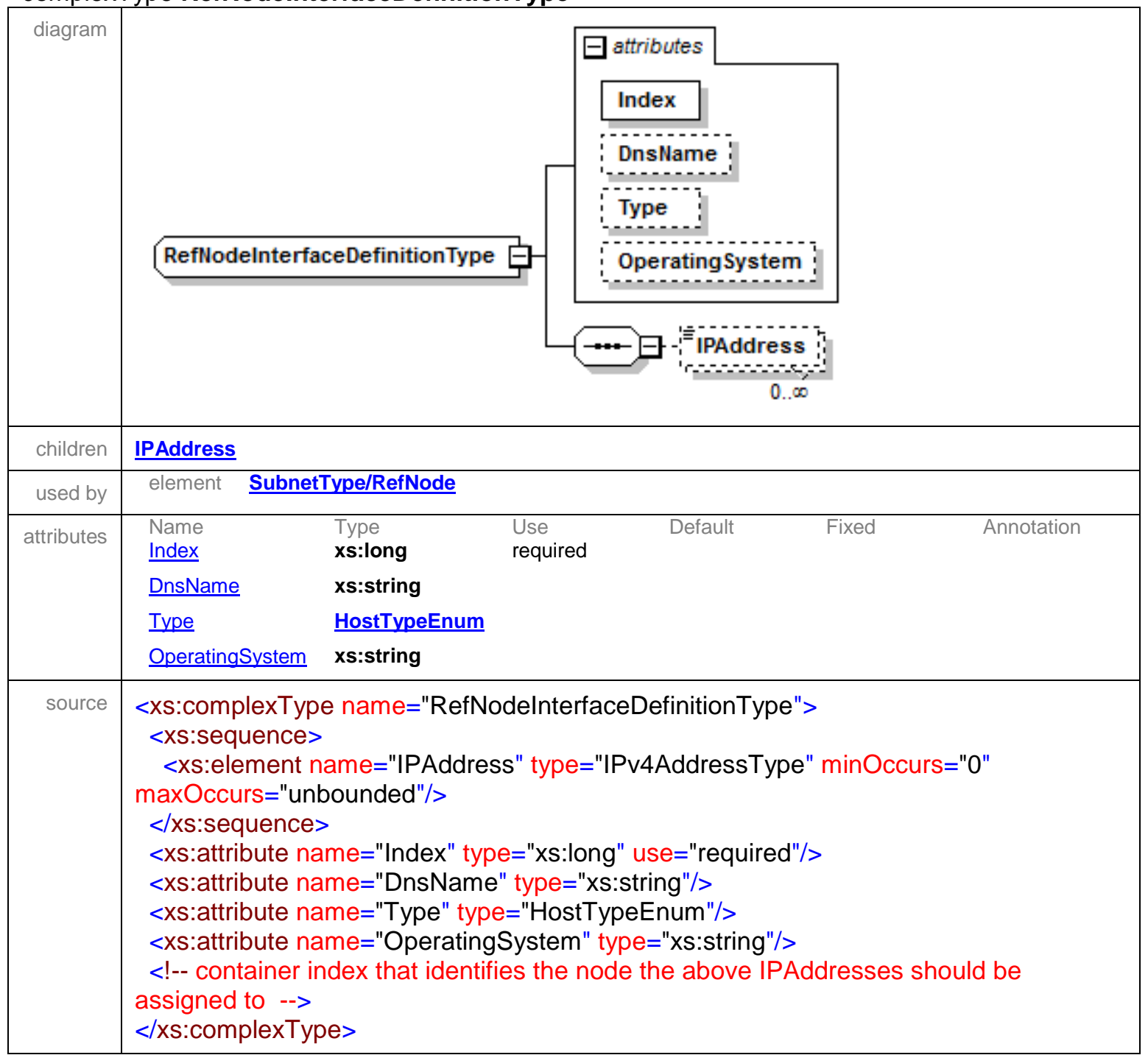

attribute RefNodelnterfaceDefinitionType/@Index

\begin{tabular}{|r|l|}
\hline type & xs:long \\
\hline properties & use required \\
\hline source & $<x s:$ attribute name="Index" type="xs:long" use="required"/> \\
\hline
\end{tabular}

attribute RefNodelnterfaceDefinitionType/@DnsName

\begin{tabular}{|r|l|}
\hline type & xs:string \\
\hline source & $<x$ :attribute name="DnsName" type="xs:string"/> \\
\hline
\end{tabular}

attribute RefNodelnterfaceDefinitionType/@Type 
LLNL-TR-630332

\begin{tabular}{|c|ll|}
\hline \multirow{2}{*}{ facets } & $\begin{array}{l}\text { Kind } \\
\text { enumeration }\end{array}$ & Value \\
& $\begin{array}{l}\text { SIMPLE } \\
\text { enumeration }\end{array}$ & SWITCH \\
& enumeration & ROUTER \\
\hline source & $<$ xs:attribute name="Type" type="HostTypeEnum"/> \\
\hline
\end{tabular}

attribute RefNodelnterfaceDefinitionType/@OperatingSystem

\begin{tabular}{|r|l|}
\hline type & xs:string \\
\hline source & $<x s: a t t r i b u t e ~ n a m e=" O p e r a t i n g S y s t e m "$ type="xs:string"/> \\
\hline
\end{tabular}

element RefNodelnterfaceDefinitionType/IPAddress

\begin{tabular}{|c|c|c|}
\hline diagram & 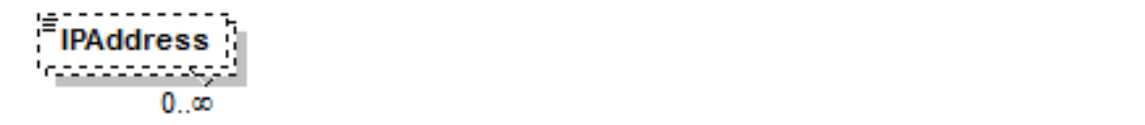 & \\
\hline type & IPv4AddressType & \\
\hline properties & $\begin{aligned} & \operatorname{minOcc} 0 \\
& \text { maxOcc } \text { unbounded } \\
& \text { content } \text { simple } \\
&\end{aligned}$ & \\
\hline facets & $\begin{array}{ll}\text { Kind } & \text { Value } \\
\text { pattern } & ((25[0-5]|2[0-4][0-9]| 1[0-9][0-9]|[1-9][0-9]|[0-9]) \backslash .)\{3\}(25[0-5]|2[0-4][0-9]| 1[0-9][0- \\
& 9]|[1-9][0-9]|[0-9])\end{array}$ & Annotation \\
\hline source & $\begin{array}{l}<x s: \text { element name="IPAddress" type="IPv4AddressType" minOccurs="0" } \\
\text { maxOccurs="unbounded"/> }\end{array}$ & \\
\hline
\end{tabular}

complexType SimType

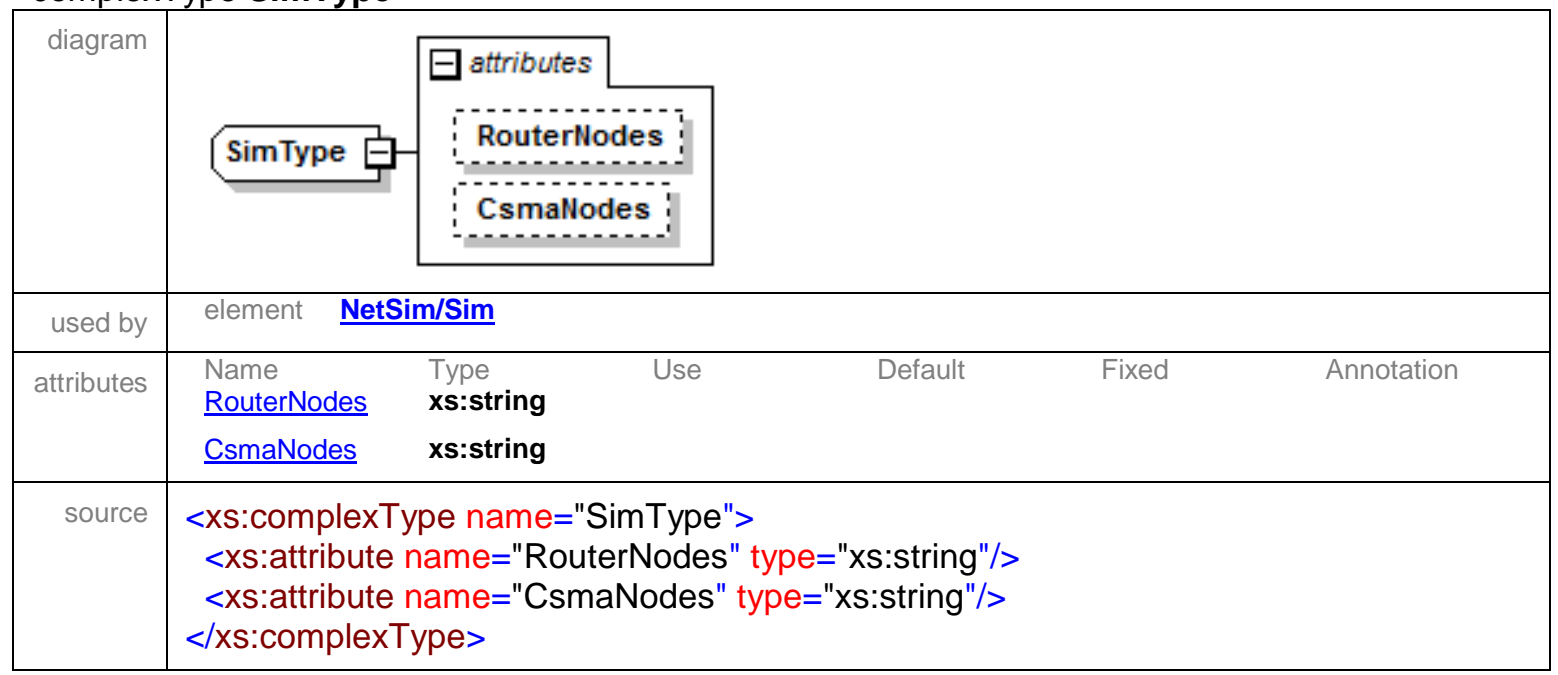

attribute SimType/@RouterNodes

\begin{tabular}{|c|c|}
\hline type & xs:string \\
\hline source & <xs:attribute name="RouterNodes" type="xs:string"/> \\
\hline
\end{tabular}


LLNL-TR-630332

attribute SimType/@CsmaNodes

\begin{tabular}{|r|l|}
\hline type & xs:string \\
\hline source & $<x s:$ attribute name="CsmaNodes" type="xs:string"/> \\
\hline
\end{tabular}

complexType SubnetType

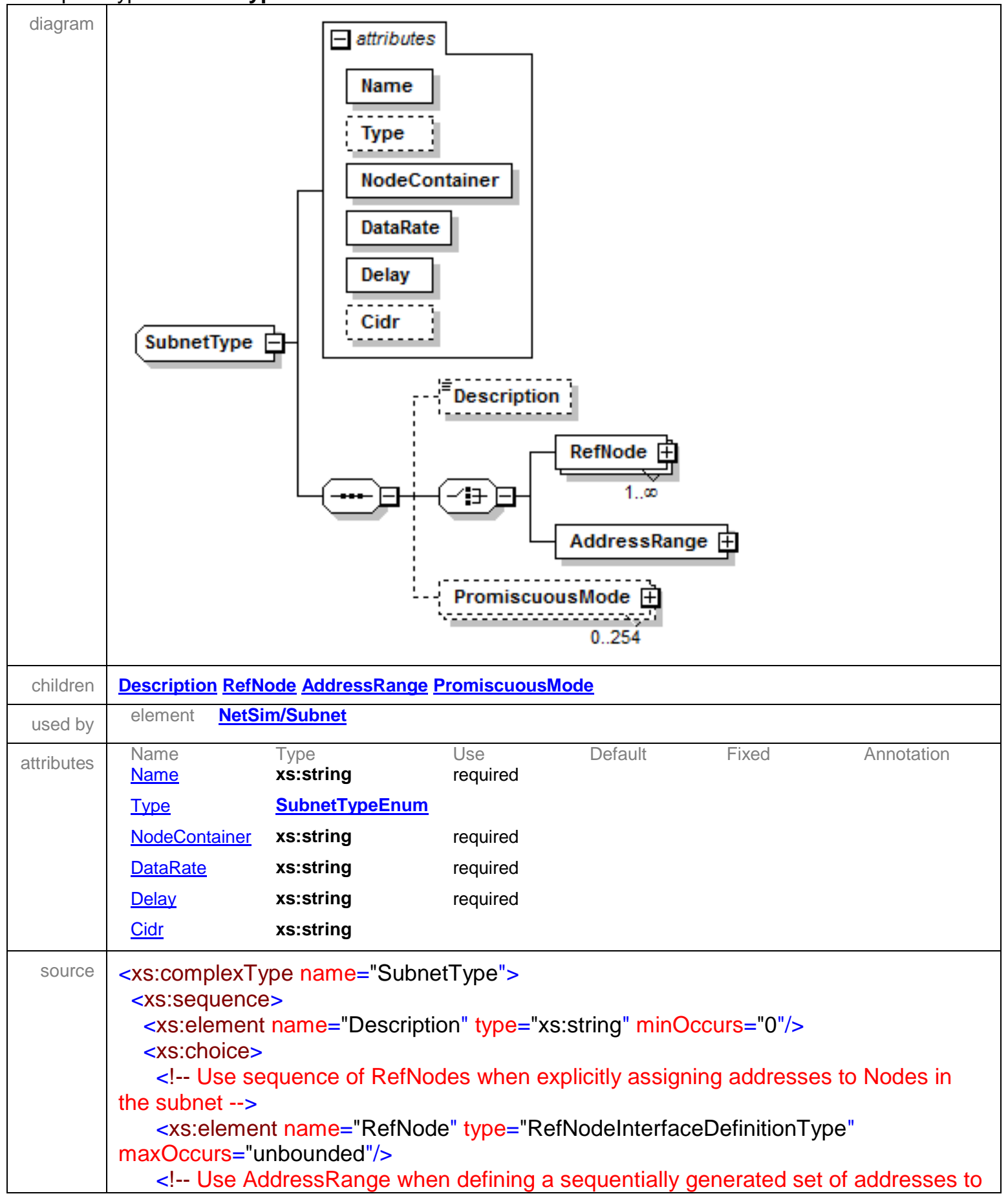




\begin{tabular}{|l|}
\hline assign to nodes in the subnet --> \\
<xs:element name="AddressRange" type="AddressRangeType"/> \\
</xs:choice> \\
<!-- This is an optional list of indexes of interfaces within the defined network range \\
that should be put into promiscuous mode --> \\
<xs:element name="PromiscuousMode" type="PromiscuousModeType" \\
minOccurs="0" maxOccurs="254"/> \\
<xs:sequence> \\
<xs:attribute name="Name" type="xs:string" use="required"/> \\
<xs:attribute name="Type" type="SubnetTypeEnum"/> \\
<xs:attribute name="NodeContainer" type="xs:string" use="required"/> \\
<xs:attribute name="DataRate" type="xs:string" use="required"/> \\
<xs:attribute name="Delay" type="xs:string" use="required"/> \\
<xs:attribute name="Cidr" type="xs:string"/> \\
<xs:complexType>
\end{tabular}

attribute SubnetType/@Name

\begin{tabular}{|c|c|}
\hline type & xs:string \\
\hline properties & use required \\
\hline source & <xs:attribute name="Name" type="xs:string" use="required"/> \\
\hline
\end{tabular}

attribute SubnetType/@Type

\begin{tabular}{|r|l|}
\hline type & \multicolumn{2}{|c|}{ SubnetTypeEnum } \\
\hline \multirow{2}{*}{ facets } & $\begin{array}{l}\text { Kind } \\
\text { enumeration Calue Annotation } \\
\text { enumeration P2P }\end{array}$ \\
\hline source & $<$ xs:attribute name="Type" type="SubnetTypeEnum"/> \\
\hline
\end{tabular}

attribute SubnetType/@NodeContainer

\begin{tabular}{|r|l|}
\hline type & xs:string \\
\hline properties & use required \\
\hline source & $<x s$ :attribute name="NodeContainer" type="xs:string" use="required"/> \\
\hline
\end{tabular}

attribute SubnetType/@DataRate

\begin{tabular}{|r|c|}
\hline type & xs:string \\
\hline properties & use required \\
\hline source & $<x$ :attribute name="DataRate" type="xs:string" use="required"/> \\
\hline
\end{tabular}

attribute SubnetType/@Delay

\begin{tabular}{|r|l|}
\hline type & xs:string \\
\hline properties & use required \\
\hline
\end{tabular}


LLNL-TR-630332

source

<xs:attribute name="Delay" type="xs:string" use="required"/>

attribute SubnetType/@Cidr

\begin{tabular}{|r|l|}
\hline type & xs:string \\
\hline source & $<x s: a t t r i b u t e ~ n a m e=" C i d r "$ type="xs:string"/> \\
\hline
\end{tabular}

element SubnetType/Description

\begin{tabular}{|r|c|}
\hline diagram & \\
\hline type & xs:string \\
\hline properties & $\begin{array}{r}\text { minOcc } \\
\text { maxOcc } \\
\text { content } \quad \text { simple }\end{array}$ \\
\hline source & $<x$ :element name="Description" type="xs:string" minOccurs="0"/> \\
\hline
\end{tabular}

element SubnetType/RefNode

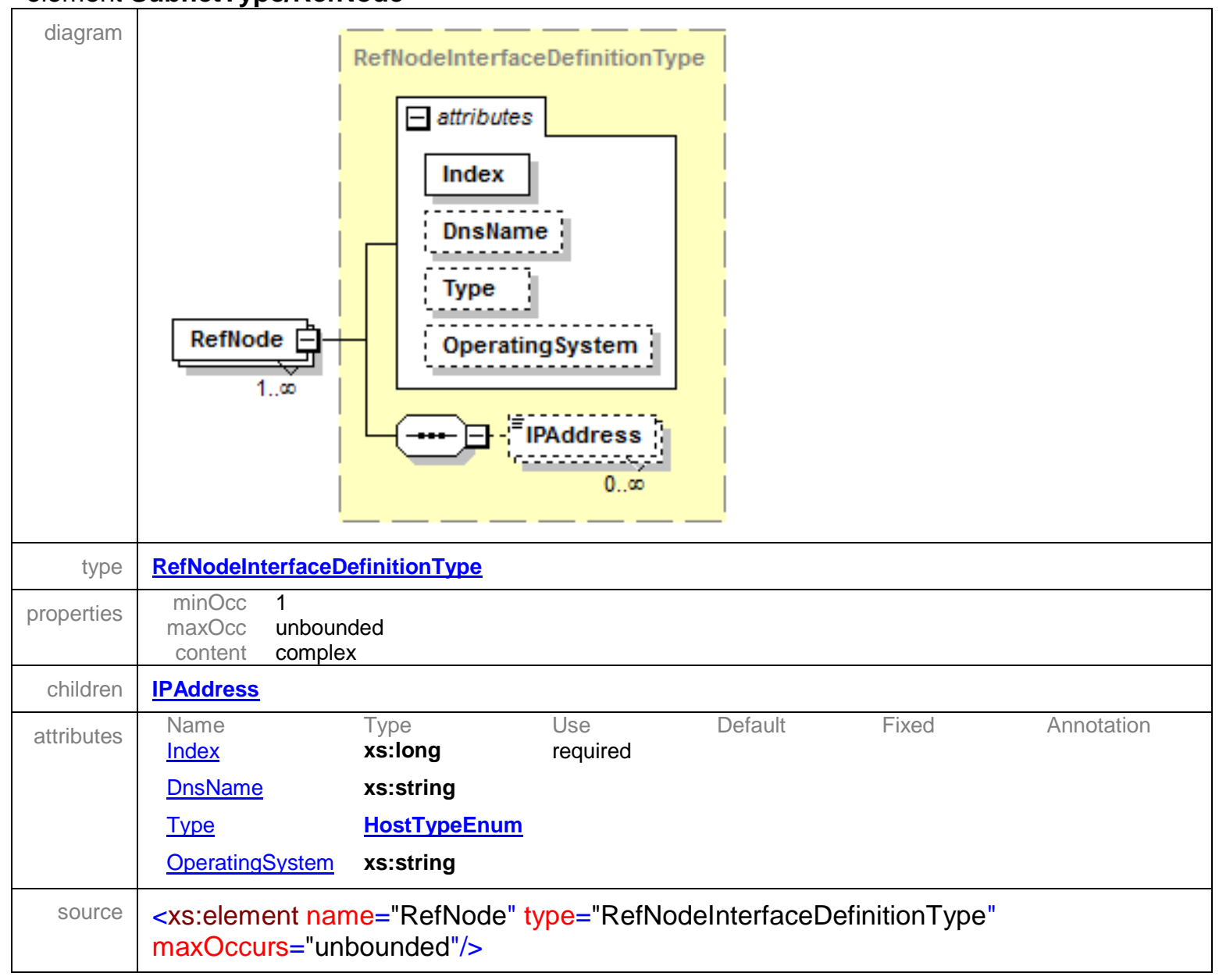


LLNL-TR-630332

element SubnetType/AddressRange

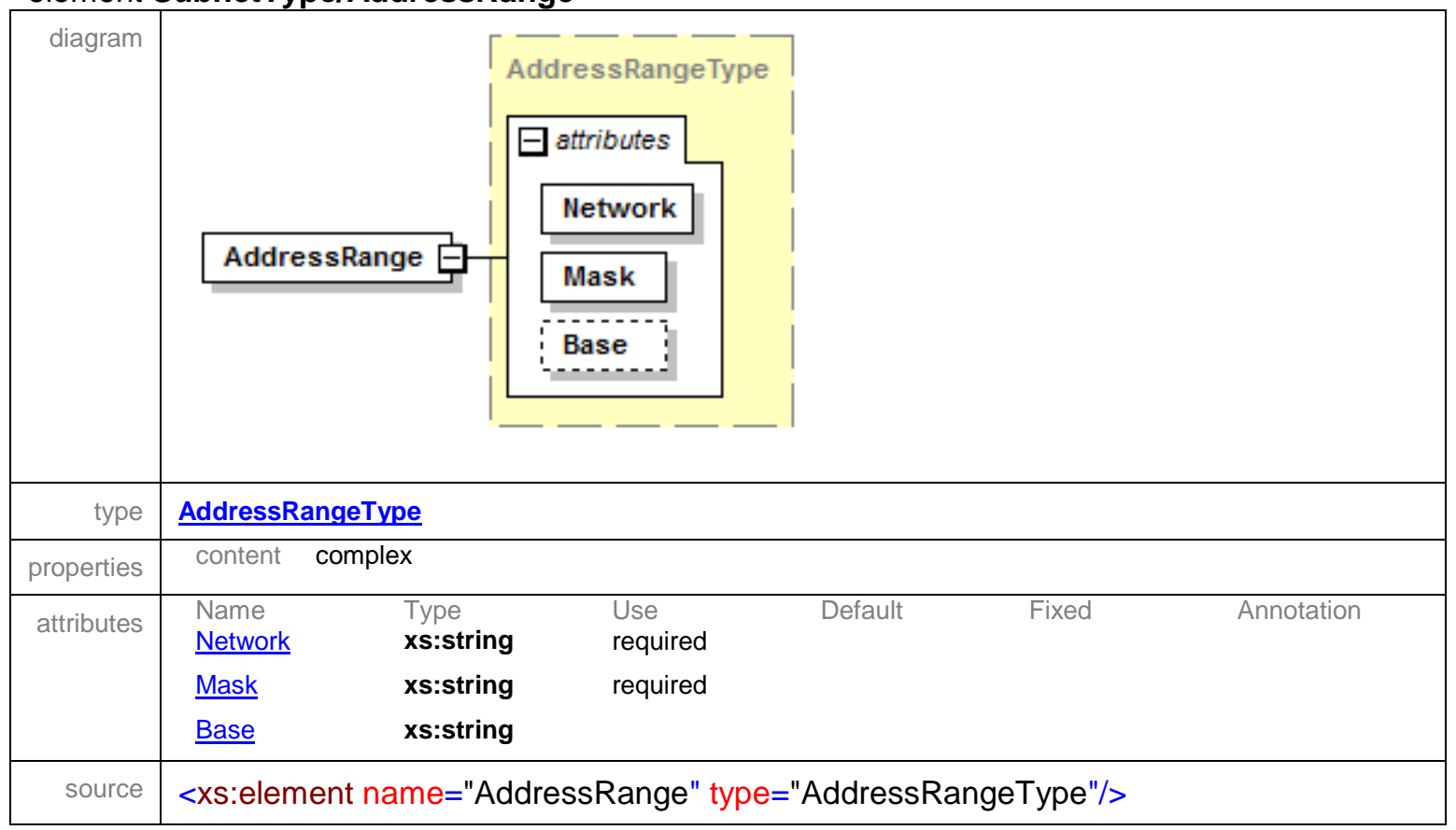

element SubnetType/PromiscuousMode

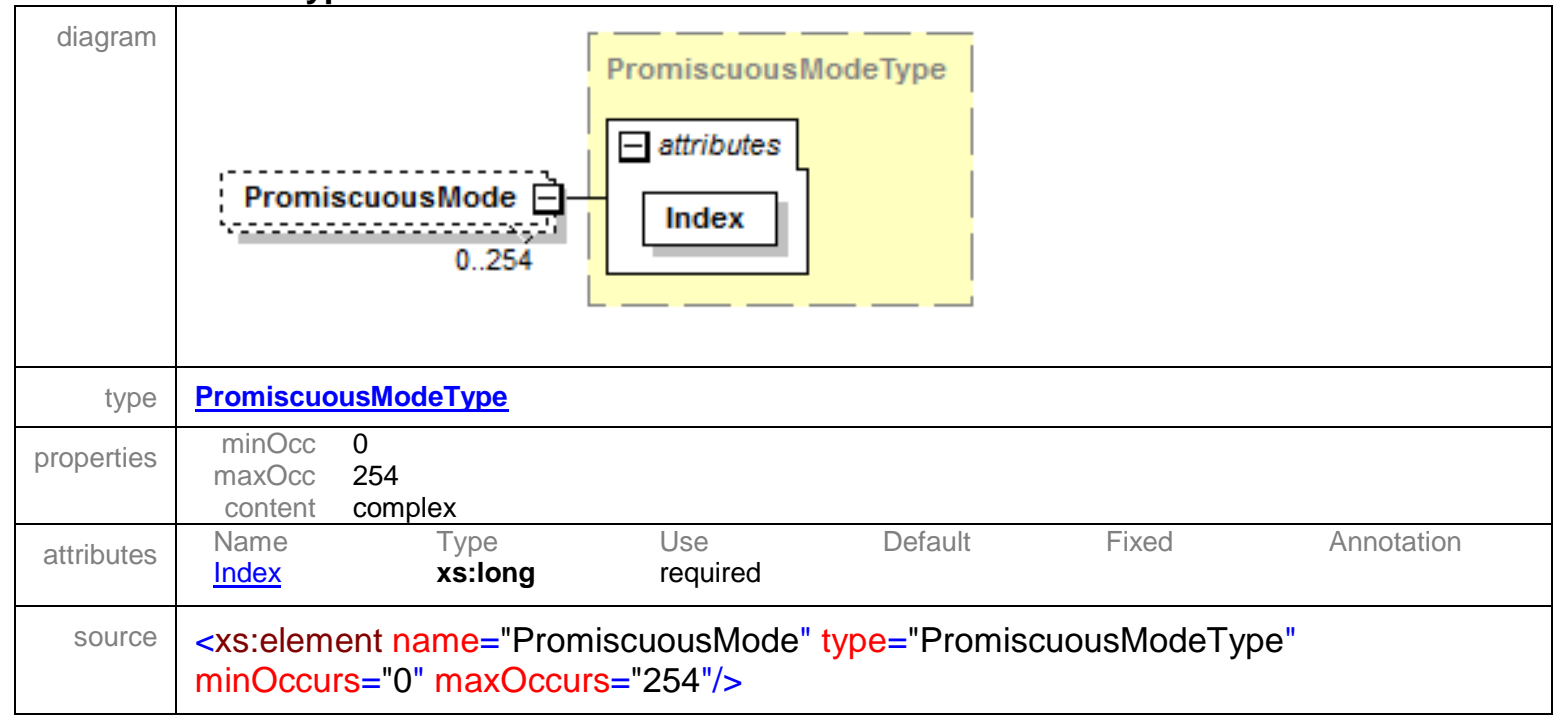


LLNL-TR-630332

complexType AbstractApplicationType

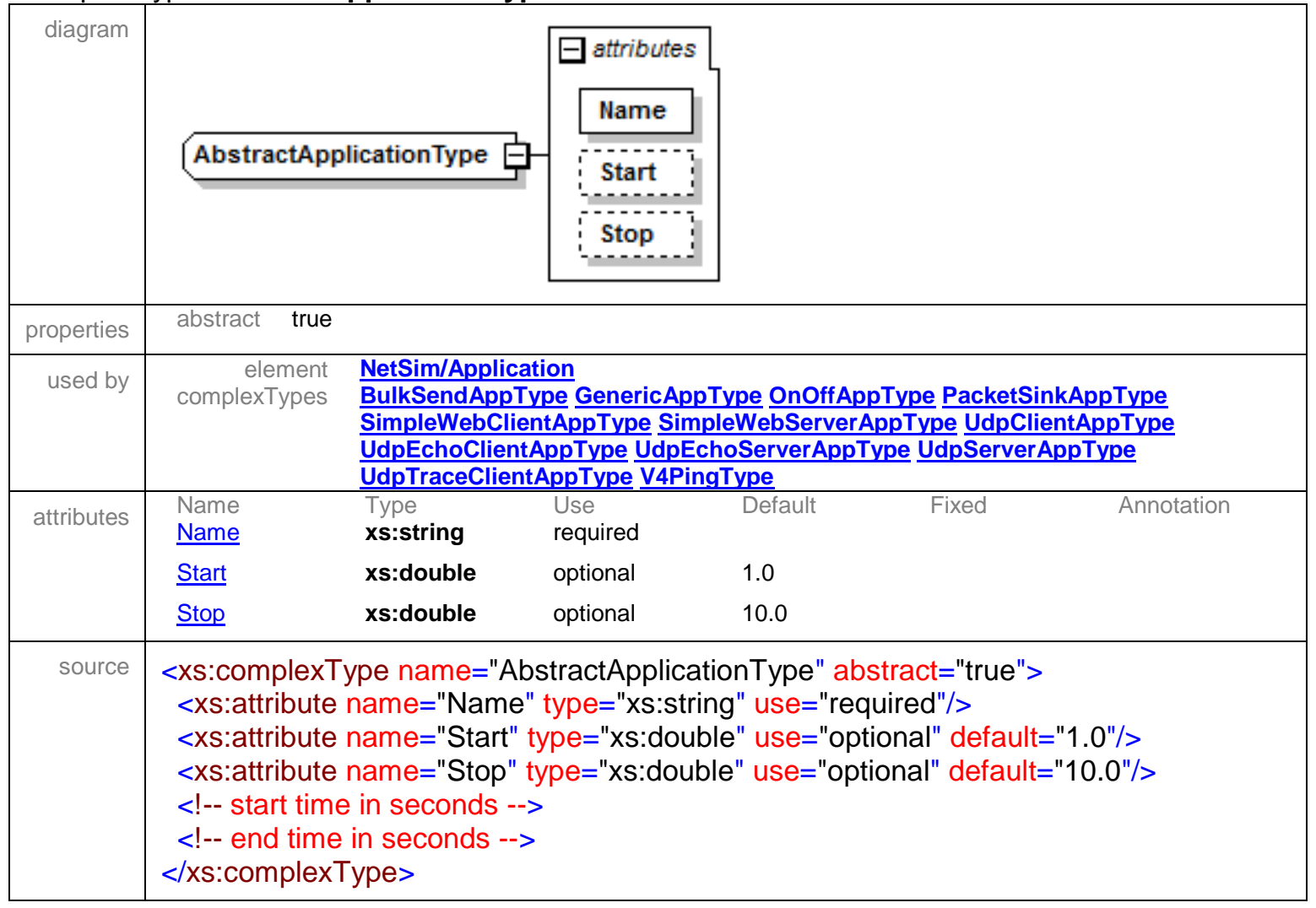

attribute AbstractApplicationType/@Name

\begin{tabular}{|c|c|}
\hline type & xs:string \\
\hline properties & use required \\
\hline source & <xs:attribute name="Name" type="xs:string" use="required"/> \\
\hline
\end{tabular}

attribute AbstractApplicationType/@Start

\begin{tabular}{|r|l|}
\hline type & xs:double \\
\hline properties & $\begin{array}{c}\text { use optional } \\
\text { default } 1.0\end{array}$ \\
\hline source & <xs:attribute name="Start" type="xs:double" use="optional" default="1.0"/> \\
\hline
\end{tabular}

attribute AbstractApplicationType/@Stop

\begin{tabular}{|r|l|}
\hline type & xs:double \\
\hline properties & $\begin{array}{c}\text { use optional } \\
\text { default } 10.0\end{array}$ \\
\hline source & <xs:attribute name="Stop" type="xs:double" use="optional" default="10.0"/> \\
\hline
\end{tabular}


complexType PromiscuousModeType

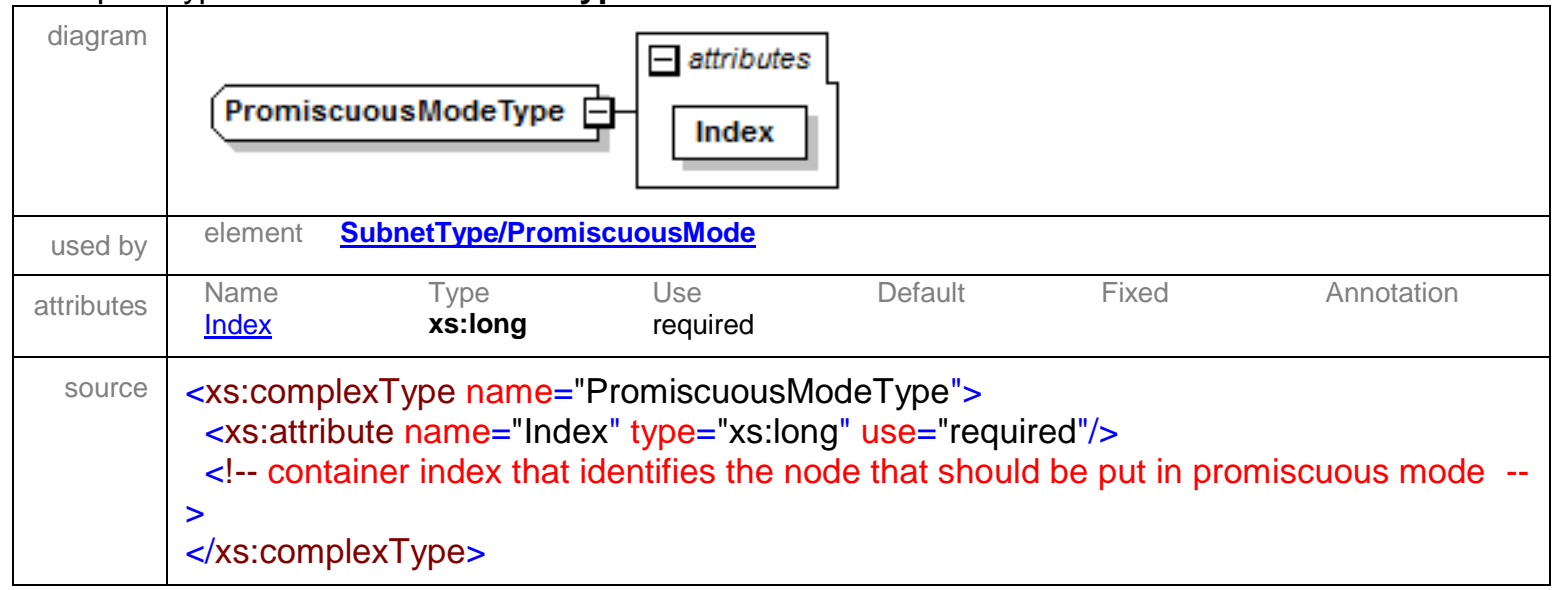

attribute PromiscuousModeType/@Index

\begin{tabular}{|c|c|}
\hline type & xs:long \\
\hline properties & use required \\
\hline source & <xs:attribute name="Index" type="xs:long" use="required"/> \\
\hline
\end{tabular}

simpleType HostTypeEnum

\begin{tabular}{|c|c|}
\hline type & restriction of xs:string \\
\hline properties & base xs:string \\
\hline used by & attribute $\quad$ RefNodelnterfaceDefinitionType/@Type \\
\hline facets & $\begin{array}{lll}\text { Kind } & \text { Value } & \text { Annotation } \\
\text { enumeration } & \text { SIMPLE } & \\
\text { enumeration } & \text { SWITCH } & \\
\text { enumeration } & \text { ROUTER } & \end{array}$ \\
\hline source & $\begin{array}{l}\text { <xs:simpleType name="HostTypeEnum"> } \\
\text { <xs:restriction base="xs:string"> } \\
\text { <xs:enumeration value="SIMPLE"/> } \\
\text { <xs:enumeration value="SWITCH"/> } \\
\text { <xs:enumeration value="ROUTER"/> } \\
\text { </xs:restriction> } \\
\text { </xs:simpleType> }\end{array}$ \\
\hline
\end{tabular}

simpleType IPv4AddressType

\begin{tabular}{|c|c|}
\hline type & restriction of xs:string \\
\hline properties & base xs:string \\
\hline used by & 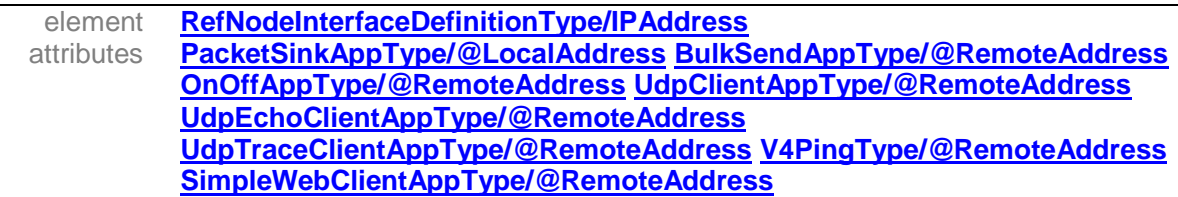 \\
\hline
\end{tabular}


LLNL-TR-630332

\begin{tabular}{|c|c|}
\hline facets & $\begin{array}{ll}\text { Kind } & \text { Value } \\
\text { pattern } & ((25[0-5]|2[0-4][0-9]| 1[0-9][0-9]|[1-9][0-9]|[0-9]) \backslash .)\{3\}(25[0-5]|2[0-4][0-9]| 1[0-9][0- \\
& 9]|[1-9][0-9]|[0-9])\end{array}$ \\
\hline annotation & IPv4 address in the dotted-decimal notation. \\
\hline source & $\begin{array}{l}\text { <xs:simpleType name="IPv4AddressType"> } \\
\text { <xs:annotation> } \\
\text { <xs:documentation> } \\
\qquad \text { IPv4 address in the dotted-decimal notation. } \\
\quad</ \text { xs:documentation> } \\
\text { </xs:annotation> } \\
\text { <xs:restriction base="xs:string"> } \\
\text { <xs:pattern value="((25[0-5]|2[0-4][0-9]|1[0-9][0-9]|[1-9][0-9]|[0-9])\.)\{3\}(25[0-5]|2[0- } \\
\text { 4][0-9]|1[0-9][0-9]|[1-9][0-9]|[0-9])"/> } \\
\text { </xs:restriction> } \\
\text { </xs:simpleType> }\end{array}$ \\
\hline
\end{tabular}

simpleType PercentType

\begin{tabular}{|c|c|}
\hline type & restriction of xs:long \\
\hline properties & base xs:long \\
\hline used by & attributes AssignAppSetType/@Percent ApplicationSetType/@Percent \\
\hline facets & $\begin{array}{lll}\text { Kind } & \text { Value } & \text { Annotation } \\
\text { minlnclusive } & 0 & \\
\text { maxlnclusive } & 100 & \end{array}$ \\
\hline source & $\begin{array}{l}\text { <xs:simpleType name="PercentType"> } \\
\text { <xs:restriction base="xs:long"> } \\
\text { <xs:minlnclusive value="0"/> } \\
\text { <xs:maxInclusive value="100"/> } \\
\text { </xs:restriction> } \\
\text { </xs:simpleType> }\end{array}$ \\
\hline
\end{tabular}

simpleType SubnetTypeEnum

\begin{tabular}{|c|c|}
\hline type & restriction of xs:string \\
\hline properties & base xs:string \\
\hline used by & attribute SubnetType/@Type \\
\hline facets & $\begin{array}{lll}\text { Kind } & \text { Value } & \text { Annotation } \\
\text { enumeration } & \text { CSMA } & \\
\text { enumeration } & \text { P2P } & \\
\end{array}$ \\
\hline source & $\begin{array}{l}\text { <xs:simpleType name="SubnetTypeEnum"> } \\
\text { <xs:restriction base="xs:string"> } \\
\text { <xs:enumeration value="CSMA"/> } \\
\text { <xs:enumeration value="P2P"/> } \\
\text { </xs:restriction> } \\
\text { </xs:simpleType> }\end{array}$ \\
\hline
\end{tabular}


LLNL-TR-630332

complexType BulkSendAppType

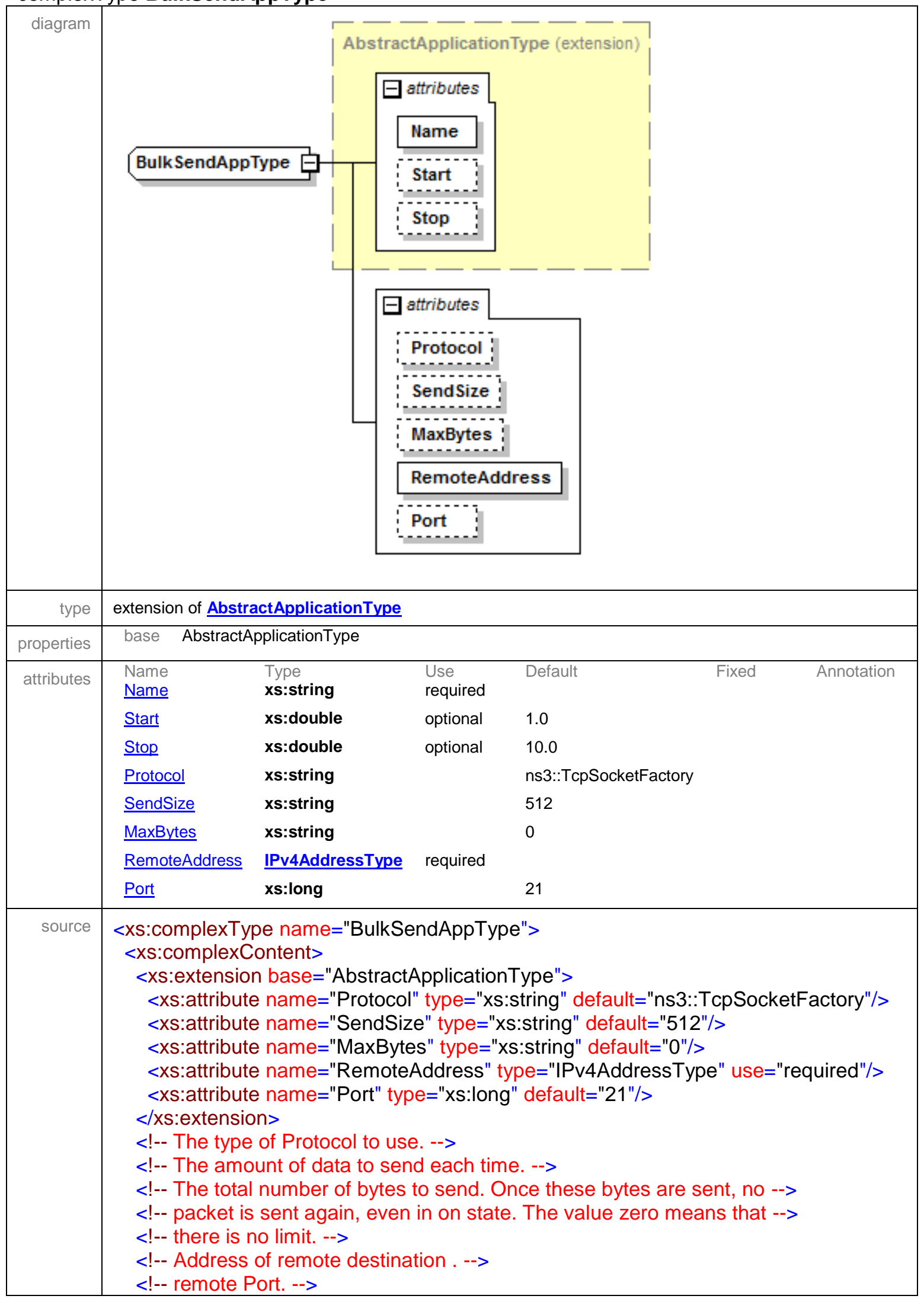


LLNL-TR-630332

$</ x s$ :complexContent $>$ $</ x s:$ complexType $>$

attribute BulkSendAppType/@Protocol

\begin{tabular}{|r|l|}
\hline type & xs:string \\
\hline properties & default ns3::TcpSocketFactory \\
\hline source & $<x$ :attribute name="Protocol" type="xs:string" default="ns3::TcpSocketFactory"/> \\
\hline
\end{tabular}

attribute BulkSendAppType/@SendSize

\begin{tabular}{|r|l|}
\hline type & xs:string \\
\hline properties & default 512 \\
\hline source & $<x$ s:attribute name="SendSize" type="xs:string" default="512"/> \\
\hline
\end{tabular}

attribute BulkSendAppType/@MaxBytes

\begin{tabular}{|r|l|}
\hline type & xs:string \\
\hline properties & default 0 \\
\hline source & $<x$ :attribute name="MaxBytes" type="xs:string" default="0"/> \\
\hline
\end{tabular}

attribute BulkSendAppType/@RemoteAddress

\begin{tabular}{|c|c|}
\hline type & IPv4AddressType \\
\hline properties & use required \\
\hline facets & $\begin{array}{ll}\text { Kind } & \text { Value } \\
\text { pattern } & ((25[0-5]|2[0-4][0-9]| 1[0-9][0-9]|[1-9][0-9]|[0-9]) \backslash .)\{3\}(25[0-5]|2[0-4][0-9]| 1[0-9][0- \\
& 9]|[1-9][0-9]|[0-9]) \\
\end{array}$ \\
\hline source & <xs:attribute name="RemoteAddress" type="IPv4AddressType" use="required"/> \\
\hline
\end{tabular}

attribute BulkSendAppType/@Port

\begin{tabular}{|r|c|}
\hline type & xs:Iong \\
\hline properties & default 21 \\
\hline source & $<x$ :attribute name="Port" type="xs:long" default="21"/> \\
\hline
\end{tabular}


LLNL-TR-630332

complexType OnOffAppType

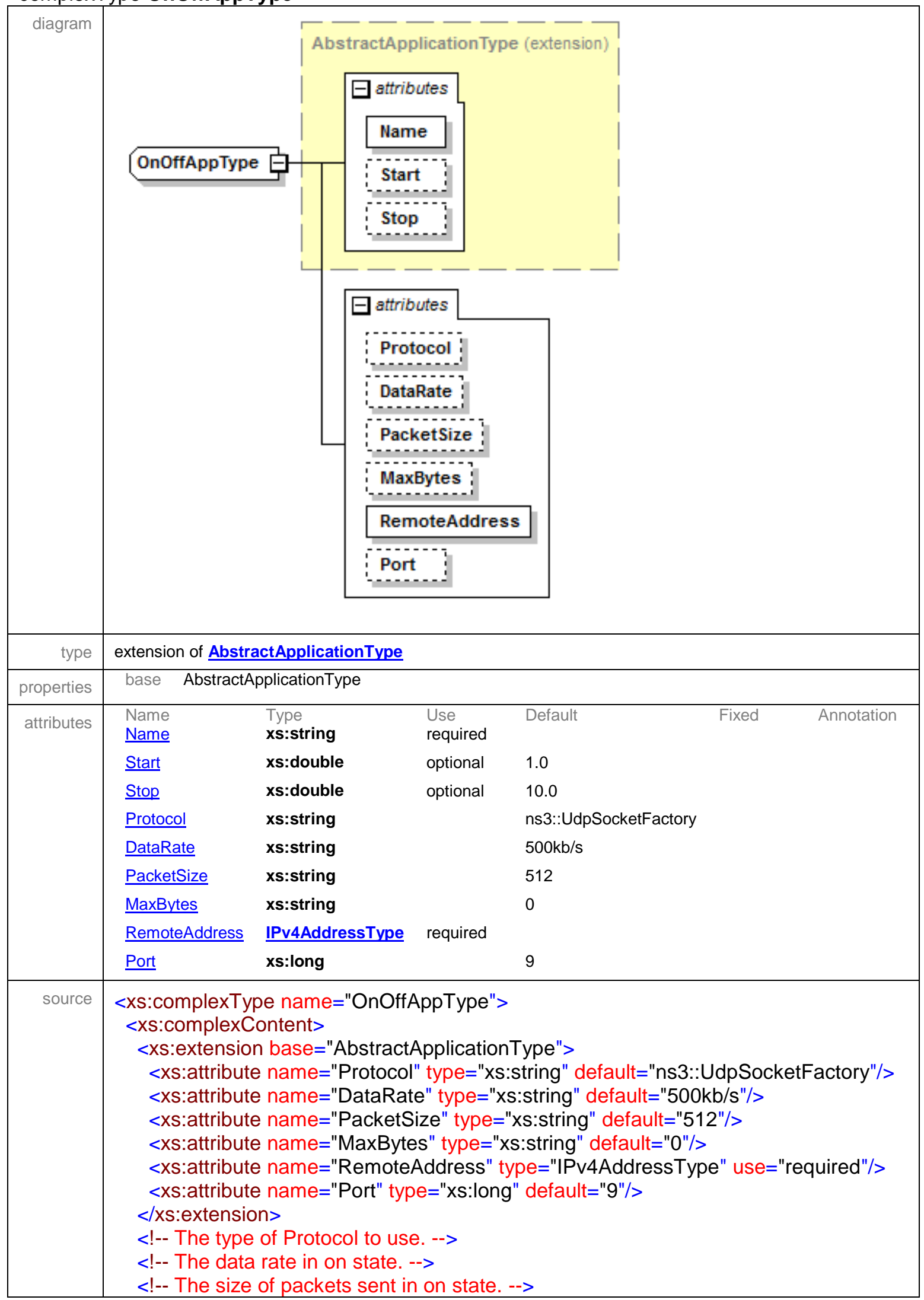




\begin{tabular}{|c|c|}
\hline & $<!--$ The total number of bytes to send. Once these bytes are sent, no --> \\
& $<!--$ packet is sent again, even in on state. The value zero means that --> \\
& $<!--$ there is no limit. --> \\
& $<!--$ Address of remote destination . --> \\
& $<!--$ remote Port. --> \\
$</ x$ :complexContent $>$ & $</$ s:complexType $>$
\end{tabular}

attribute OnOffAppType/@Protocol

\begin{tabular}{|r|l|}
\hline type & xs:string \\
\hline properties & default ns3::UdpSocketFactory \\
\hline source & <xs:attribute name="Protocol" type="xs:string" default="ns3::UdpSocketFactory"/> \\
\hline
\end{tabular}

attribute OnOffAppType/@DataRate

\begin{tabular}{|r|l|}
\hline type & xs:string \\
\hline properties & default $500 \mathrm{~kb} / \mathrm{s}$ \\
\hline source & $<x s: a t t r i b u t e ~ n a m e=" D a t a R a t e " ~ t y p e=" x s: s t r i n g " ~ d e f a u l t=" 500 \mathrm{~kb} / \mathrm{s} " />$ \\
\hline
\end{tabular}

attribute OnOffAppType/@PacketSize

\begin{tabular}{|r|l|}
\hline type & xs:string \\
\hline properties & default 512 \\
\hline source & $<x s: a t t r i b u t e ~ n a m e=" P a c k e t S i z e " ~ t y p e=" x s: s t r i n g " ~ d e f a u l t=" 512 " />$ \\
\hline
\end{tabular}

attribute OnOffAppType/@MaxBytes

\begin{tabular}{|r|l|}
\hline type & xs:string \\
\hline properties & default 0 \\
\hline source & $<x$ :attribute name="MaxBytes" type="xs:string" default="0"/> \\
\hline
\end{tabular}

attribute OnOffAppType/@RemoteAddress

\begin{tabular}{|c|c|}
\hline type & IPv4AddressType \\
\hline properties & use required \\
\hline facets & $\begin{array}{ll}\text { Kind } & \text { Value } \\
\text { pattern } & ((25[0-5]|2[0-4][0-9]| 1[0-9][0-9]|[1-9][0-9]|[0-9]) \backslash .)\{3\}(25[0-5]|2[0-4][0-9]| 1[0-9][0- \\
& 9]|[1-9][0-9]|[0-9])\end{array}$ \\
\hline source & <xs:attribute name="RemoteAddress" type="IPv4AddressType" use="required"/> \\
\hline
\end{tabular}

attribute OnOffAppType/@Port

type $\mathbf{x s : \text { long }}$


LLNL-TR-630332

\begin{tabular}{|c|c|}
\hline properties & $\begin{array}{ll}\text { default } 9 \\
\end{array}$ \\
\hline source & <xs:attribute name="Port" type="xs:long" default="9"/> \\
\hline
\end{tabular}

complexType PacketSinkAppType

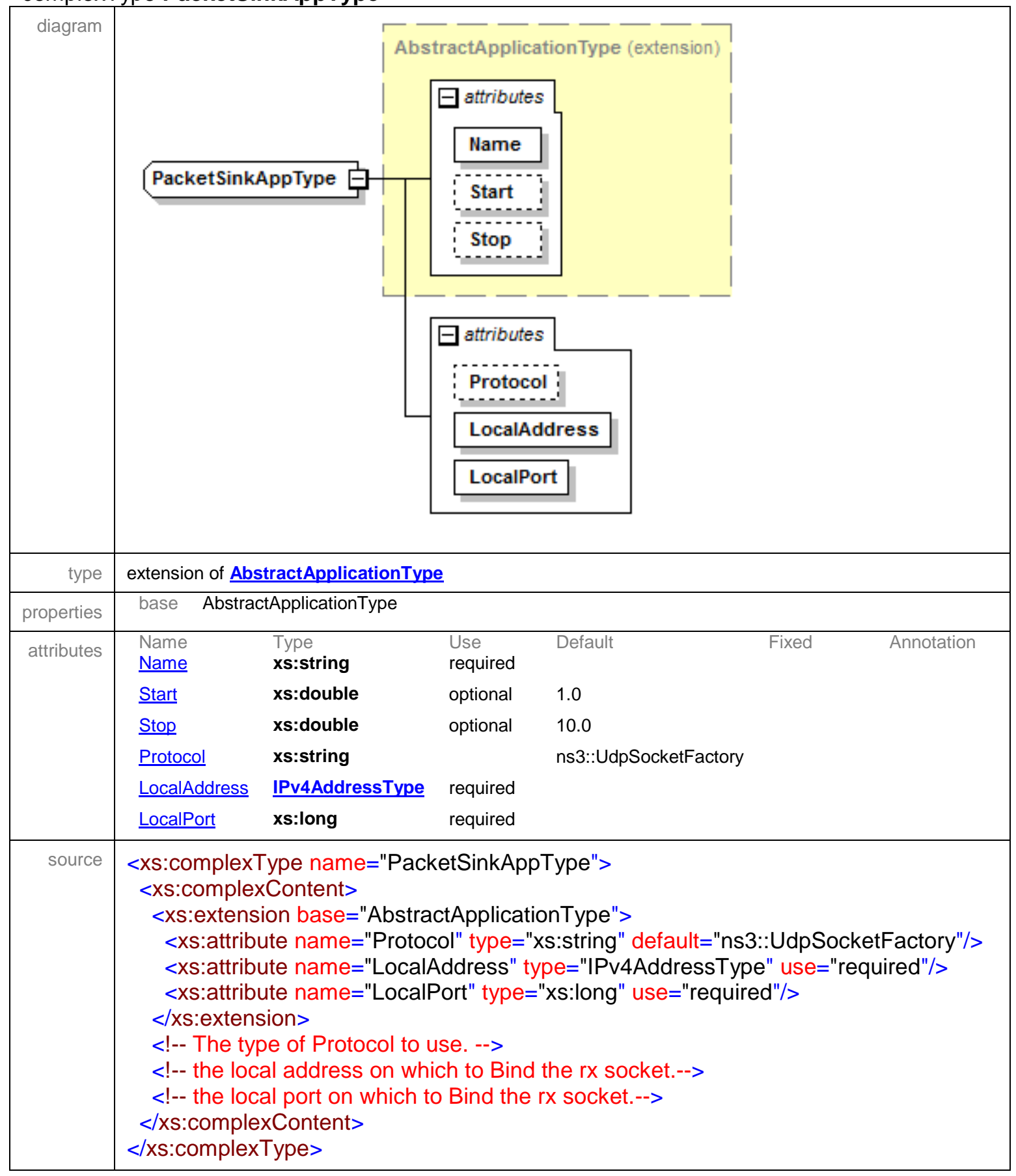


LLNL-TR-630332

attribute PacketSinkAppType/@Protocol

\begin{tabular}{|r|l|}
\hline type & xs:string \\
\hline properties & default ns3::UdpSocketFactory \\
\hline source & $<x$ :attribute name="Protocol" type="xs:string" default="ns3::UdpSocketFactory"/> \\
\hline
\end{tabular}

attribute PacketSinkAppType/@LocalAddress

\begin{tabular}{|c|c|}
\hline type & IPv4AddressType \\
\hline properties & use required \\
\hline facets & $\begin{array}{ll}\text { Kind } & \text { Value } \\
\text { pattern } & ((25[0-5]|2[0-4][0-9]| 1[0-9][0-9]|[1-9][0-9]|[0-9]) \backslash .)\{3\}(25[0-5]|2[0-4][0-9]| 1[0-9][0- \\
& 9]|[1-9][0-9]|[0-9])\end{array}$ \\
\hline source & <xs:attribute name="LocalAddress" type="IPv4AddressType" use="required"/> \\
\hline
\end{tabular}

attribute PacketSinkAppType/@LocalPort

\begin{tabular}{|c|c|}
\hline type & xs:long \\
\hline properties & use required \\
\hline source & <xs:attribute name="LocalPort" type="xs:long" use="required"/> \\
\hline
\end{tabular}

complexType UdpClientAppType

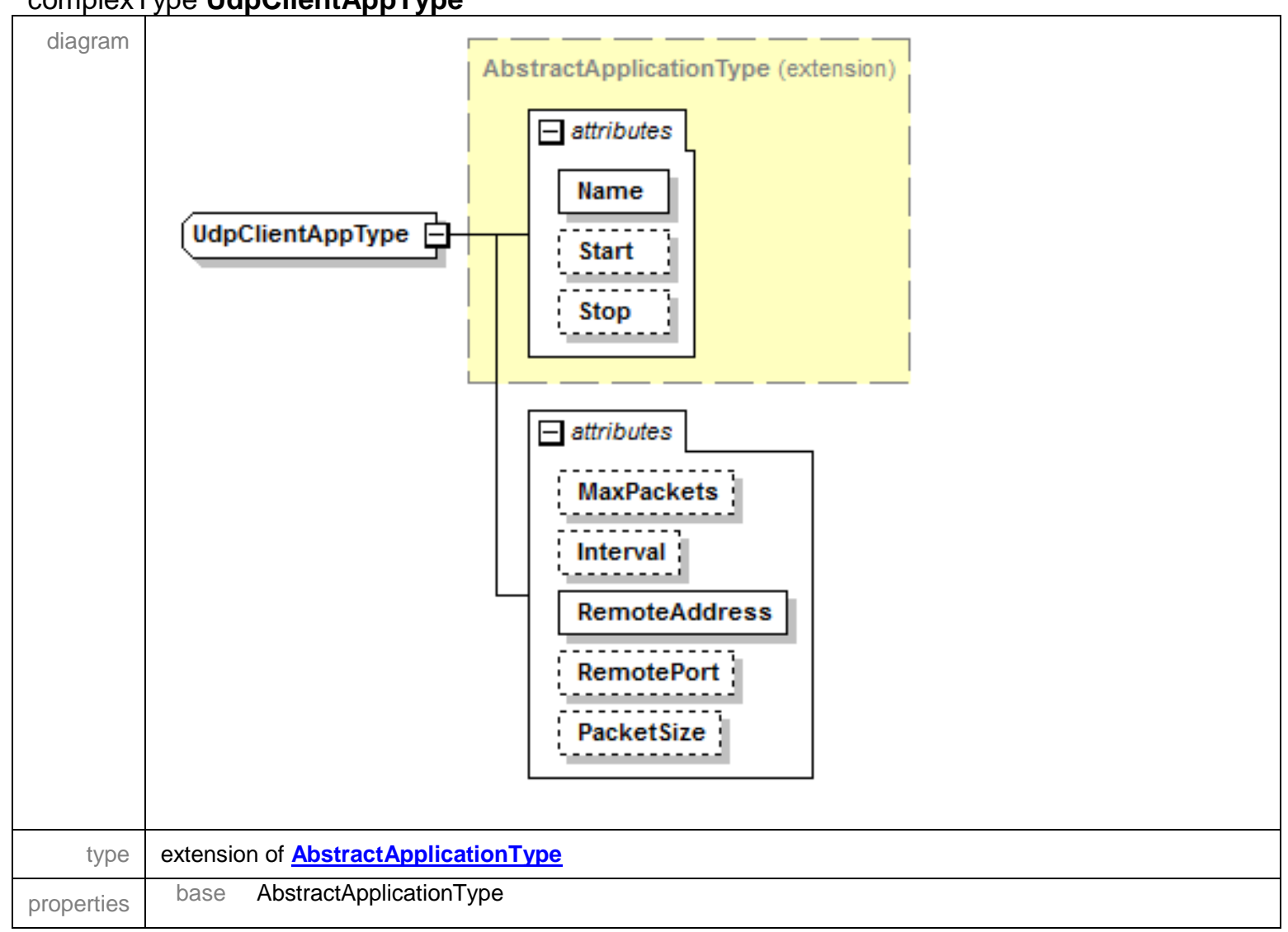


LLNL-TR-630332

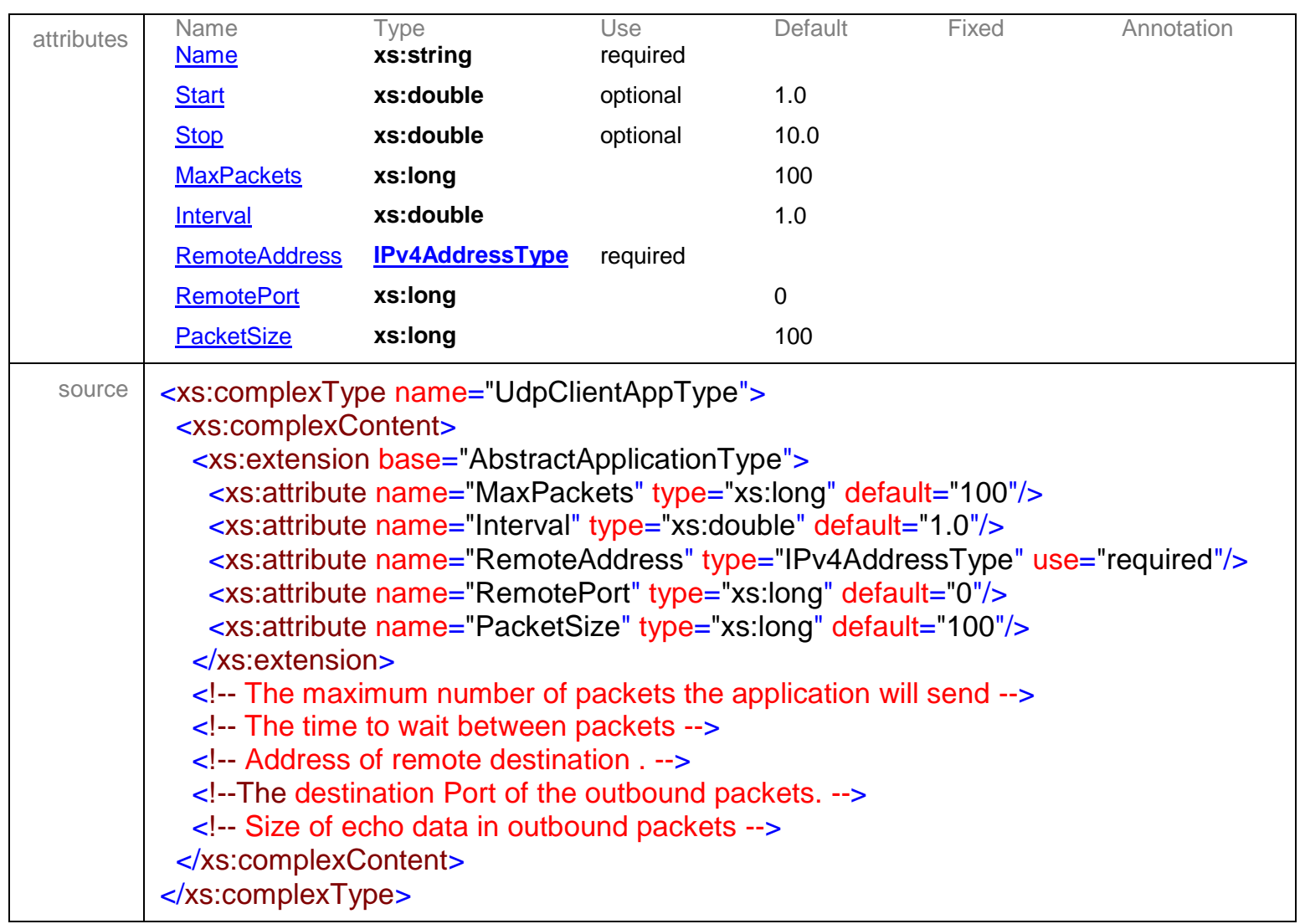

attribute UdpClientAppType/@MaxPackets

\begin{tabular}{|r|l|}
\hline type & xs:long \\
\hline properties & default 100 \\
\hline source & $<x$ s:attribute name="MaxPackets" type="xs:long" default="100"/> \\
\hline
\end{tabular}

attribute UdpClientAppType/@Interval

\begin{tabular}{|r|l|}
\hline type & xs:double \\
\hline properties & default 1.0 \\
\hline source & $<x$ :attribute name="Interval" type="xs:double" default="1.0"/> \\
\hline
\end{tabular}

attribute UdpClientAppType/@RemoteAddress

\begin{tabular}{|c|c|}
\hline type & IPv4AddressType \\
\hline properties & use required \\
\hline facets & 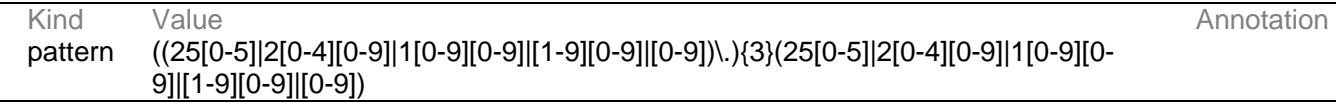 \\
\hline source & <xs:attribute name="RemoteAddress" type="IPv4AddressType" use="required"/> \\
\hline
\end{tabular}


LLNL-TR-630332

attribute UdpClientAppType/@RemotePort

\begin{tabular}{|r|l|}
\hline type & xs:long \\
\hline properties & default 0 \\
\hline source & $<x$ :attribute name="RemotePort" type="xs:long" default="0"/> \\
\hline
\end{tabular}

attribute UdpClientAppType/@PacketSize

\begin{tabular}{|r|l|}
\hline type & xs:long \\
\hline properties & default 100 \\
\hline source & $<x$ s:attribute name="PacketSize" type="xs:long" default="100"/> \\
\hline
\end{tabular}

complexType UdpServerAppType

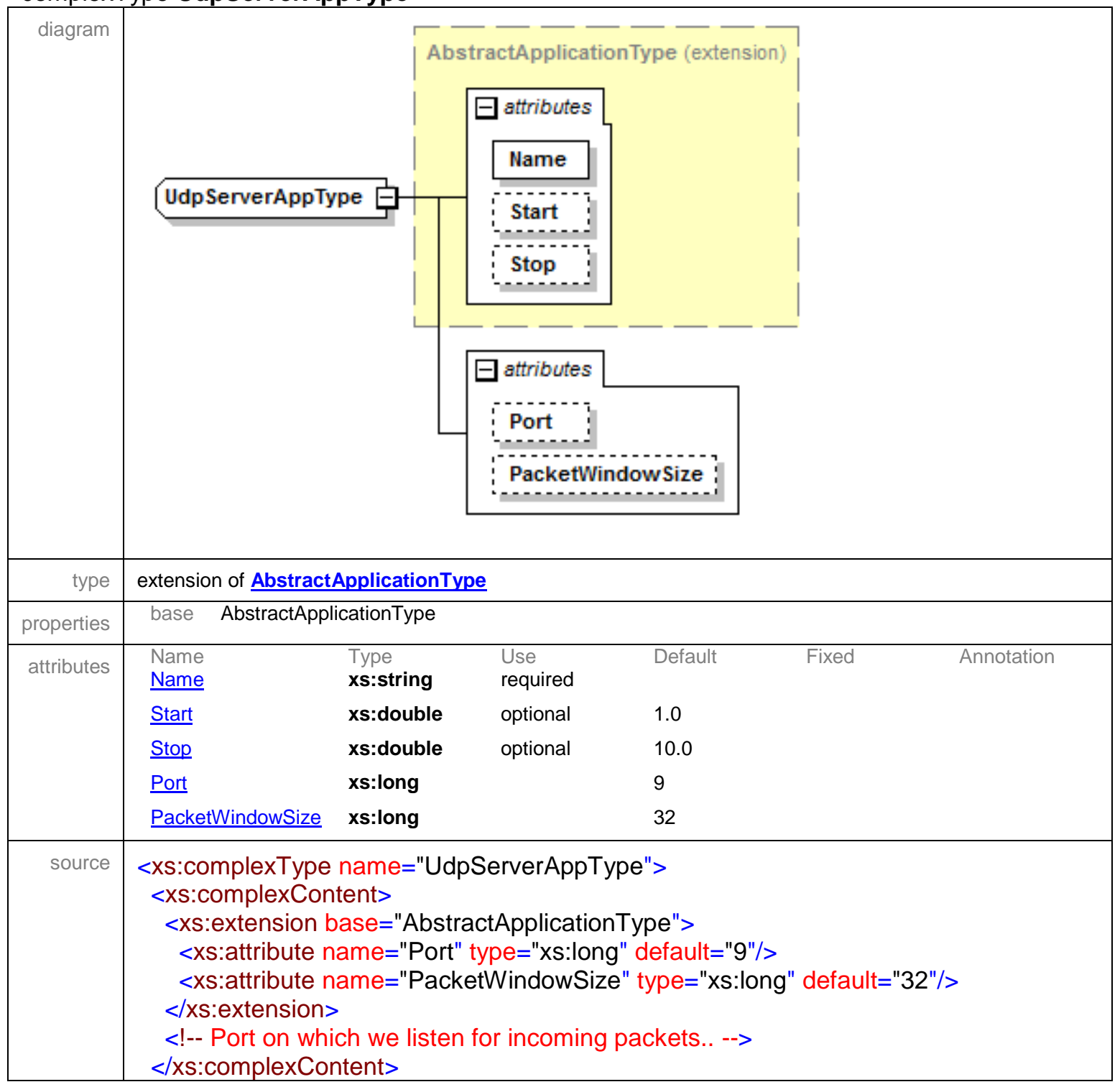


LLNL-TR-630332

$</ x s: c o m p l e x T y p e>$

attribute UdpServerAppType/@Port

\begin{tabular}{|r|l|}
\hline type & xs:long \\
\hline properties & default 9 \\
\hline source & $<x$ s:attribute name="Port" type="xs:long" default="9"/> \\
\hline
\end{tabular}

attribute UdpServerAppType/@PacketWindowSize

\begin{tabular}{|r|l|}
\hline type & xs:long \\
\hline properties & default 32 \\
\hline source & $<x$ s:attribute name="PacketWindowSize" type="xs:long" default="32"/> \\
\hline
\end{tabular}

complexType UdpEchoClientAppType

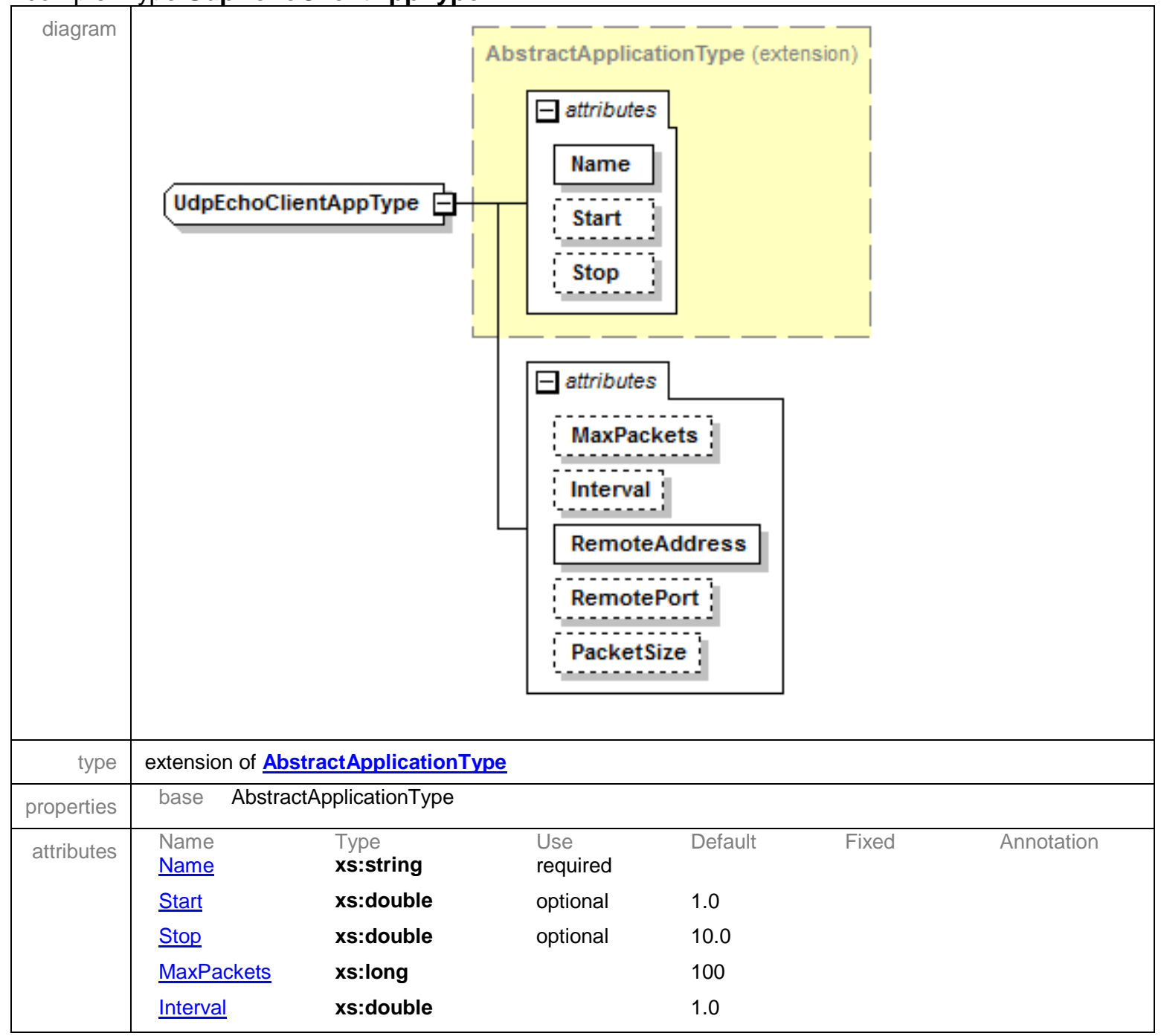


LLNL-TR-630332

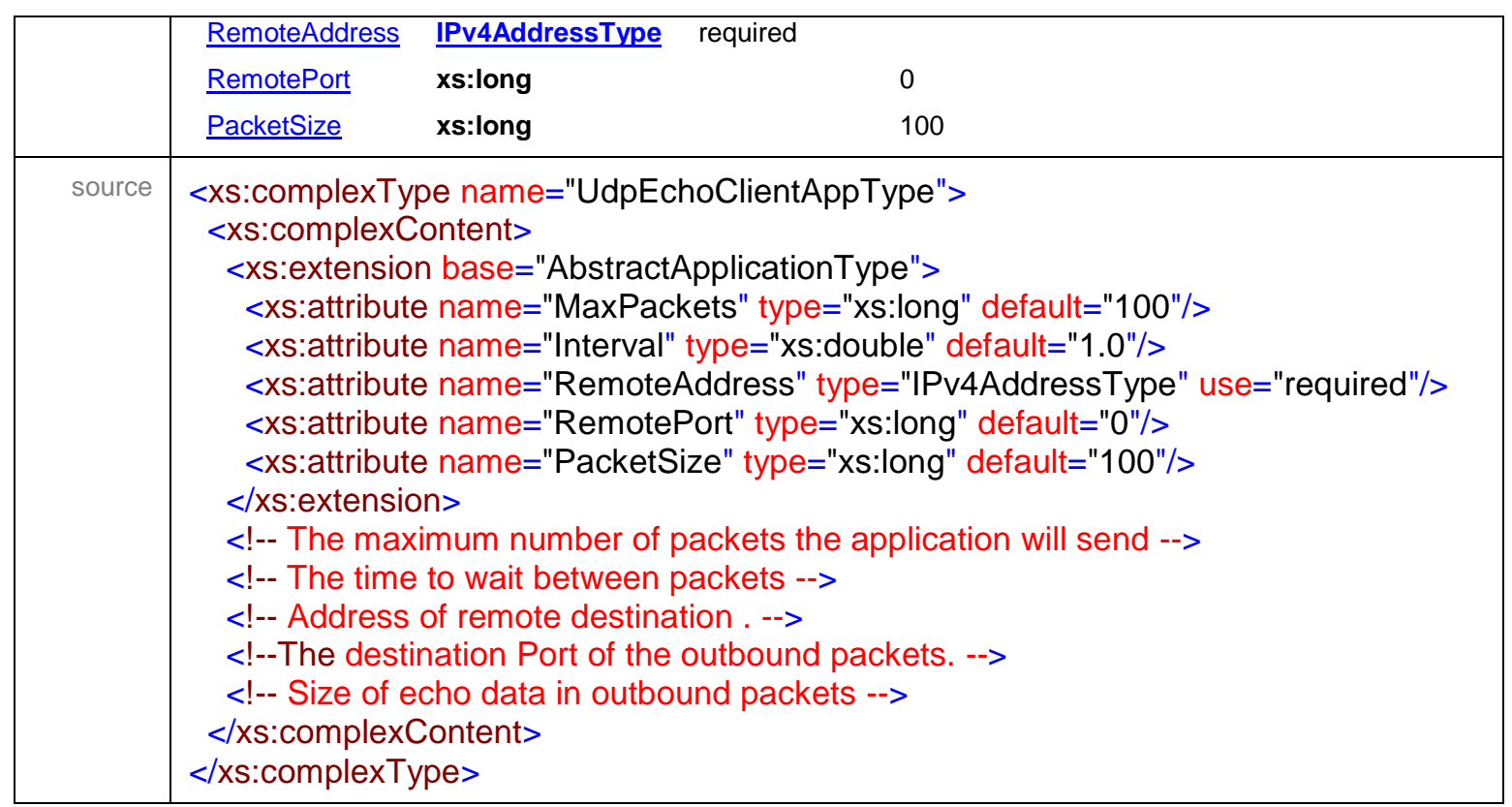

attribute UdpEchoClientAppType/@MaxPackets

\begin{tabular}{|r|l|}
\hline type & xs:long \\
\hline properties & default 100 \\
\hline source & $<x$ :attribute name="MaxPackets" type="xs:long" default="100"/> \\
\hline
\end{tabular}

attribute UdpEchoClientAppType/@Interval

\begin{tabular}{|r|l|}
\hline type & xs:double \\
\hline properties & default 1.0 \\
\hline source & $<x s:$ attribute name="Interval" type="xs:double" default="1.0"/> \\
\hline
\end{tabular}

attribute UdpEchoClientAppType/@RemoteAddress

\begin{tabular}{|r|ll|}
\hline type & IPv4AddressType \\
\hline properties & use required & Annotation \\
\hline facets & $\begin{array}{l}\text { Kind } \\
\text { pattern }\end{array}$ & $\begin{array}{l}\text { Value } \\
((25[0-5]|2[0-4][0-9]| 1[0-9][0-9]|[1-9][0-9]|[0-9]) । .)\{3\}(25[0-5]|2[0-4][0-9]| 1[0-9][0-\end{array}$ \\
\hline source & <xs:attribute name="RemoteAddress" type="IPv4AddressType" use="required"/> \\
\hline
\end{tabular}

attribute UdpEchoClientAppType/@RemotePort

\begin{tabular}{|r|c|}
\hline type & xs:Iong \\
\hline properties & default 0 \\
\hline source & <xs:attribute name="RemotePort" type="xs:long" default="0"/> \\
\hline
\end{tabular}


LLNL-TR-630332

attribute UdpEchoClientAppType/@PacketSize

\begin{tabular}{|r|l|}
\hline type & xs:long \\
\hline properties & default 100 \\
\hline source & $<x$ s:attribute name="PacketSize" type="xs:long" default="100"/> \\
\hline
\end{tabular}

complexType UdpEchoServerAppType

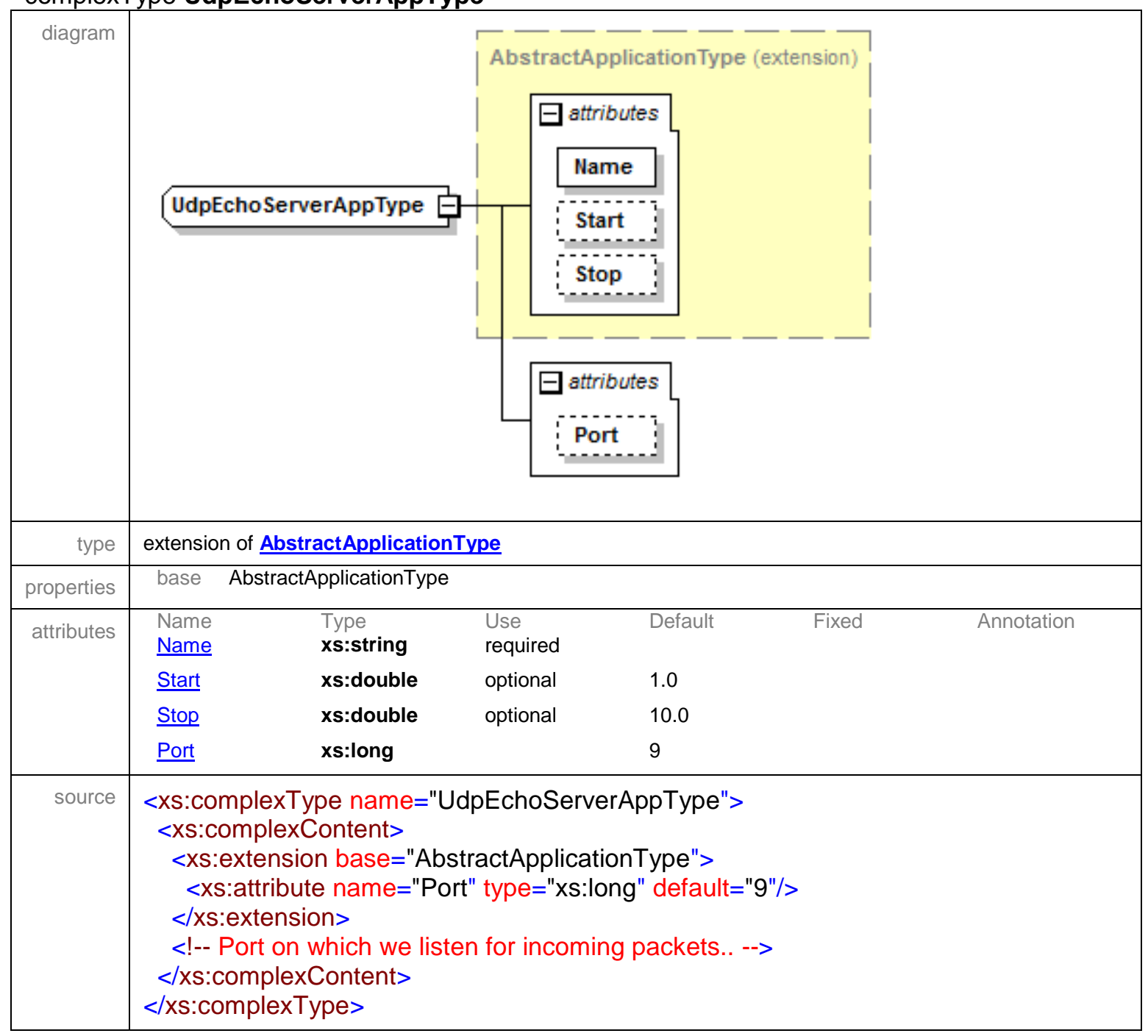

attribute UdpEchoServerAppType/@Port

\begin{tabular}{|c|c|}
\hline type & xs:long \\
\hline properties & default 9 \\
\hline source & <xs:attribute name="Port" type="xs:long" default="9"/> \\
\hline
\end{tabular}


LLNL-TR-630332

complexType UdpTraceClientAppType

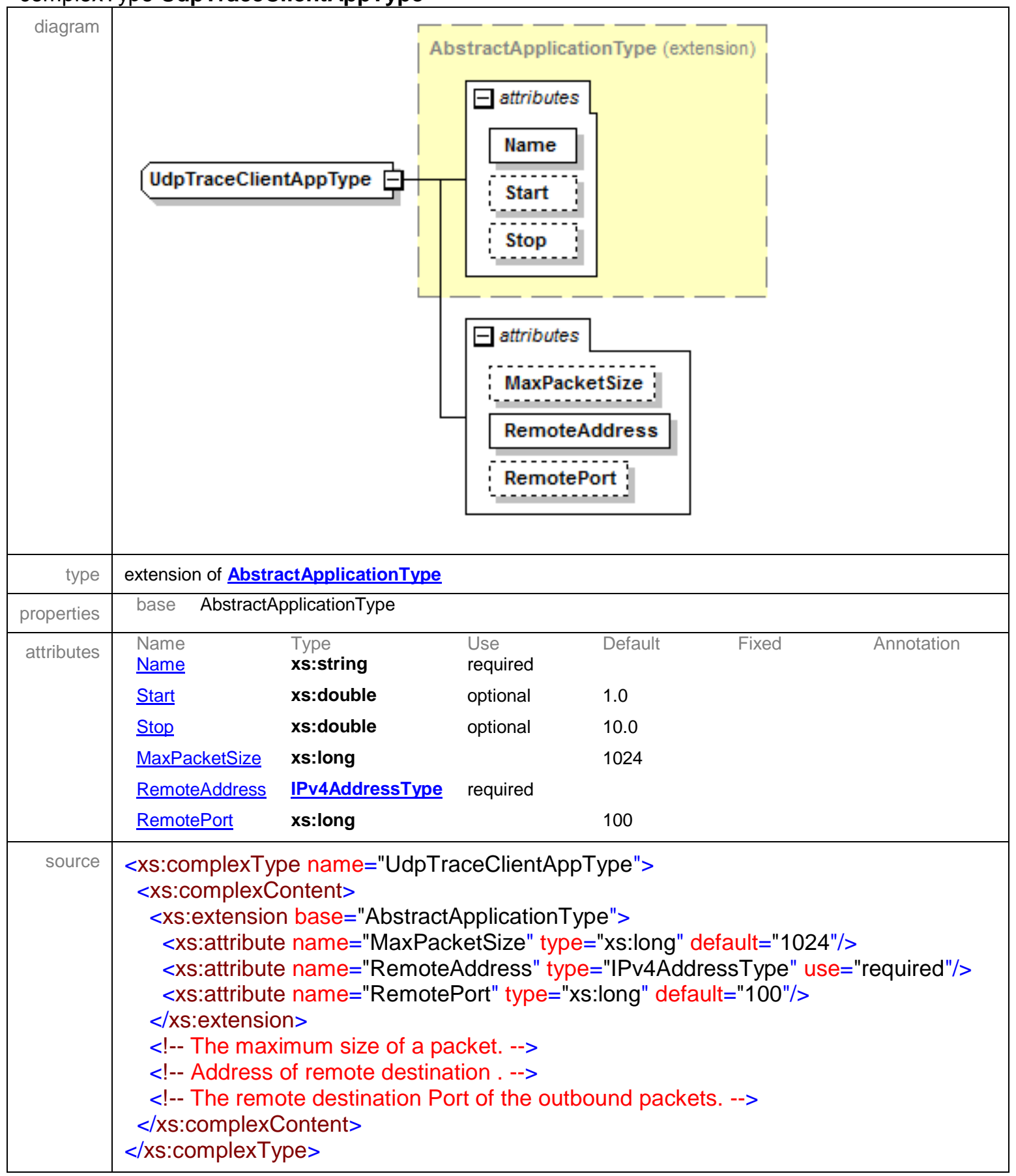

attribute UdpTraceClientAppType/@MaxPacketSize

\begin{tabular}{|r|l|}
\hline type & xs:long \\
\hline properties & default 1024 \\
\hline source & $<x$ :attribute name="MaxPacketSize" type="xs:long" default="1024"/> \\
\hline
\end{tabular}


attribute UdpTraceClientAppType/@RemoteAddress

\begin{tabular}{|c|c|}
\hline type & IPv4AddressType \\
\hline properties & use required \\
\hline facets & $\begin{array}{ll}\text { Kind } & \text { Value } \\
\text { pattern } & ((25[0-5]|2[0-4][0-9]| 1[0-9][0-9]|[1-9][0-9]|[0-9]) \backslash .)\{3\}(25[0-5]|2[0-4][0-9]| 1[0-9][0- \\
& 9]|[1-9][0-9]|[0-9])\end{array}$ \\
\hline source & <xs:attribute name="RemoteAddress" type="IPv4AddressType" use="required"/> \\
\hline
\end{tabular}

attribute UdpTraceClientAppType/@RemotePort

\begin{tabular}{|r|l|}
\hline type & xs:long \\
\hline properties & default 100 \\
\hline source & $<x$ s:attribute name="RemotePort" type="xs:long" default="100"/> \\
\hline
\end{tabular}

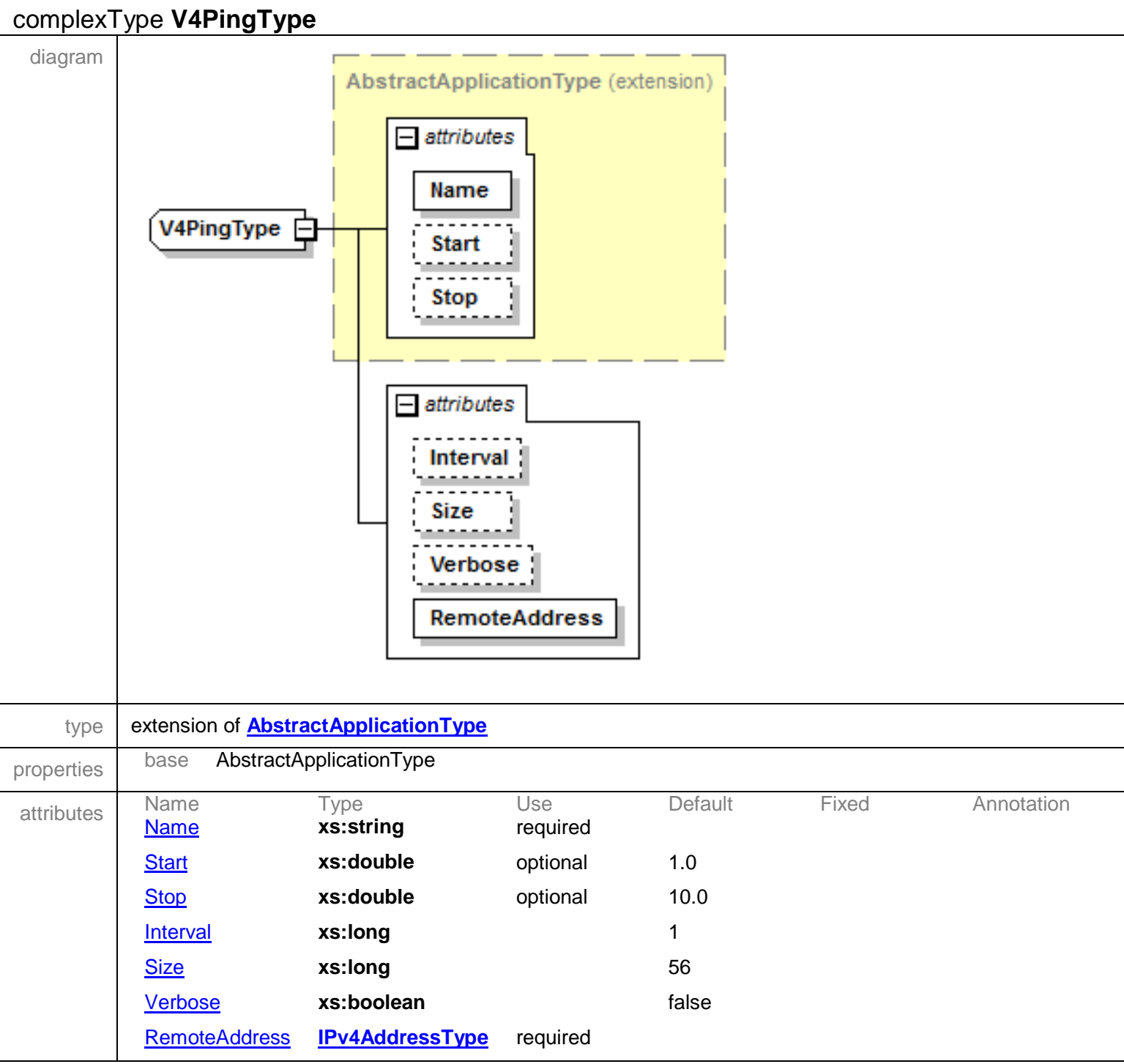




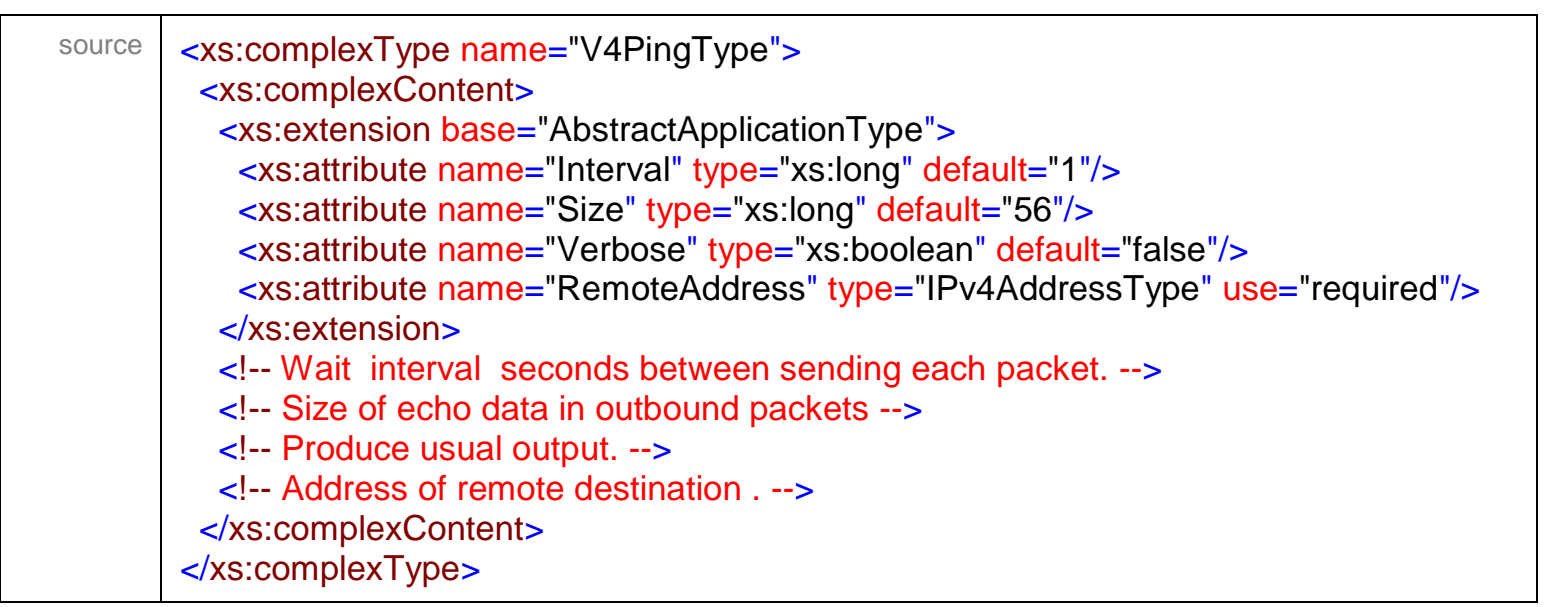

attribute V4PingType/@Interval

\begin{tabular}{|r|l|}
\hline type & xs:long \\
\hline properties & default 1 \\
\hline source & $<x$ :attribute name="Interval" type="xs:long" default="1"/> \\
\hline
\end{tabular}

attribute V4PingType/@Size

\begin{tabular}{|r|l|}
\hline type & xs:long \\
\hline properties & default 56 \\
\hline source & $<x$ s:attribute name="Size" type="xs:long" default="56"/> \\
\hline
\end{tabular}

attribute V4PingType/@Verbose

\begin{tabular}{|c|c|}
\hline type & xs:boolean \\
\hline properties & default false \\
\hline source & <xs:attribute name="Verbose" type="xs:boolean" default="false"/> \\
\hline
\end{tabular}

attribute V4PingType/@RemoteAddress

\begin{tabular}{|c|c|}
\hline type & IPv4AddressType \\
\hline properties & use required \\
\hline facets & $\begin{array}{ll}\text { Kind } & \text { Value } \\
\text { pattern } & ((25[0-5]|2[0-4][0-9]| 1[0-9][0-9]|[1-9][0-9]|[0-9]) \backslash .)\{3\}(25[0-5]|2[0-4][0-9]| 1[0-9][0- \\
& 9]|[1-9][0-9]|[0-9]) \\
\end{array}$ \\
\hline source & <xs:attribute name="RemoteAddress" type="IPv4AddressType" use="required"/> \\
\hline
\end{tabular}


complexType AttributeValuePairType

\begin{tabular}{|c|c|c|c|c|}
\hline diagram & AttributeValuePairType 而 & \begin{tabular}{|c|} 
Gattributes \\
Name \\
\hdashline Value \\
\end{tabular} & & \\
\hline used by & \multicolumn{4}{|c|}{ element GenericAppType/Attribute } \\
\hline attributes & $\begin{array}{ll}\text { Name } & \text { Type } \\
\text { Name } & \text { xs:string } \\
\text { Value } & \text { xs:string }\end{array}$ & Default & Fixed & Annotation \\
\hline source & \multicolumn{4}{|c|}{$\begin{array}{l}\text { <xs:complexType name="AttributeValuePairType"> } \\
\text { <xs:attribute name="Name" type="xs:string"/> } \\
\text { <xs:attribute name="Value" type="xs:string"/> } \\
\text { </xs:complexType> }\end{array}$} \\
\hline
\end{tabular}

attribute AttributeValuePairType/@Name

\begin{tabular}{|r|l|}
\hline type & xs:string \\
\hline source & $<x$ :attribute name="Name" type="xs:string"/> \\
\hline
\end{tabular}

attribute AttributeValuePairType/@Value

\begin{tabular}{|r|l|}
\hline type & xs:string \\
\hline source & $<x$ :attribute name="Value" type="xs:string"/> \\
\hline
\end{tabular}

complexType GenericAppType

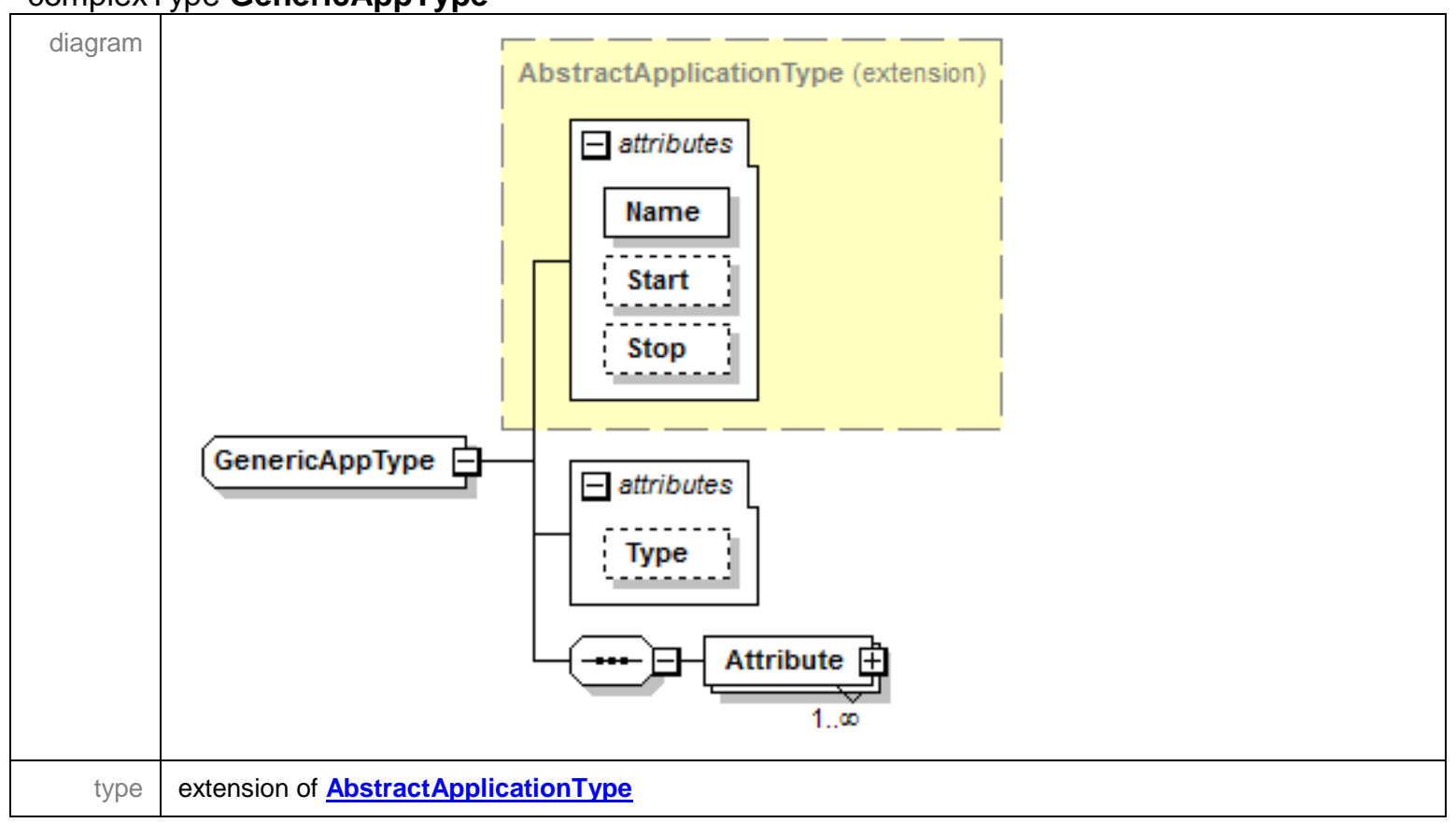


LLNL-TR-630332

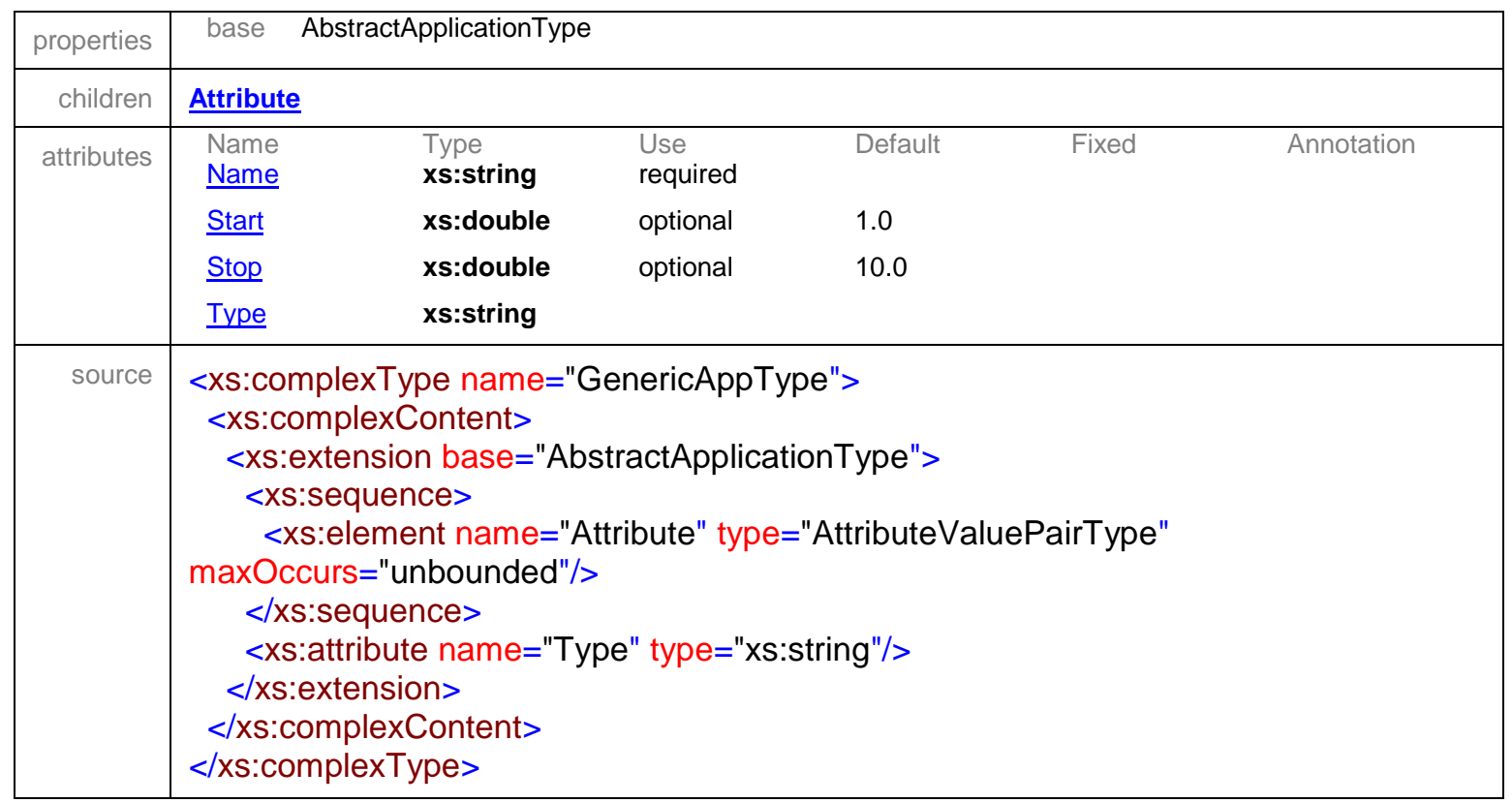

attribute GenericAppType/@Type

\begin{tabular}{|r|l|}
\hline type & xs:string \\
\hline source & $<x$ :attribute name="Type" type="xs:string"/> \\
\hline
\end{tabular}

element GenericAppType/Attribute

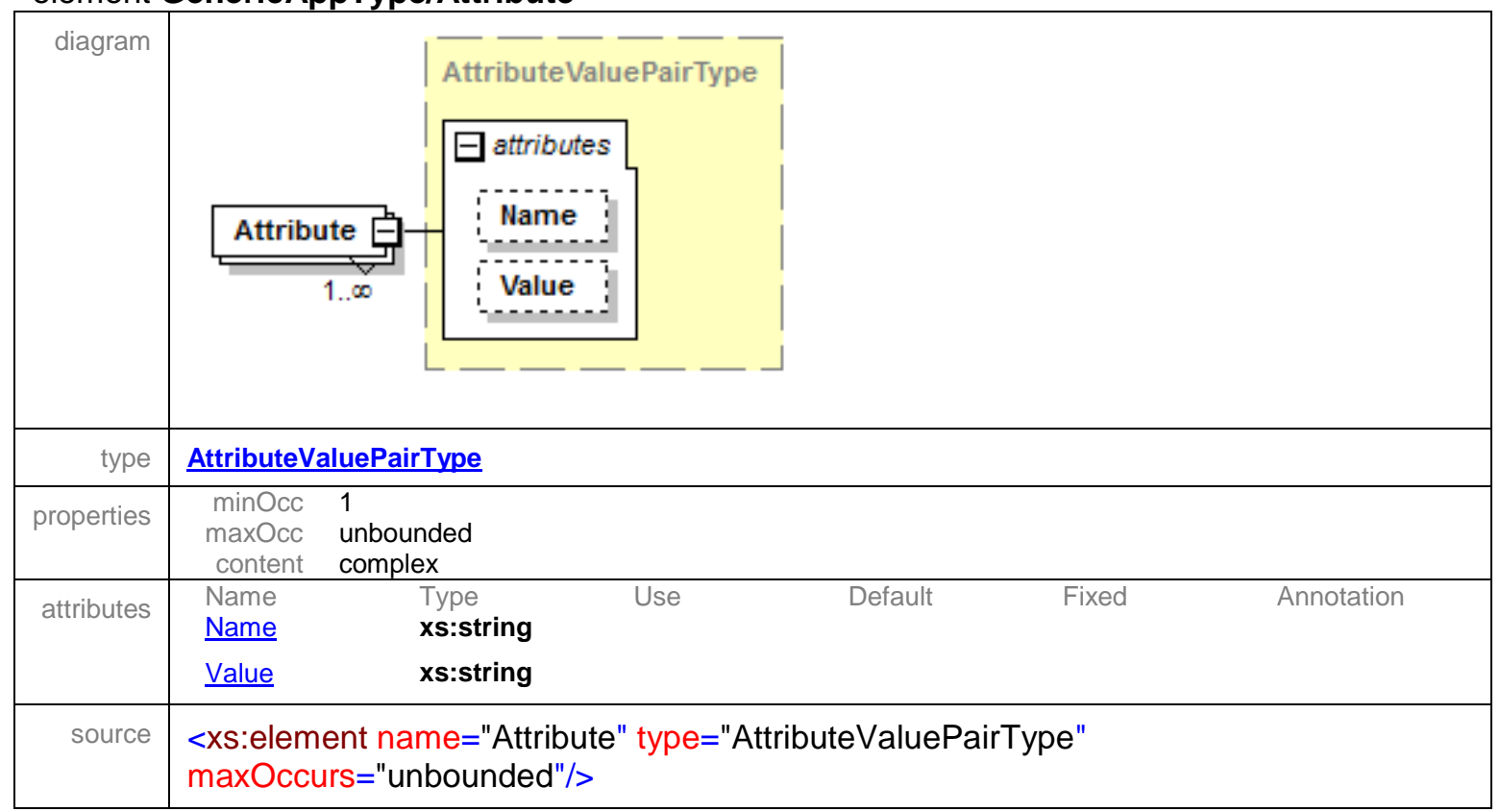


complexType SimpleWebClientAppType

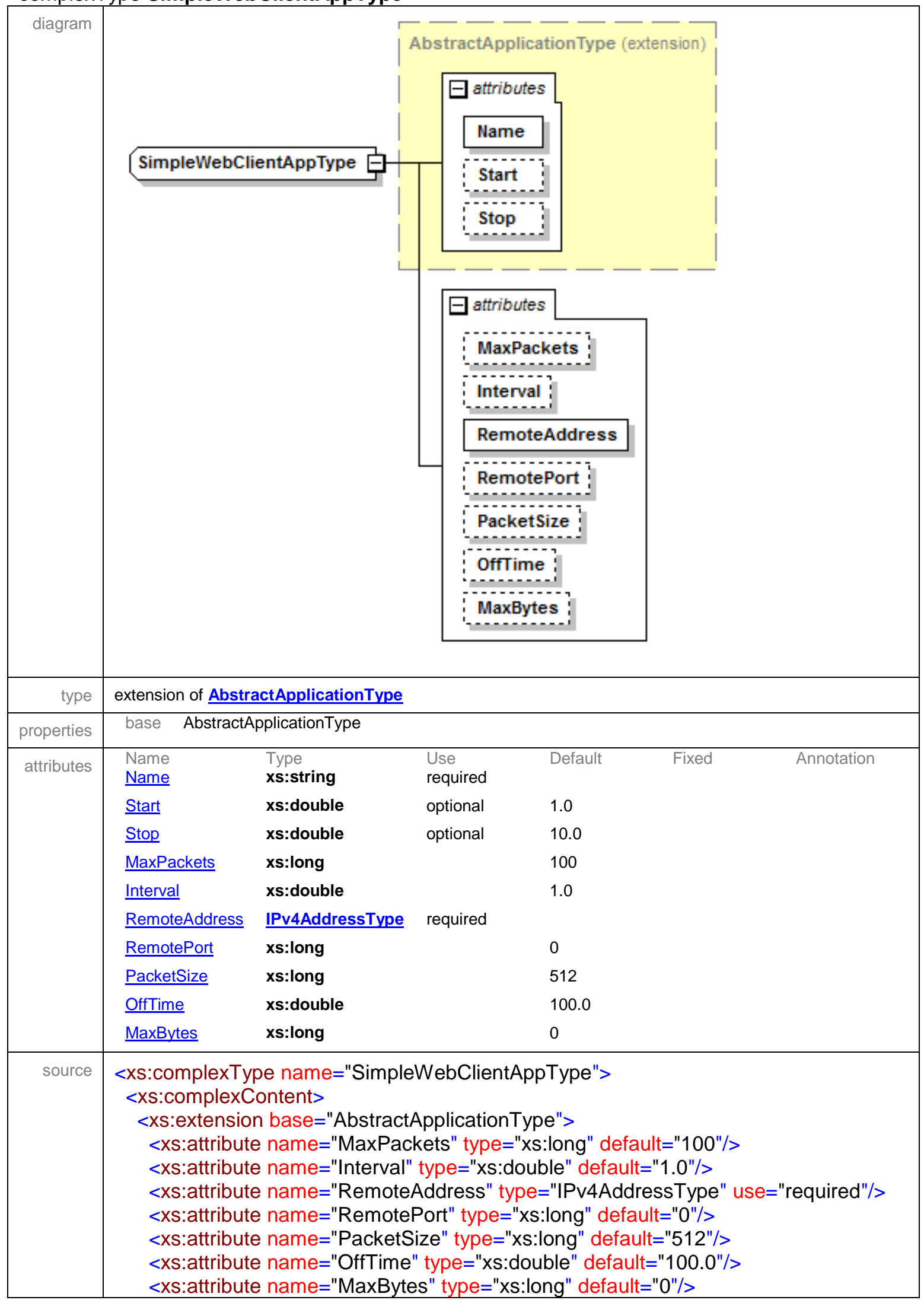




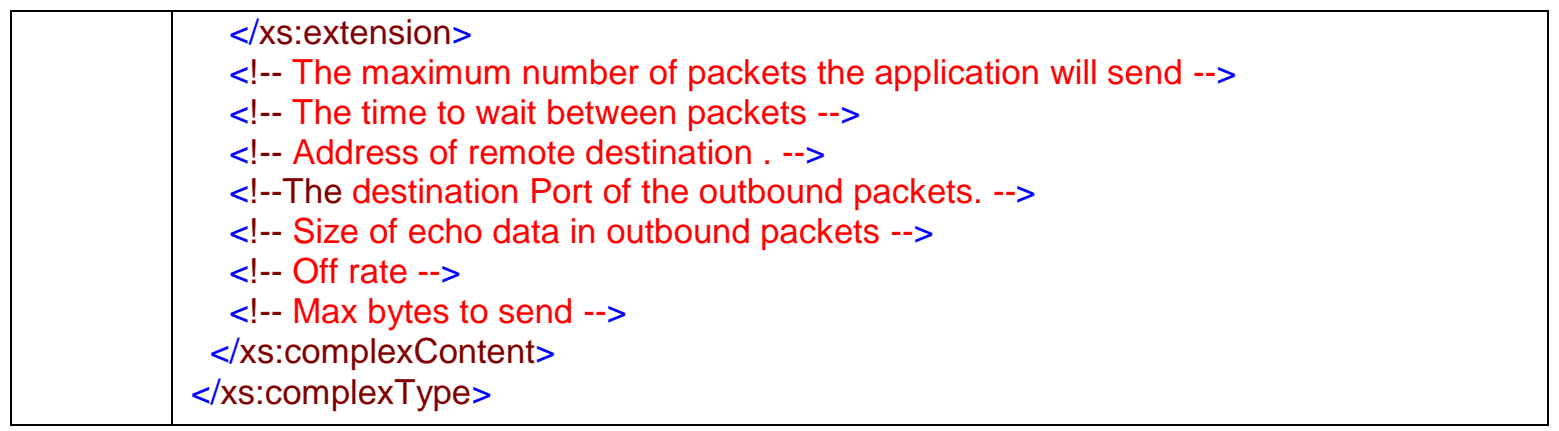

attribute SimpleWebClientAppType/@MaxPackets

\begin{tabular}{|r|l|}
\hline type & xs:long \\
\hline properties & default 100 \\
\hline source & $<x$ :attribute name="MaxPackets" type="xs:long" default="100"/> \\
\hline
\end{tabular}

attribute SimpleWebClientAppType/@Interval

\begin{tabular}{|r|l|}
\hline type & xs:double \\
\hline properties & default 1.0 \\
\hline source & $<x s:$ attribute name="Interval" type="xs:double" default="1.0"/> \\
\hline
\end{tabular}

attribute SimpleWebClientAppType/@RemoteAddress

\begin{tabular}{|c|c|}
\hline type & IPv4AddressType \\
\hline properties & use required \\
\hline facets & $\begin{array}{ll}\text { Kind } & \text { Value } \\
\text { pattern } & ((25[0-5]|2[0-4][0-9]| 1[0-9][0-9]|[1-9][0-9]|[0-9]) \backslash .)\{3\}(25[0-5]|2[0-4][0-9]| 1[0-9][0- \\
& 9]|[1-9][0-9]|[0-9]) \\
\end{array}$ \\
\hline source & <xs:attribute name="RemoteAddress" type="IPv4AddressType" use="required"/> \\
\hline
\end{tabular}

attribute SimpleWebClientAppType/@RemotePort

\begin{tabular}{|r|l|}
\hline type & xs:long \\
\hline properties & default 0 \\
\hline source & <xs:attribute name="RemotePort" type="xs:long" default="0"/> \\
\hline
\end{tabular}

attribute SimpleWebClientAppType/@PacketSize

\begin{tabular}{|r|l|}
\hline type & xs:long \\
\hline properties & default 512 \\
\hline source & $<x$ :attribute name="PacketSize" type="xs:long" default="512"/> \\
\hline
\end{tabular}


attribute SimpleWebClientAppType/@OffTime

\begin{tabular}{|r|l|}
\hline type & xs:double \\
\hline properties & default 100.0 \\
\hline source & $<x s:$ attribute name="OffTime" type="xs:double" default="100.0"/> \\
\hline
\end{tabular}

attribute SimpleWebClientAppType/@MaxBytes

\begin{tabular}{|r|l|}
\hline type & xs:long \\
\hline properties & default 0 \\
\hline source & $<x$ :attribute name="MaxBytes" type="xs:long" default="0"/> \\
\hline
\end{tabular}

complexType SimpleWebServerAppType

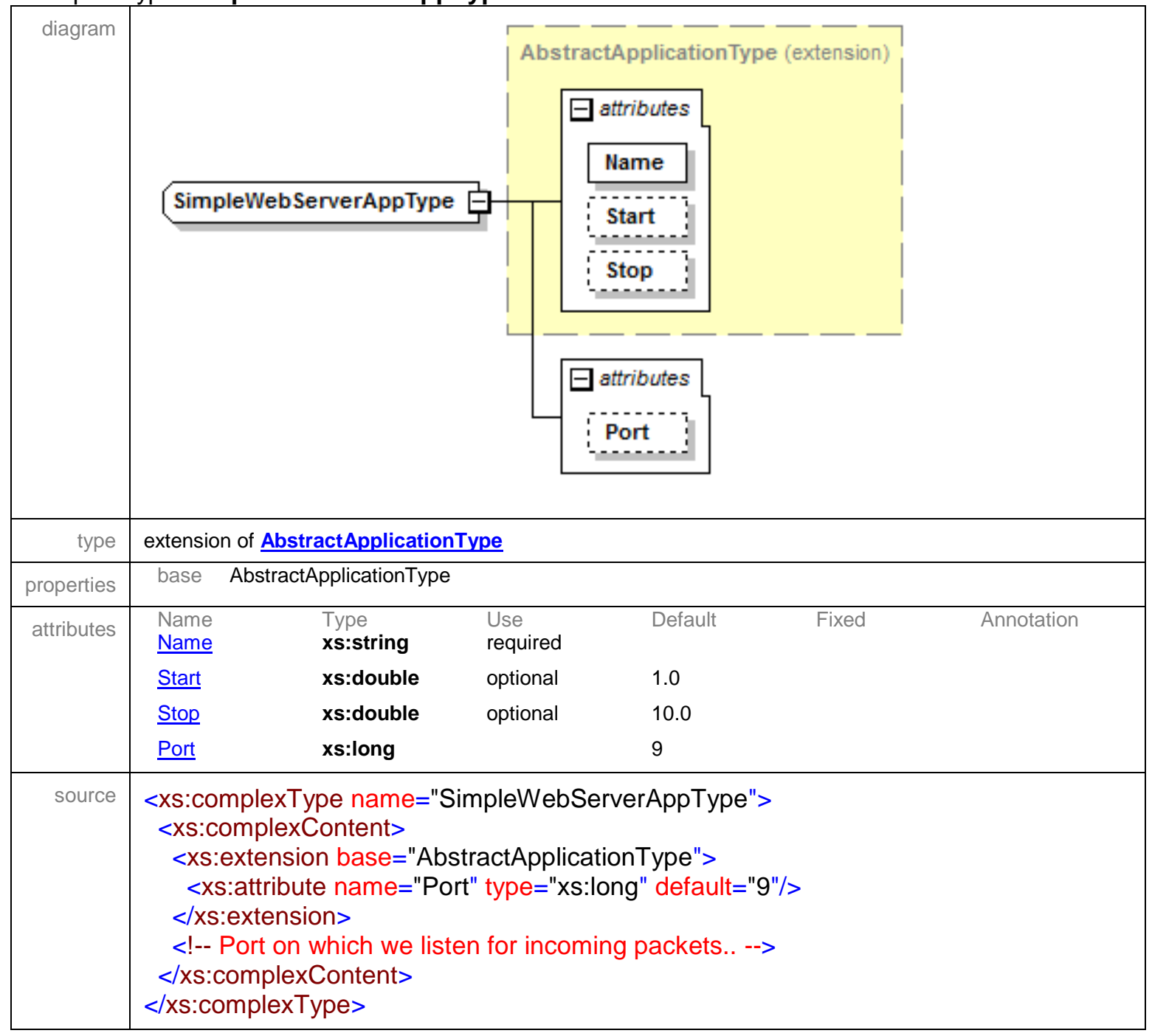


LLNL-TR-630332

attribute SimpleWebServerAppType/@Port

\begin{tabular}{|r|l|}
\hline type & xs:long \\
\hline properties & default 9 \\
\hline source & $<x$ :attribute name="Port" type="xs:long" default="9"/> \\
\hline
\end{tabular}

XML Schema documentation generated by XMLSpy Schema Editor http://www.altova.com/xmlspy 